\title{
Biomimetic Syntheses of Analogs of Hongoquercin A and B by Late-Stage Derivatization Supporting Information
}

Thomas Mies ${ }^{1}$, Andrew. J. P. White ${ }^{1}$, Philip J. Parsons ${ }^{1}$, and Anthony G. M. Barrett ${ }^{1 *}$

${ }^{1}$ Department of Chemistry, Imperial College, Molecular Sciences Research Hub, White City Campus, Wood Lane, London W12 0BZ, England.

\section{Table of Content}

\section{NMR-Data}

(E)-3,7-Dimethylocta-2,6-dien-1-yl benzoate (15)

(2E,6E)-8-Bromo-3,7-dimethylocta-2,6-dien-1-yl benzoate (17)

(2E,6E)-3,7-Dimethyl-11-(trimethylsilyl)undeca-2,6-dien-10-yn-1-ol (18)

$(2 E, 6 E)-3,7-D i m e t h y l u n d e c a-2,6-d i e n-10-y n-1-o l ~(13)$

(2E,6E)-3,7-Dimethylundeca-2,6-dien-10-yn-1-yl 4-(2,2-dimethyl-4-oxo-4H-1,3-dioxin-6-yl)-3-oxobutanoate (12)

8-((2E,6E)-3,7-Dimethylundeca-2,6-dien-10-yn-1-yl)-7-hydroxy-2,2,5-trimethyl-4H-benzo[d][1,3]dioxin-4-one (9)

( \pm )-2,2,5,7a, 13a-Pentamethyl-7a,8,9a,12,13,13a,13b,14-octahydro-4H,9H-benzo[a][1,3]dioxino[5,4-j]xanthen-4-one (8)

(E)-7-Hydroxy-2,2,5-trimethyl-8-(3-methyl-5-(2-methylcyclohexa-2,5-dien-1-yl)pent-2-en-1-yl)-4H-benzo[d][1,3]dioxin-4-one, (E)-7-hydroxy-2,2,5-trimethyl-8-(3-methyl-5-(6methylenecyclohex-2-en-1-yl)pent-2-en-1-yl)-4H-benzo[d][1,3]dioxin-4-one, and (E)-7-hydroxy-2,2,5-trimethyl-8-(3-methyl-5-(2-methylcyclohexa-1,5-dien-1-yl)pent-2-en-1-yl)$4 H$-benzo[d][1,3]dioxin-4-one (21)

( \pm )-2,2,5,8-tetramethyl-8-(4-methyloct-3-en-7-yn-1-yl)-9,10-dihydro-4H,8H-[1,3]dioxino[4,5-f]chromen-4-one (27)

( \pm )-2,2,5,7a, 13a-Pentamethyl-7a,8,9a, 10,11,12,13,13a,13b,14-decahydro-4H,9H-benzo[a][1,3]dioxino[5,4-j]xanthen-4-one (29)

( \pm -11-Hydroxy-6a,9,12b-trimethyl-1,3,4,4a,5,6,6a,12,12a,12b-decahydro-2H-benzo[a]xanthene-10-carboxylic acid (30)

( \pm -11-Hydroxy-6a,9,12b-trimethyl-1,4a,5,6,6a,12,12a,12b-octahydro-2H-benzo[a]xanthene-10-carboxylic acid (31)

( \pm )-3a,6,9,9,11b-Pentamethyl-1a,1b,3,3a, 11,11a,11b,12,13,13a-decahydro-2H,7H-[1,3]dioxino[4,5-a]oxireno[2',3':5,6]benzo[1,2-j]xanthen-7-one (32)

( \pm -11-Hydroxy-10-iodo-2,2,5,7a,13a-pentamethyl-7a,8,9a,10,11,12,13,13a,13b,14-decahydro-4H,9H-benzo[a][1,3]dioxino[5,4-j]xanthen-4-one (33)

( \pm -11-Hydroxy-2,2,5,7a, 13a-pentamethyl-7a,8,9a, 10,11,12,13,13a,13b,14-decahydro-4H,9H-benzo[a][1,3]dioxino[5,4-j]xanthen-4-one (34)

( \pm )-3,11-Dihydroxy-6a,9,12b-trimethyl-1,3,4,4a,5,6,6a,12,12a,12b-decahydro-2H-benzo[a]xanthene-10-carboxylic acid (35)

( \pm )-6a, 9,12b-Trimethyl-1,3,4,4a,5,6,6a, 12,12a,12b-decahydro-2H-benzo[a]xanthene-3,11-diol (36)

( \pm )-2,2,5,7a, 13a-Pentamethyl-7a,8,9a, 12,13,13a,13b,14-octahydro-4H,9H-benzo[a][1,3]dioxino[5,4-j]xanthene-4,11(10H)-dione (37)

( \pm )-11-Hydroxy-2,2,5,7a,13a-pentamethyl-7a,8,9a, 10,11,12,13,13a, 13b,14-decahydro-4H,9H-benzo[a][1,3]dioxino[5,4-]xxanthen-4-one (38)

( \pm )-3,11-Dihydroxy-6a,9,12b-trimethyl-1,3,4,4a,5,6,6a,12,12a,12b-decahydro-2H-benzo[a]xanthene-10-carboxylic acid (39)

( \pm )-13-Hydroxy-6a,10,10,14b-tetramethyl-1,4,4a,5,6,6a,9,14,14a,14b-decahydro- $2 \mathrm{H}, 10 \mathrm{H}$-benzo[a]pyrano[4,3-i]xanthene-3,12-dione (40)

( \pm )-7-(hydroxymethyl)-3a,6,9b-trimethyl-1a, 1b,3,3a,9,9a,9b,10,11,11a-decahydro-2H-oxireno[2',3':3,4]benzo[1,2-a]xanthen-8-ol (41)

( \pm )-10-Bromo-11-hydroxy-2,2,5,7a,13a-pentamethyl-7a,8,9a,10,11,12,13,13a,13b,14-decahydro-4H,9H-benzo[a][1,3]dioxino[5,4-]xxanthen-4-one (42)

( \pm )-11-Hydroxy-2,2,5,7a, 10,13a-hexamethyl-7a,8,9a,10,11,12,13,13a,13b, 14-decahydro-4H,9H-benzo[a][1,3]dioxino[5,4-J]xanthen-4-one (43) 
( \pm )-3,11-dihydroxy-4,6a,9,12b-tetramethyl-1,3,4,4a,5,6,6a,12,12a,12b-decahydro-2H-benzo[a]xanthene-10-carboxylic acid (44)

( \pm )-2,2,5,7a,10,13a-Hexamethyl-7a,8,9a,12,13,13a,13b,14-octahydro-4H,9H-benzo[a][1,3]dioxino[5,4-J]xanthene-4,11(10H)-dione (45)

( \pm )-11-Hydroxy-2,2,5,7a, 10,13a-hexamethyl-7a,8,9a,10,11,12,13,13a,13b,14-decahydro-4H,9H-benzo[a][1,3]dioxino[5,4-j]xanthen-4-one (46)

( \pm )-3,11-dihydroxy-4,6a,9,12b-tetramethyl-1,3,4,4a,5,6,6a,12,12a,12b-decahydro-2H-benzo[a]xanthene-10-carboxylic acid (47)

( \pm -13-Hydroxy-4,6a, 10,10,14b-pentamethyl-1,4,4a,5,6,6a,9,14,14a,14b-decahydro-2H,10H-benzo[a]pyrano[4,3-1]xanthene-3,12-dione (48)

( \pm )-10-Azido-11-hydroxy-2,2,5,7a,13a-pentamethyl-7a,8,9a,10,11,12,13,13a,13b,14-decahydro-4H,9H-benzo[a][1,3]dioxino[5,4-]xxanthen-4-one (49)

( \pm )-10-Azido-2,2,5,7a,13a-pentamethyl-4-oxo-7a,8,9a,10,11,12,13,13a,13b,14-decahydro-4H,9H-benzo[a][1,3]dioxino[5,4-J]xanthen-11-yl acetate (50)

( \pm )-10-Amino-2,2,5,7a, 13a-pentamethyl-4-oxo-7a,8,9a, 10,11,12,13,13a,13b,14-decahydro-4H,9H-benzo[a][1,3]dioxino[5,4-]xanthen-11-yl acetate (51)

( \pm )-10-Acetamido-2,2,5,7a,13a-pentamethyl-4-oxo-7a,8,9a, 10,11,12,13,13a,13b,14-decahydro-4H,9H-benzo[a][1,3]dioxino[5,4-j]xanthen-11-yl acetate (52)

\section{Crystallography-Data}

X-ray crystallography data of 8,33 42-A, 42-B and 45

The crystal structure of 8 ( $50 \%$ probability ellipsoids)

The crystal structure of $\mathbf{3 3}$ (50\% probability ellipsoids)

The structure of 42-A, one of the two independent molecules present in the crystal of $\mathbf{4 2}$ (50\% probability ellipsoids)

The structure of $\mathbf{4 2 - B}$, one of the two independent molecules present in the crystal of $\mathbf{4 2}$ (50\% probability ellipsoids)

The crystal structure of $\mathbf{4 5}$ (50\% probability ellipsoids) 
${ }^{1} \mathrm{H}-\mathrm{NMR}\left(400 \mathrm{MHz}, \mathrm{CDCl}_{3}\right) \delta 8.08-8.03(\mathrm{~m}, 2 \mathrm{H}), 7.58-7.53(\mathrm{~m}, 1 \mathrm{H}), 7.43(\mathrm{dd}$, $J=8.4,7.0 \mathrm{~Hz}, 2 \mathrm{H}), 5.47(\mathrm{tp}, J=7.0,1.3 \mathrm{~Hz}, 1 \mathrm{H}), 5.09(\mathrm{ddq}, J=8.3,5.6,1.5 \mathrm{~Hz}$,

1H), $4.84(\mathrm{dq}, J=7.1,0.7 \mathrm{~Hz}, 2 \mathrm{H}), 2.19-2.10(\mathrm{~m}, 2 \mathrm{H}), 2.10-2.04(\mathrm{~m}, 2 \mathrm{H}), 1.77$ (d, $J=1.7 \mathrm{~Hz}, 3 \mathrm{H}), 1.67(\mathrm{~d}, J=1.3 \mathrm{~Hz}, 3 \mathrm{H}), 1.61(\mathrm{~d}, J=1.3 \mathrm{~Hz}, 3 \mathrm{H})$.

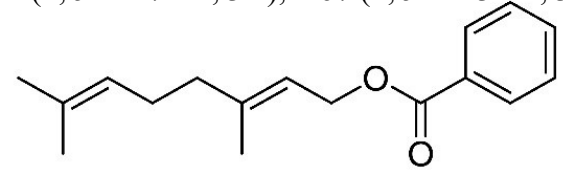

(15)
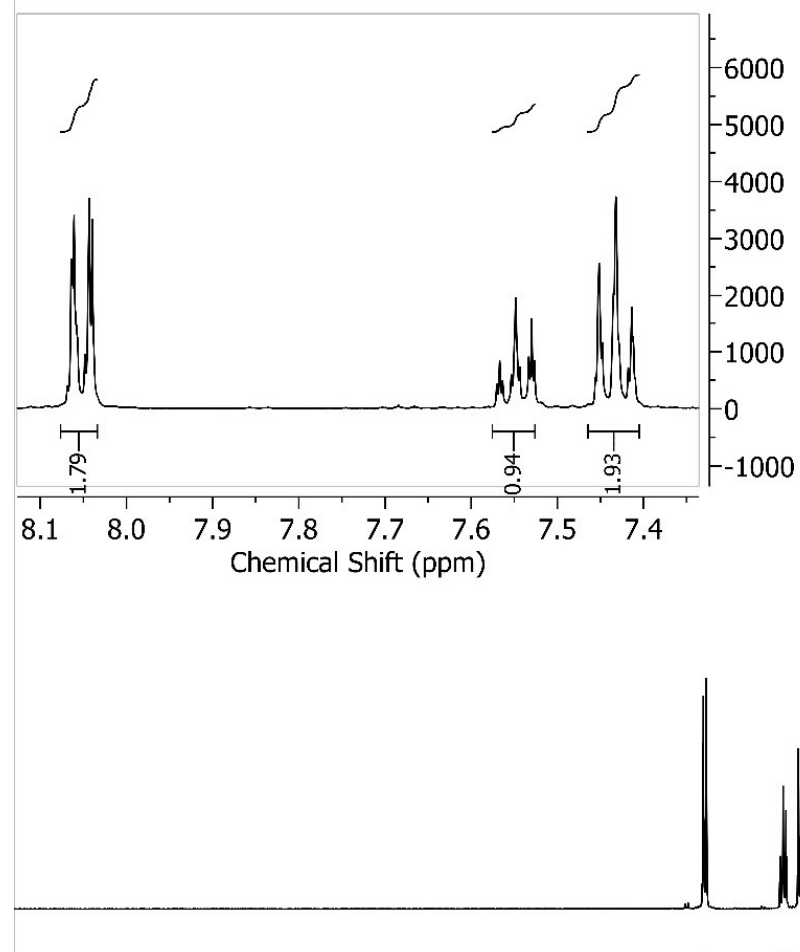
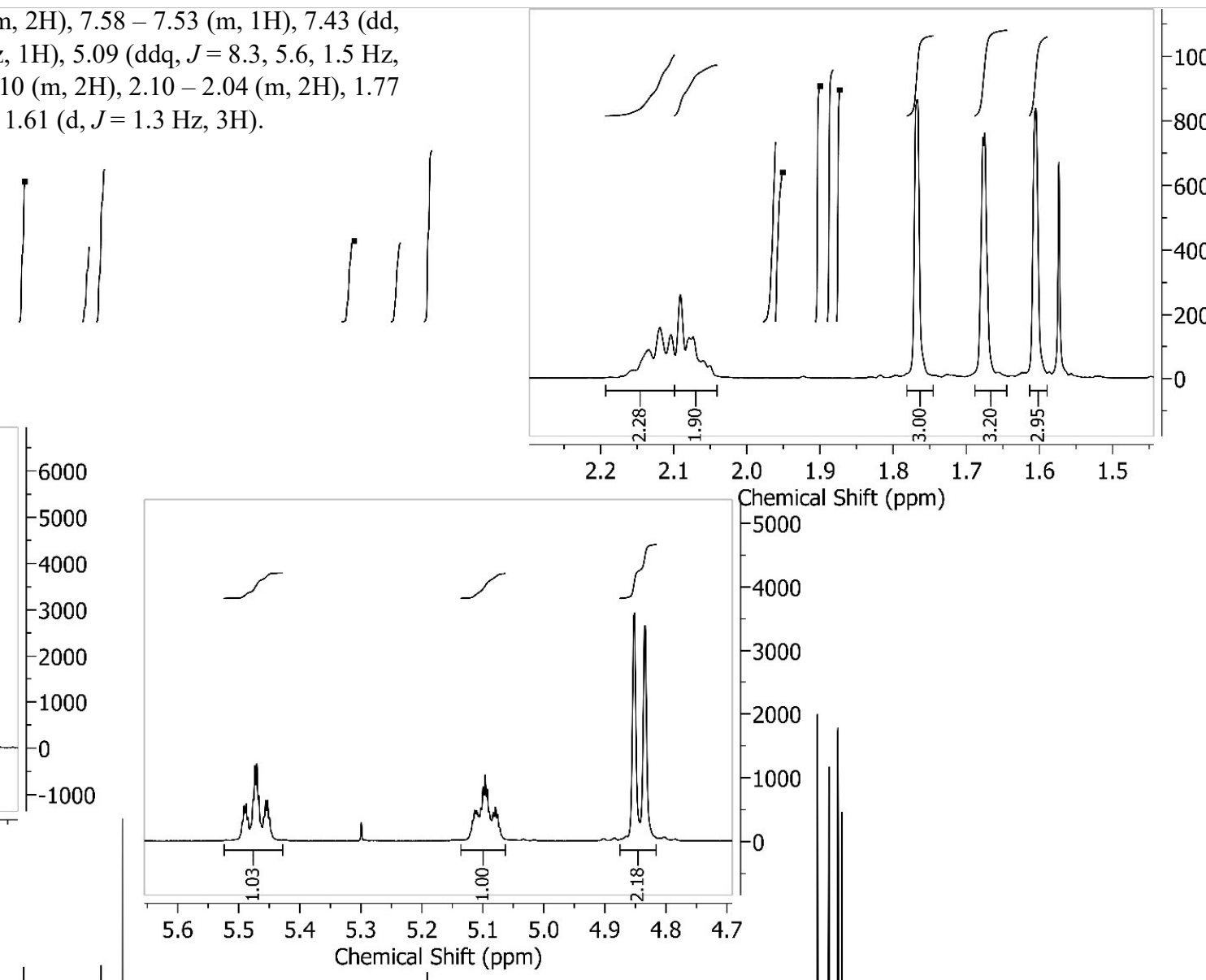

Chemical Shift (ppm)

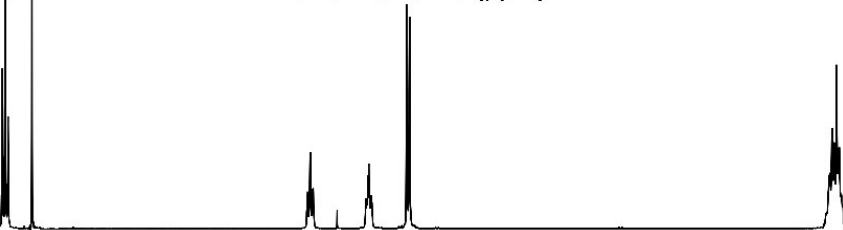

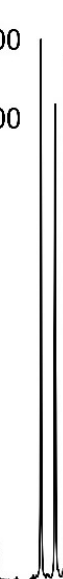

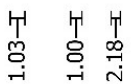

管
$-22000$ 21000 20000 $-19000$ $-18000$ $-17000$ $-16000$ $-15000$ $-14000$ 13000 12000 $-11000$ 10000 $-9000$ $-8000$ 7000 $-6000$ $-5000$ $-4000$ $-3000$ $-2000$ $-1000$ 0 $-2000$

Figure S1: ${ }^{1} \mathrm{H}-\mathrm{NMR}$ spectrum of (E)-3,7-Dimethylocta-2,6-dien-1-yl benzoate (15). 


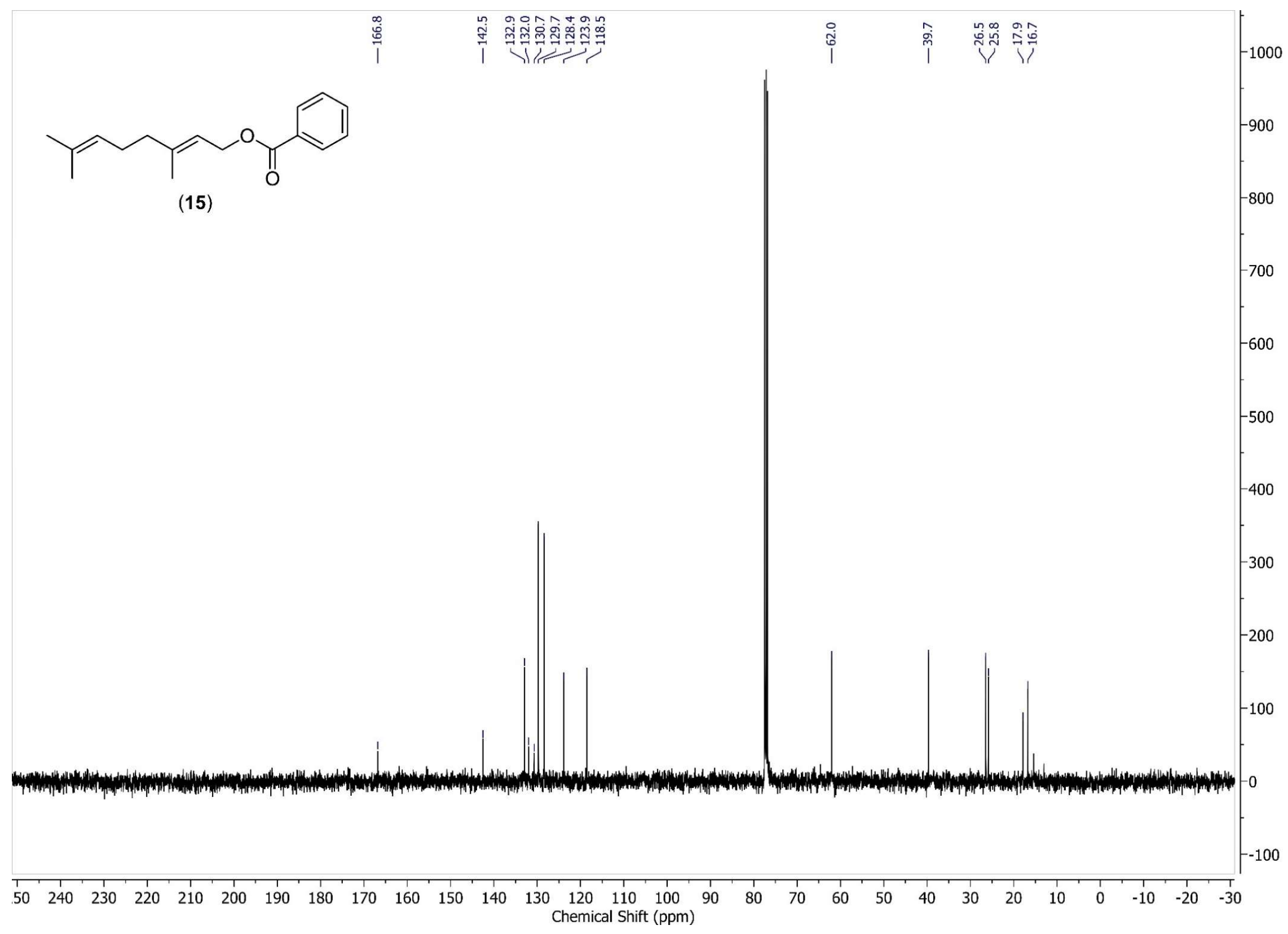

Figure S2: ${ }^{13} \mathrm{C}\left\{{ }^{1} \mathrm{H}\right\}-\mathrm{NMR}\left(101 \mathrm{MHz}, \mathrm{CDCl}_{3}\right)$ spectrum of $(E)-3,7-$ Dimethylocta-2,6-dien-1-yl benzoate (15). 


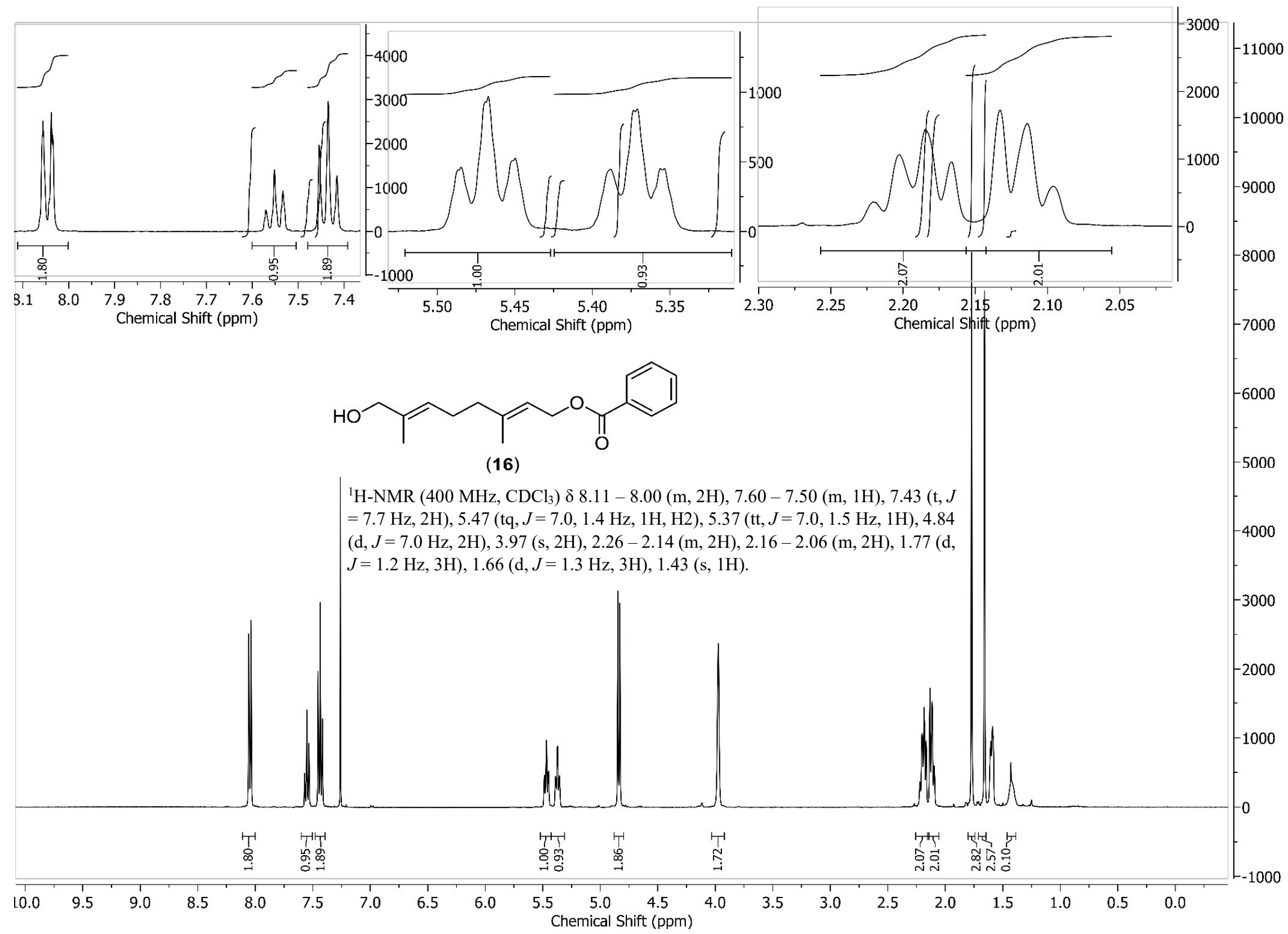

Figure S3: ${ }^{1} \mathrm{H}-\mathrm{NMR}$ spectrum of $(2 E, 6 E)-8$-Hydroxy-3,7-dimethylocta-2,6-dien-1-yl benzoate (16). 


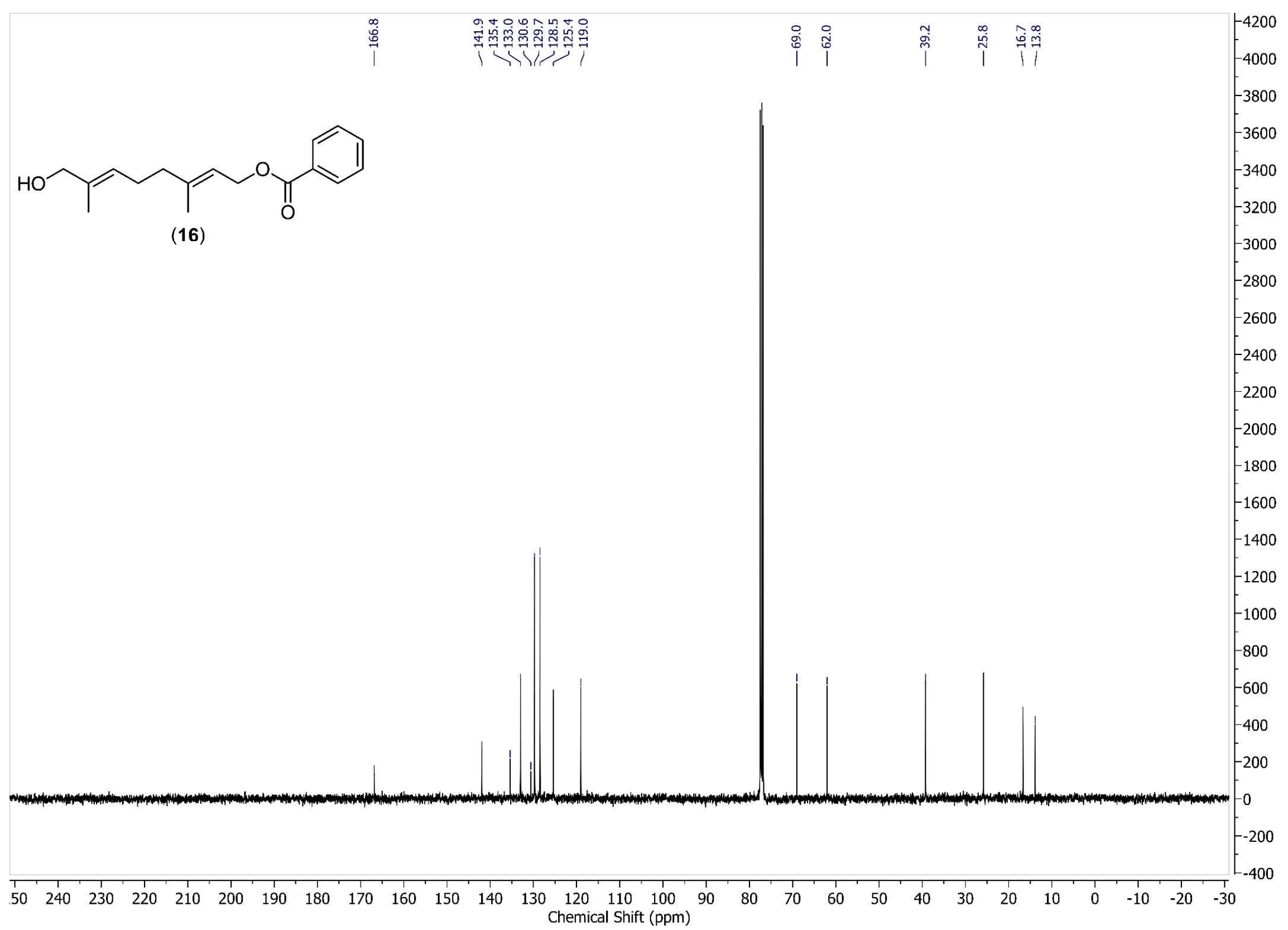

Figure S4: ${ }^{13} \mathrm{C}\left\{{ }^{1} \mathrm{H}\right\}-\mathrm{NMR}\left(101 \mathrm{MHz}, \mathrm{CDCl}_{3}\right.$ ) spectrum of (2E,6E)-8-Hydroxy-3,7-dimethylocta-2,6-dien-1-yl benzoate (16). 


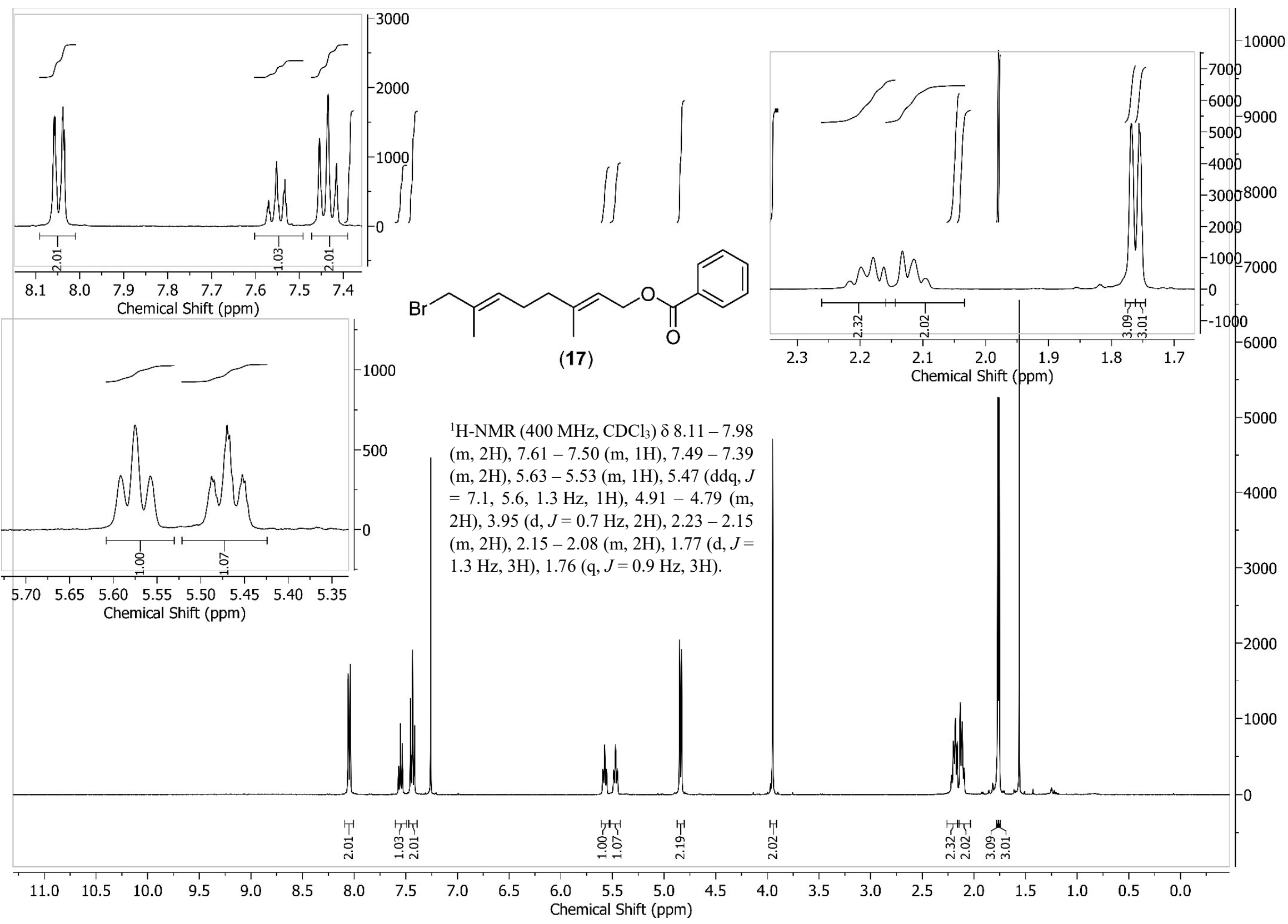

Figure S5: ${ }^{1} \mathrm{H}-\mathrm{NMR}$ spectrum of $(2 E, 6 E)-8-B r o m o-3,7-d i m e t h y l o c t a-2,6-d i e n-1-y l$ benzoate (17). 


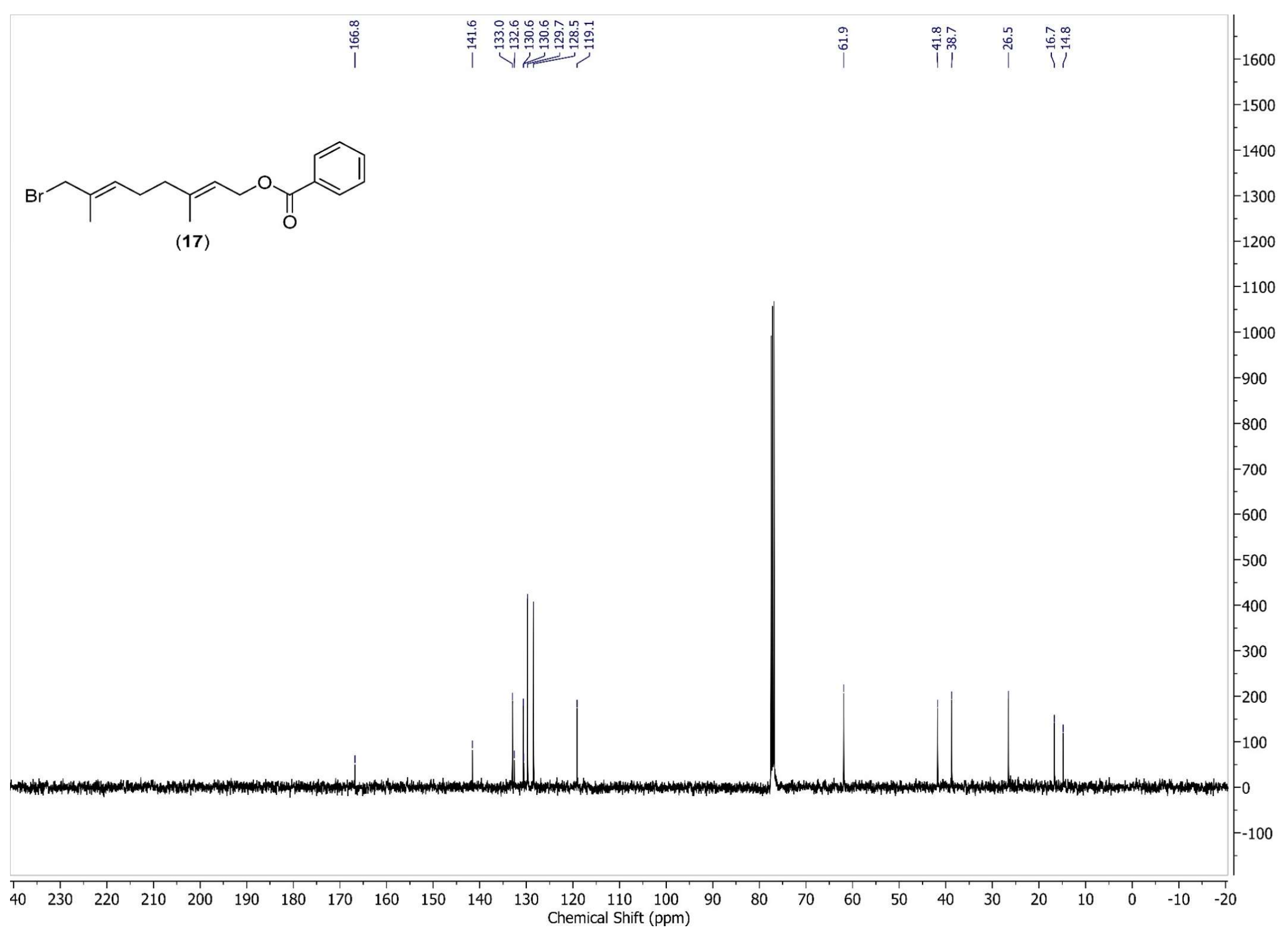

Figure S6: ${ }^{13} \mathrm{C}\left\{{ }^{1} \mathrm{H}\right\}-\mathrm{NMR}\left(101 \mathrm{MHz}, \mathrm{CDCl}_{3}\right)$ spectrum of (2E,6E)-8-Bromo-3,7-dimethylocta-2,6-dien-1-yl benzoate (17). 


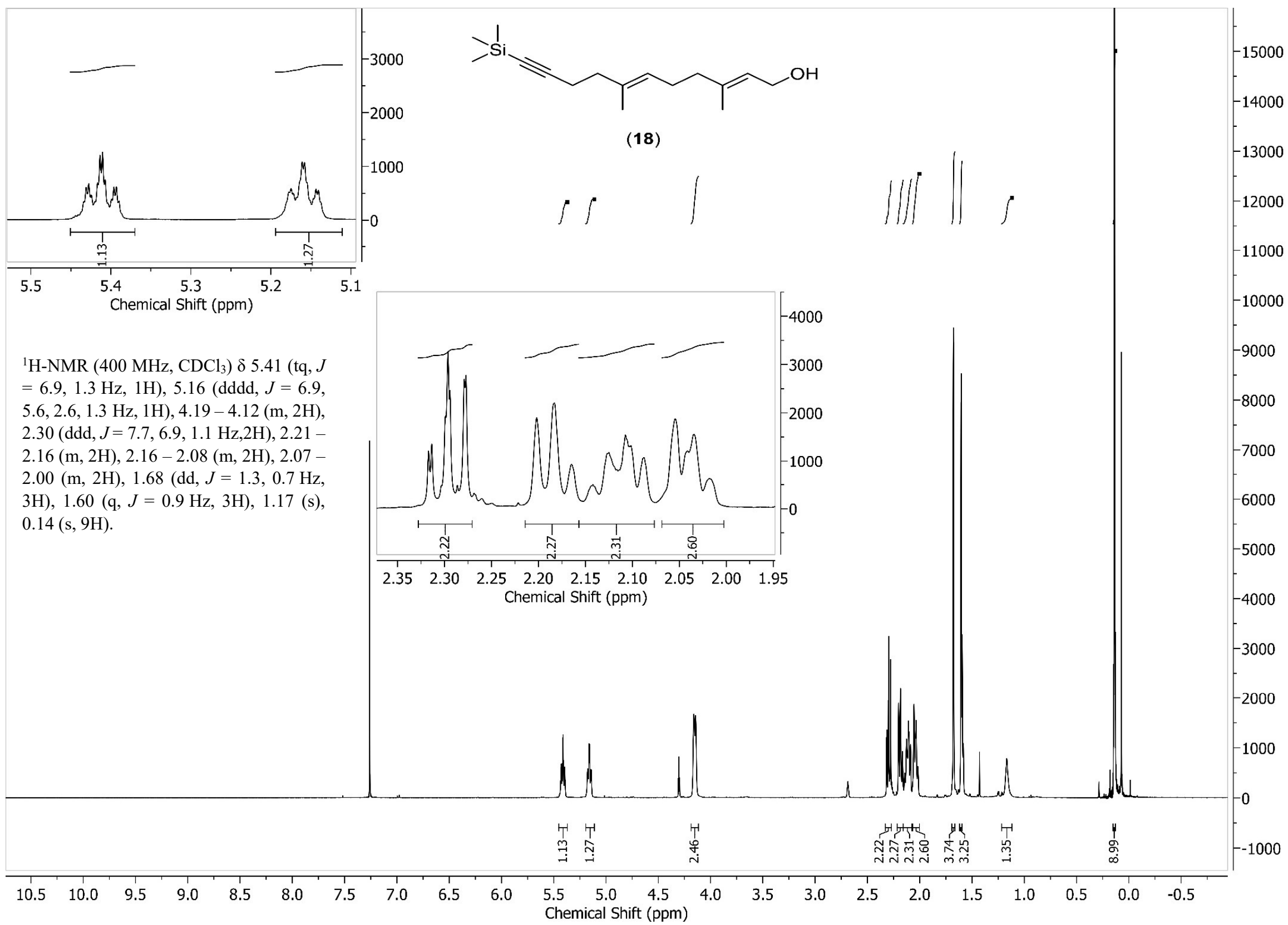

Figure S7: ${ }^{H} \mathrm{H}-\mathrm{NMR}$ spectrum of (2E,6E)-3,7-Dimethyl-11-(trimethylsilyl)undeca-2,6-dien-10-yn-1-ol (18). 


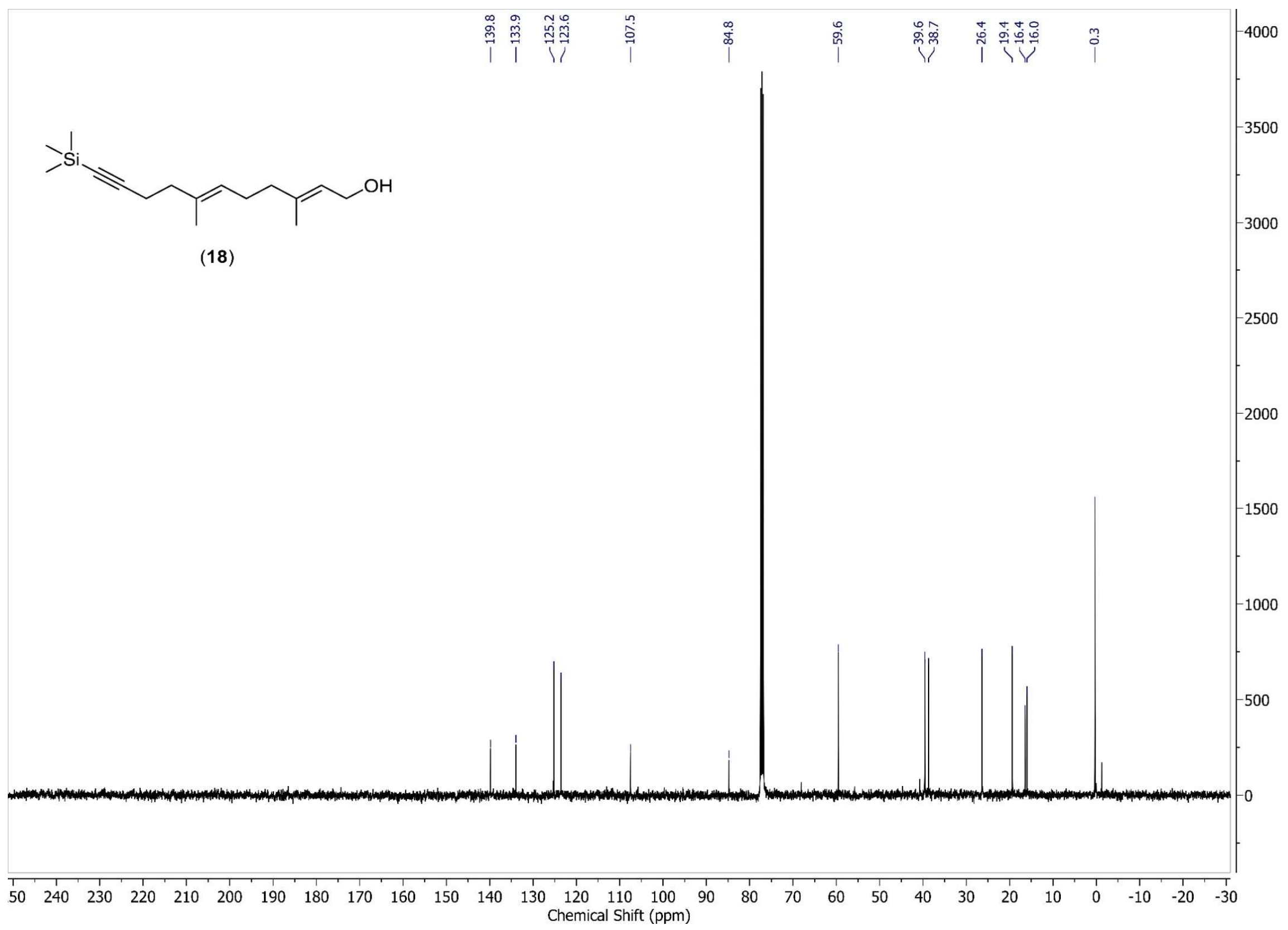

Figure S8: ${ }^{13} \mathrm{C}\left\{{ }^{1} \mathrm{H}\right\}-\mathrm{NMR}\left(101 \mathrm{MHz}, \mathrm{CDCl}_{3}\right)$ spectrum of (2E,6E)-3,7-Dimethyl-11-(trimethylsilyl)undeca-2,6-dien-10-yn-1-ol (18). 


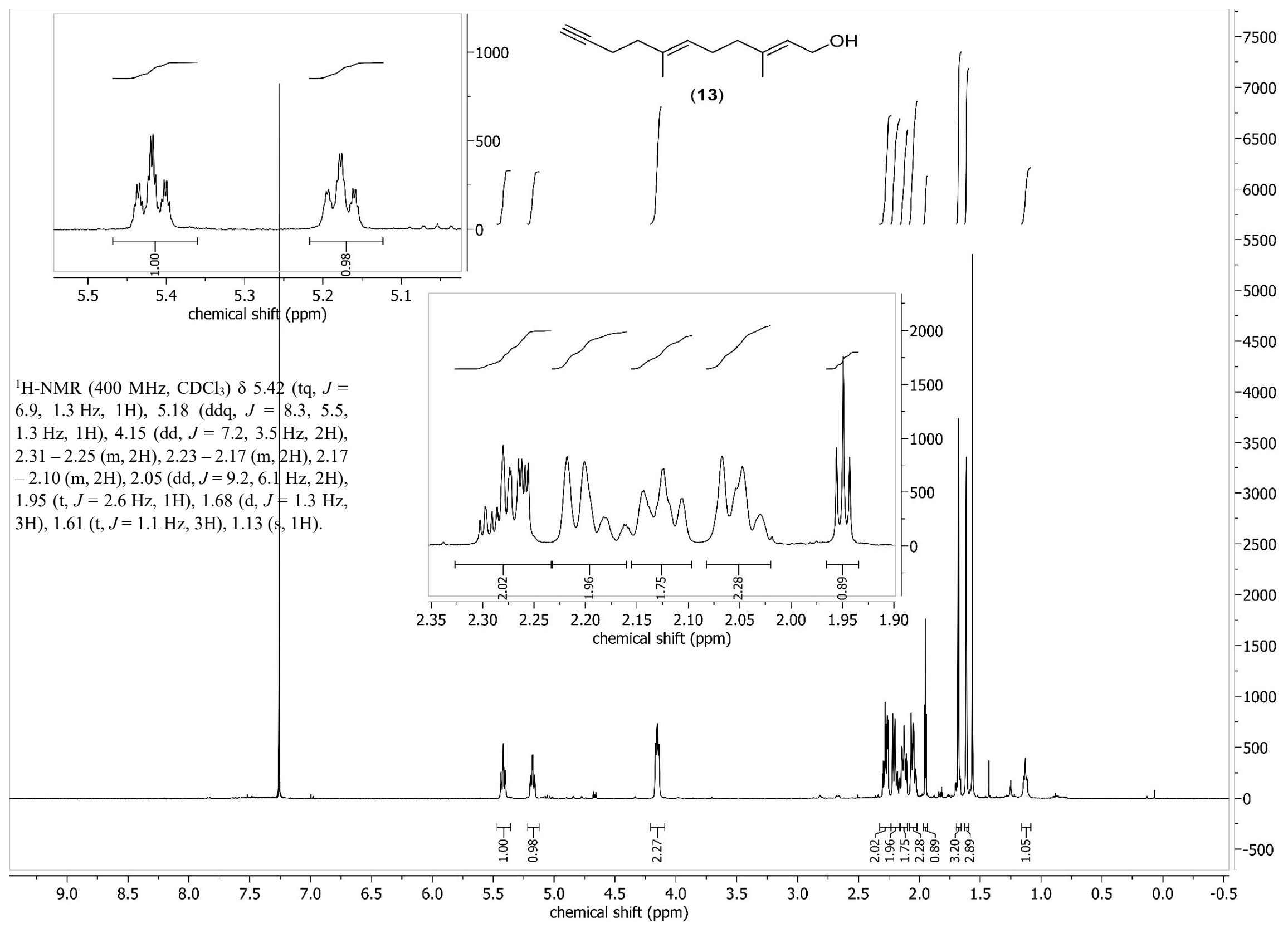

Figure S9: 'H-NMR spectrum of (2E,6E)-3,7-Dimethylundeca-2,6-dien-10-yn-1-ol (13). 


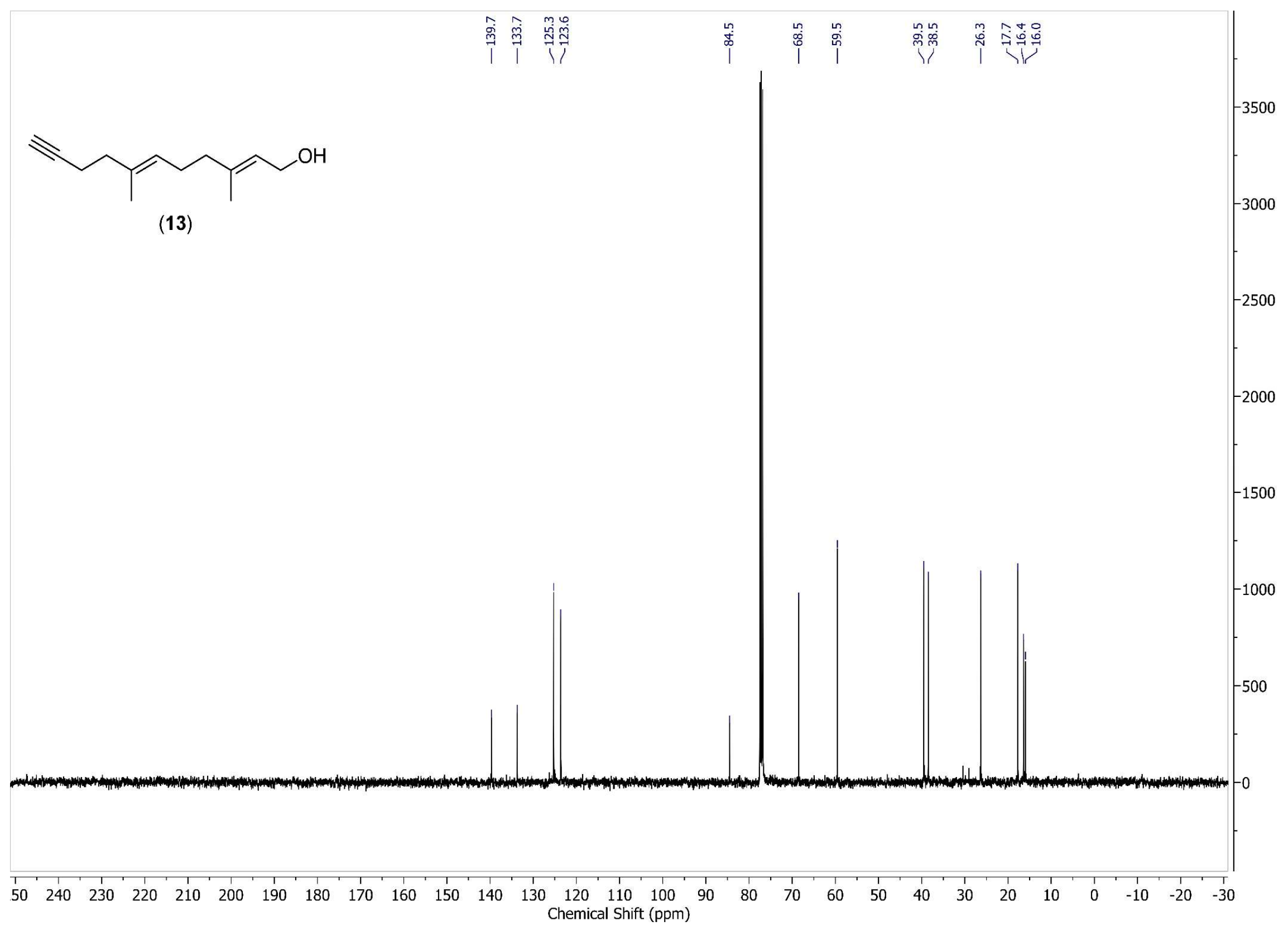

Figure S10: ${ }^{13} \mathrm{C}\left\{{ }^{1} \mathrm{H}\right\}-\mathrm{NMR}\left(101 \mathrm{MHz}, \mathrm{CDCl}_{3}\right)$ spectrum of (2E,6E)-3,7-Dimethylundeca-2,6-dien-10-yn-1-ol (13). 


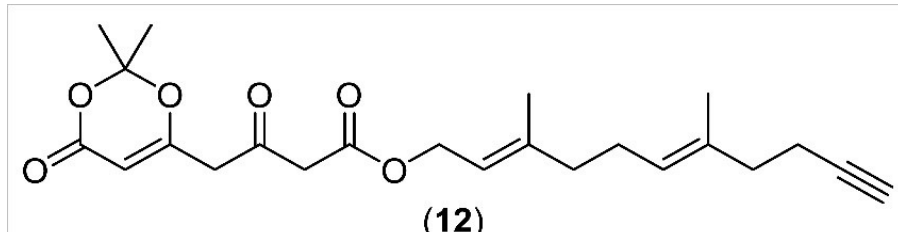

(12)

${ }^{1} \mathrm{H}-\mathrm{NMR}\left(400 \mathrm{MHz}, \mathrm{CDCl}_{3}\right) \delta 5.36(\mathrm{~d}, J=0.6 \mathrm{~Hz}, 1 \mathrm{H}), 5.33$ (tq, $J=7.2,1.3 \mathrm{~Hz}, 1 \mathrm{H}), 5.15(\mathrm{ddd}, J=5.4,4.1,2.8 \mathrm{~Hz}, 1 \mathrm{H}), 4.66(\mathrm{~d}$, $J=7.3 \mathrm{~Hz}, 2 \mathrm{H}), 3.51(\mathrm{~s}, 2 \mathrm{H}), 3.50(\mathrm{~s}, 2 \mathrm{H}), 2.31-2.24(\mathrm{~m}, 2 \mathrm{H})$, $2.23-2.16(\mathrm{~m}, 2 \mathrm{H}), 2.15-2.10(\mathrm{~m}, 2 \mathrm{H}), 2.10-2.04(\mathrm{~m}, 2 \mathrm{H})$, $1.94(\mathrm{t}, J=2.5 \mathrm{~Hz}, 1 \mathrm{H}), 1.71(\mathrm{~s}, 9 \mathrm{H}), 1.61(\mathrm{~d}, J=1.3 \mathrm{~Hz}, 3 \mathrm{H})$.
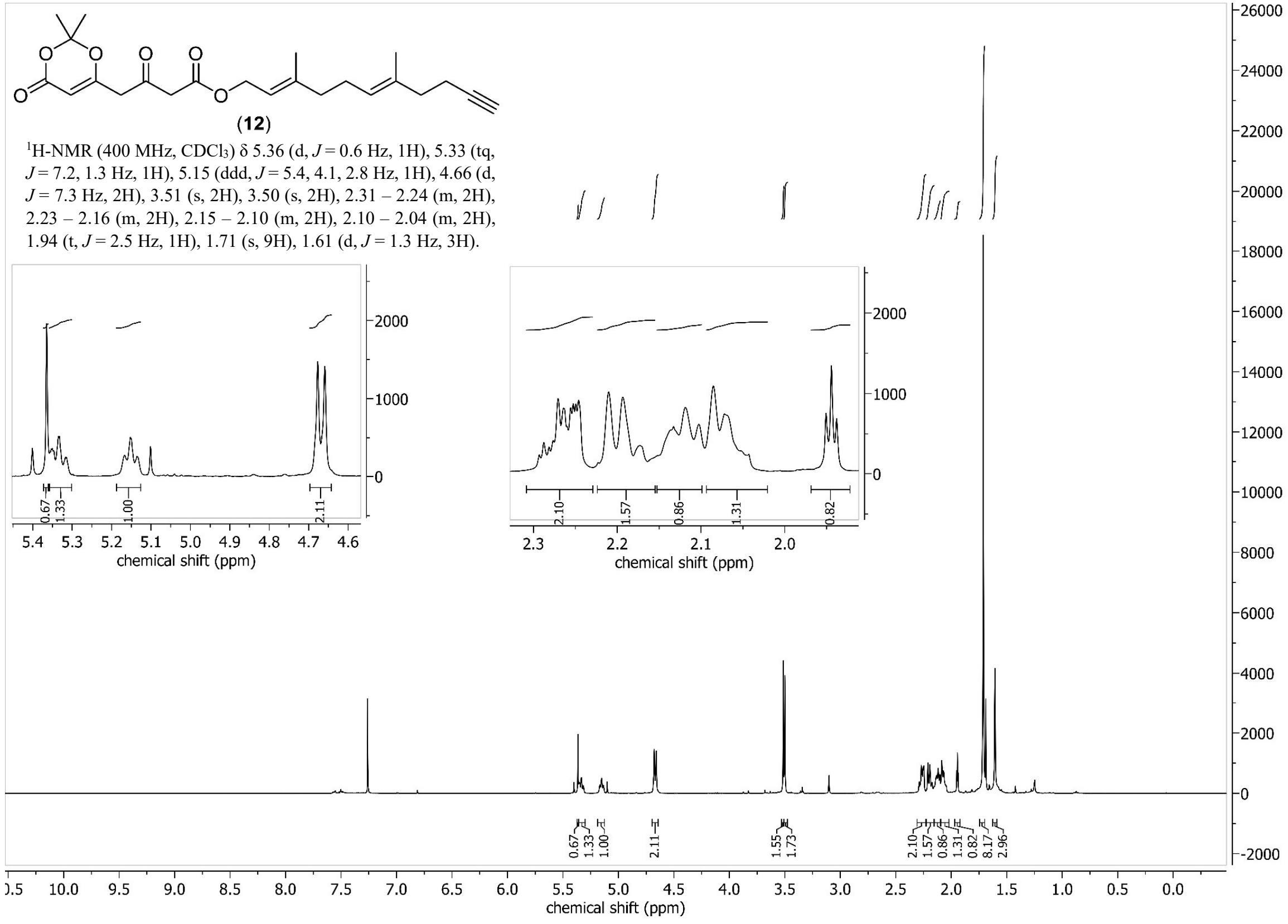

Figure S11: 'H-NMR spectrum of (2E,6E)-3,7-Dimethylundeca-2,6-dien-10-yn-1-yl 4-(2,2-dimethyl-4-oxo-4H-1,3-dioxin-6-yl)-3-oxobutanoate (12). 


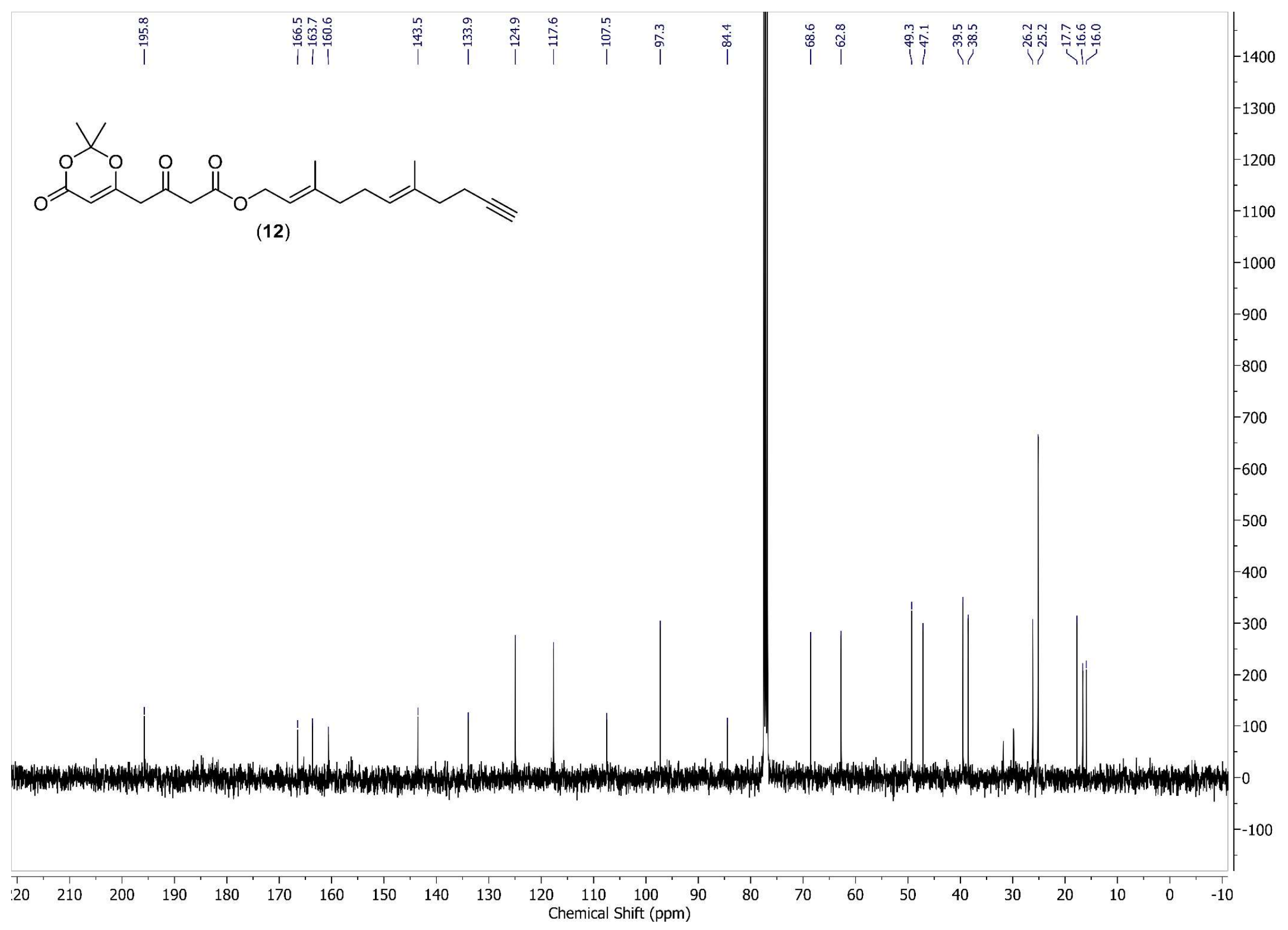

Figure S12: ${ }^{13} \mathrm{C}\{\mathrm{H}\}$-NMR $\left(101 \mathrm{MHz}, \mathrm{CDCl}_{3}\right)$ spectrum of $(2 E, 6 E)-3,7-$-Dimethylundeca-2,6-dien-10-yn-1-yl 4-(2,2-dimethyl-4-oxo-4H-1,3-dioxin-6-yl)-3-oxobutanoate (12). 


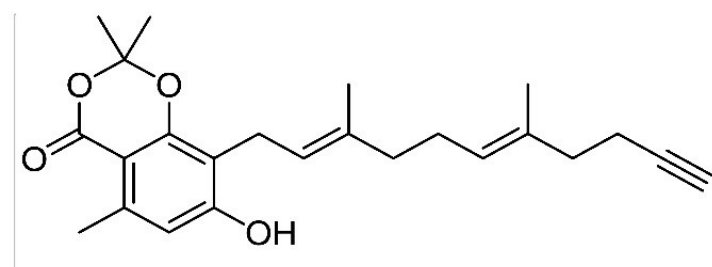

(9)

${ }^{1} \mathrm{H}-\mathrm{NMR}\left(400 \mathrm{MHz}, \mathrm{CDCl}_{3}\right) \delta 6.40(\mathrm{~d}, J=0.9 \mathrm{~Hz}, 1 \mathrm{H}), 5.91(\mathrm{~s}$, $1 \mathrm{H}, \mathrm{OH}), 5.22-5.15(\mathrm{~m}, 1 \mathrm{H}), 5.12(\mathrm{tt}, J=5.6,1.4 \mathrm{~Hz}, 1 \mathrm{H}), 3.32$ $(\mathrm{d}, J=7.3 \mathrm{~Hz}, 2 \mathrm{H}), 2.59(\mathrm{~d}, J=0.8 \mathrm{~Hz}, 3 \mathrm{H}), 2.29-2.21(\mathrm{~m}, 2 \mathrm{H})$, $2.21-2.15(\mathrm{~m}, 2 \mathrm{H}), 2.15-2.09(\mathrm{~m}, 2 \mathrm{H}), 2.06(\mathrm{dt}, J=7.3,3.1 \mathrm{~Hz}$ 2H), 1.93 (t, $J=2.5 \mathrm{~Hz}, 1 \mathrm{H}), 1.79$ (d, $J=1.3 \mathrm{~Hz}, 3 \mathrm{H}), 1.69$ (s, $6 \mathrm{H}), 1.59(\mathrm{~d}, J=1.3 \mathrm{~Hz}, 3 \mathrm{H})$.
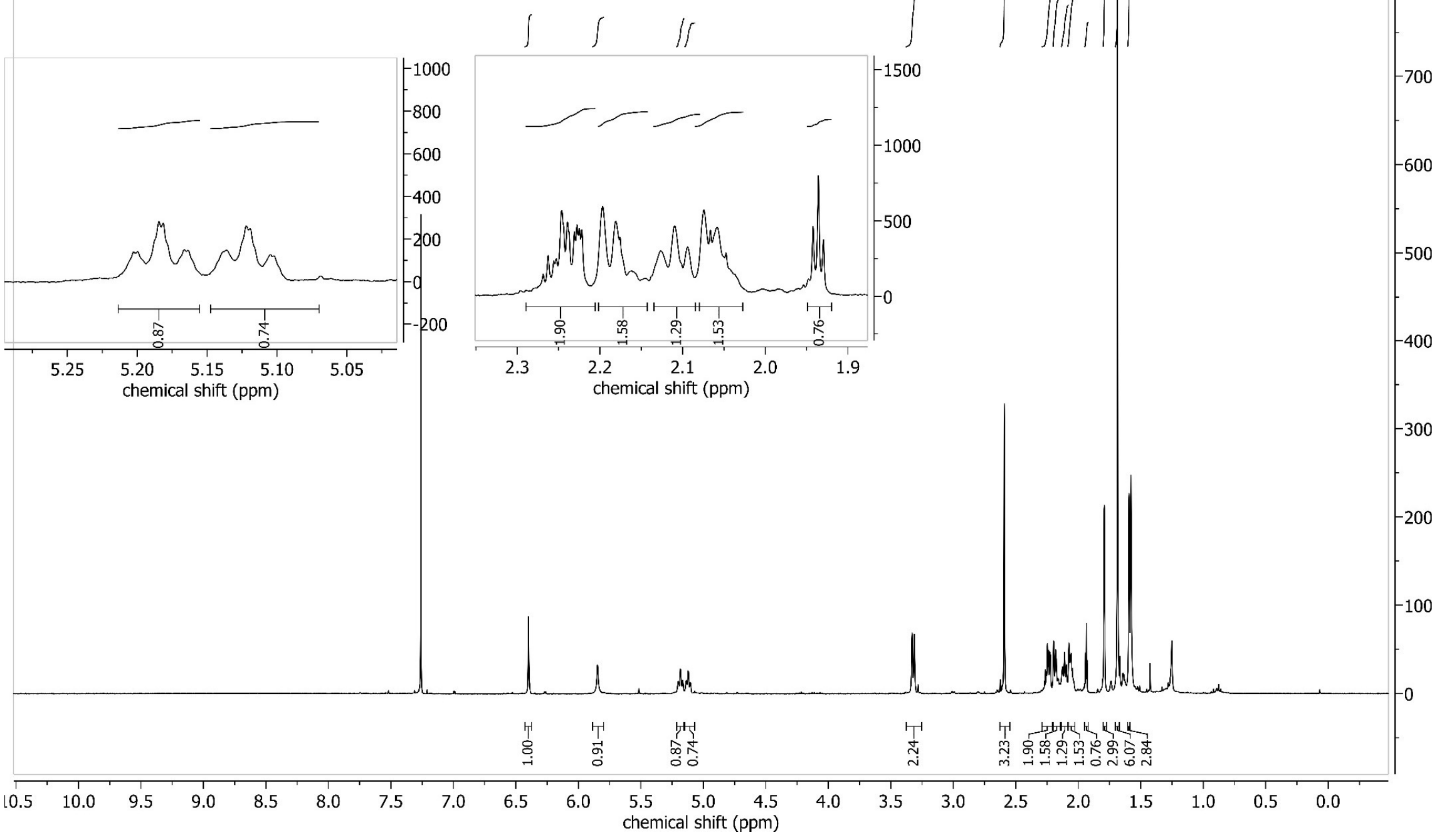

Figure S13: 'H-NMR spectrum of 8-((2E,6E)-3,7-Dimethylundeca-2,6-dien-10-yn-1-yl)-7-hydroxy-2,2,5-trimethyl-4H-benzo[d][1,3]dioxin-4-one (9). 


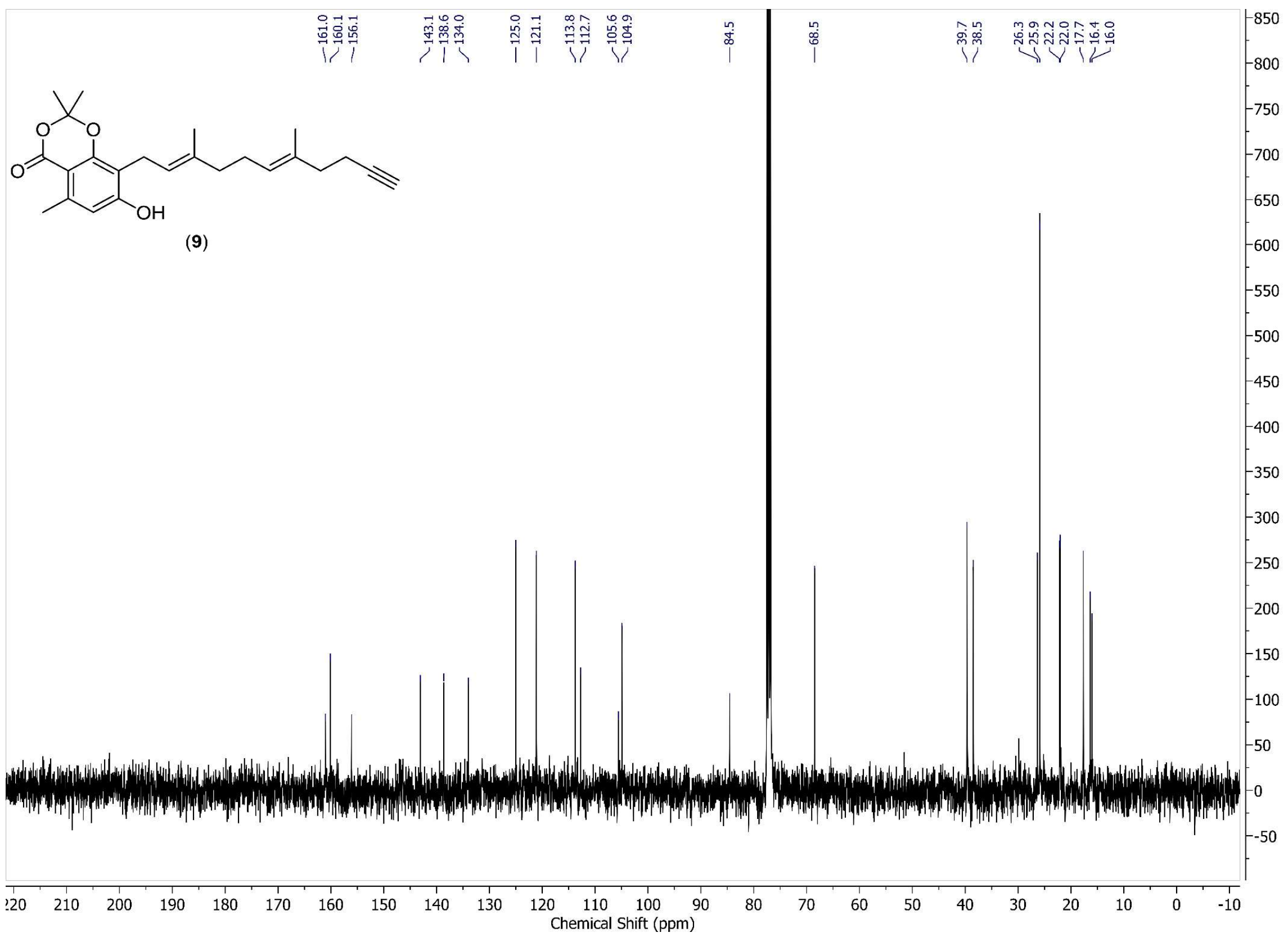

Figure S14: ${ }^{13} \mathrm{C}\left\{{ }^{1} \mathrm{H}\right\}-\mathrm{NMR}\left(101 \mathrm{MHz}, \mathrm{CDCl}_{3}\right)$ spectrum of 8-((2E,6E)-3,7-Dimethylundeca-2,6-dien-10-yn-1-yl)-7-hydroxy-2,2,5-trimethyl-4H-benzo[d][1,3]dioxin-4-one (9). 


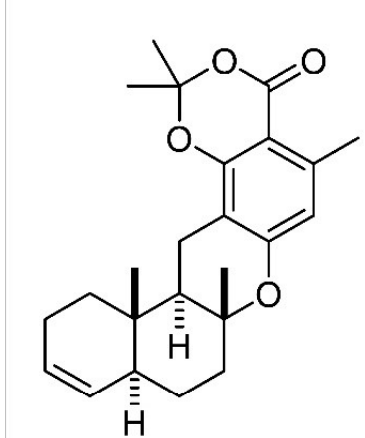

(8)

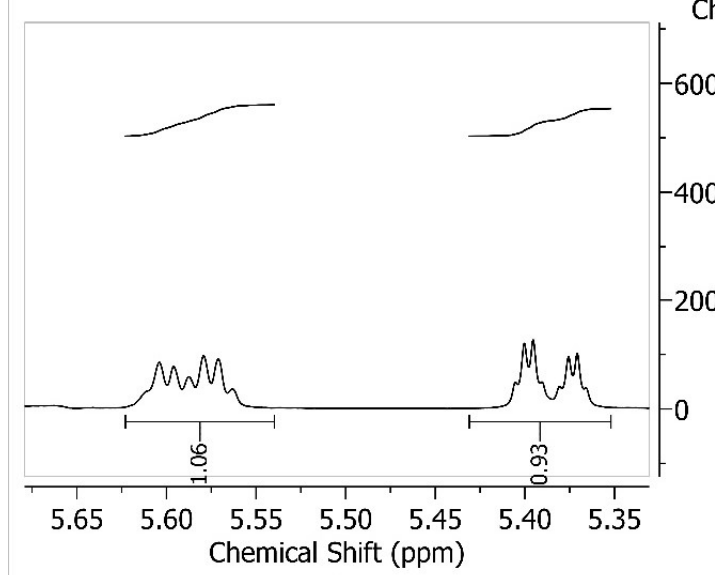

Chemical Shift (ppm)

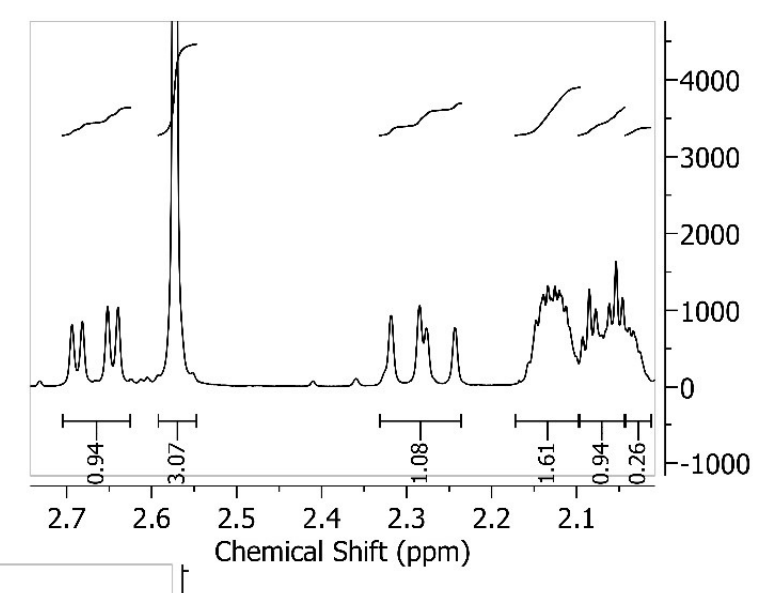

6000

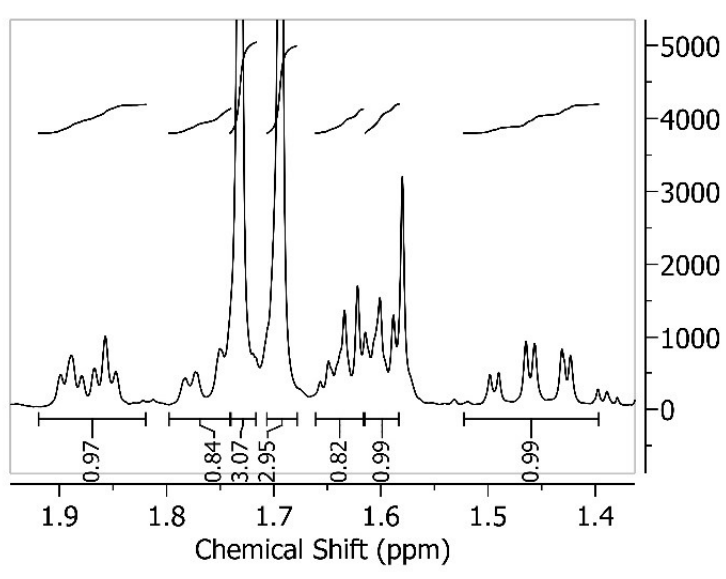

${ }^{1} \mathrm{H}-\mathrm{NMR}\left(400 \mathrm{MHz}, \mathrm{CDCl}_{3}\right) \delta 6.34(\mathrm{~d}, J=0.9 \mathrm{~Hz}, 1 \mathrm{H}), 5.58$ $4000(\mathrm{dq}, J=10.0,3.2 \mathrm{~Hz}, 1 \mathrm{H}), 5.38(\mathrm{dq}, J=9.9,2.1 \mathrm{~Hz}, 1 \mathrm{H})$, $2.66(\mathrm{dd}, J=16.8,4.9 \mathrm{~Hz}, 1 \mathrm{H}), 2.57(\mathrm{~s}, 3 \mathrm{H}), 2.33-2.23(\mathrm{~m}$, $20001 \mathrm{H}), 2.13(\mathrm{ddq}, J=5.2,3.9,2.7,1.9 \mathrm{~Hz}, 2 \mathrm{H}), 2.07(\mathrm{dt}, J=$ $12.9,3.4 \mathrm{~Hz}, 1 \mathrm{H}), 2.03(\mathrm{dt}, J=3.2,1.6 \mathrm{~Hz}, 1 \mathrm{H}), 1.87(\mathrm{dt}, J$ $=12.8,4.0 \mathrm{~Hz}, 1 \mathrm{H}), 1.79-1.70(\mathrm{~m}, 1 \mathrm{H}), 1.73(\mathrm{~s}, 3 \mathrm{H}), 1.69$ $(\mathrm{s}, 3 \mathrm{H}), 1.66-1.62(\mathrm{~m}, 1 \mathrm{H}), 1.59(\mathrm{td}, J=6.5,5.9,3.7 \mathrm{~Hz}$ $1 \mathrm{H}), 1.50-1.42(\mathrm{~m}, 1 \mathrm{H}), 1.27(\mathrm{~d}, J=1.0 \mathrm{~Hz}, 3 \mathrm{H}), 1.22$ (dd, $J=5.0,4.1 \mathrm{~Hz}, 1 \mathrm{H}), 0.82(\mathrm{~d}, J=0.8 \mathrm{~Hz}, 3 \mathrm{H})$.

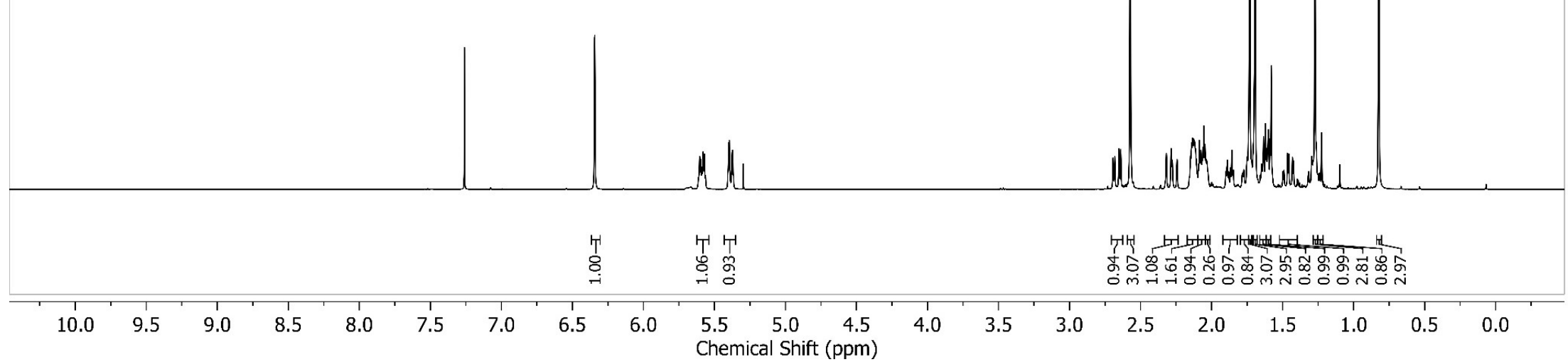

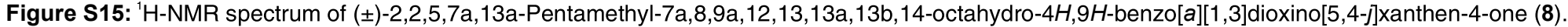



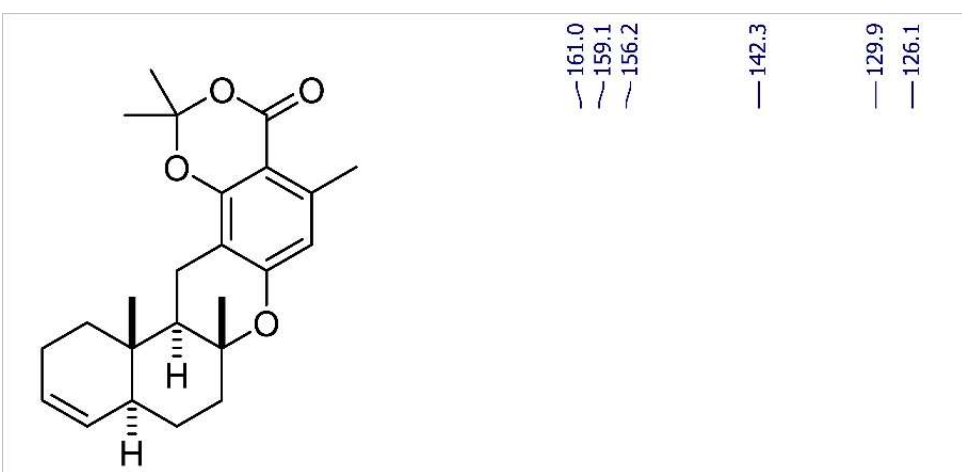

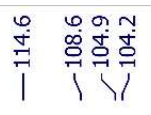
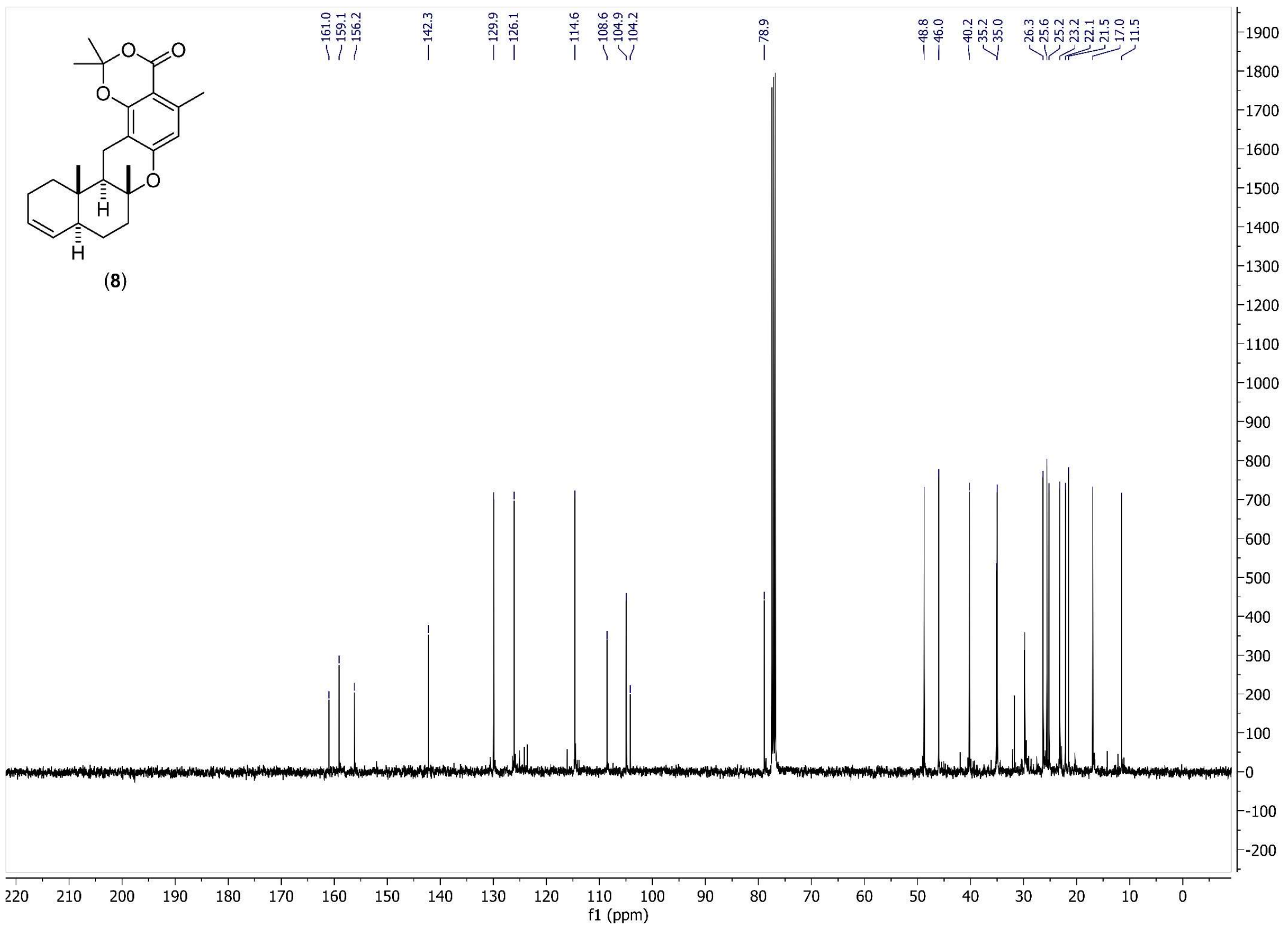

Figure S16: ${ }^{13} \mathrm{C}\left\{{ }^{1} \mathrm{H}\right\}$-NMR $\left(101 \mathrm{MHz}, \mathrm{CDCl}_{3}\right)$ spectrum of $( \pm)-2,2,5,7 \mathrm{a}, 13 \mathrm{a}-$ Pentamethyl-7a,8,9a,12,13,13a,13b,14-octahydro-4H,9H-benzo[a][1,3]dioxino[5,4-j]xanthen-4-one (8). 


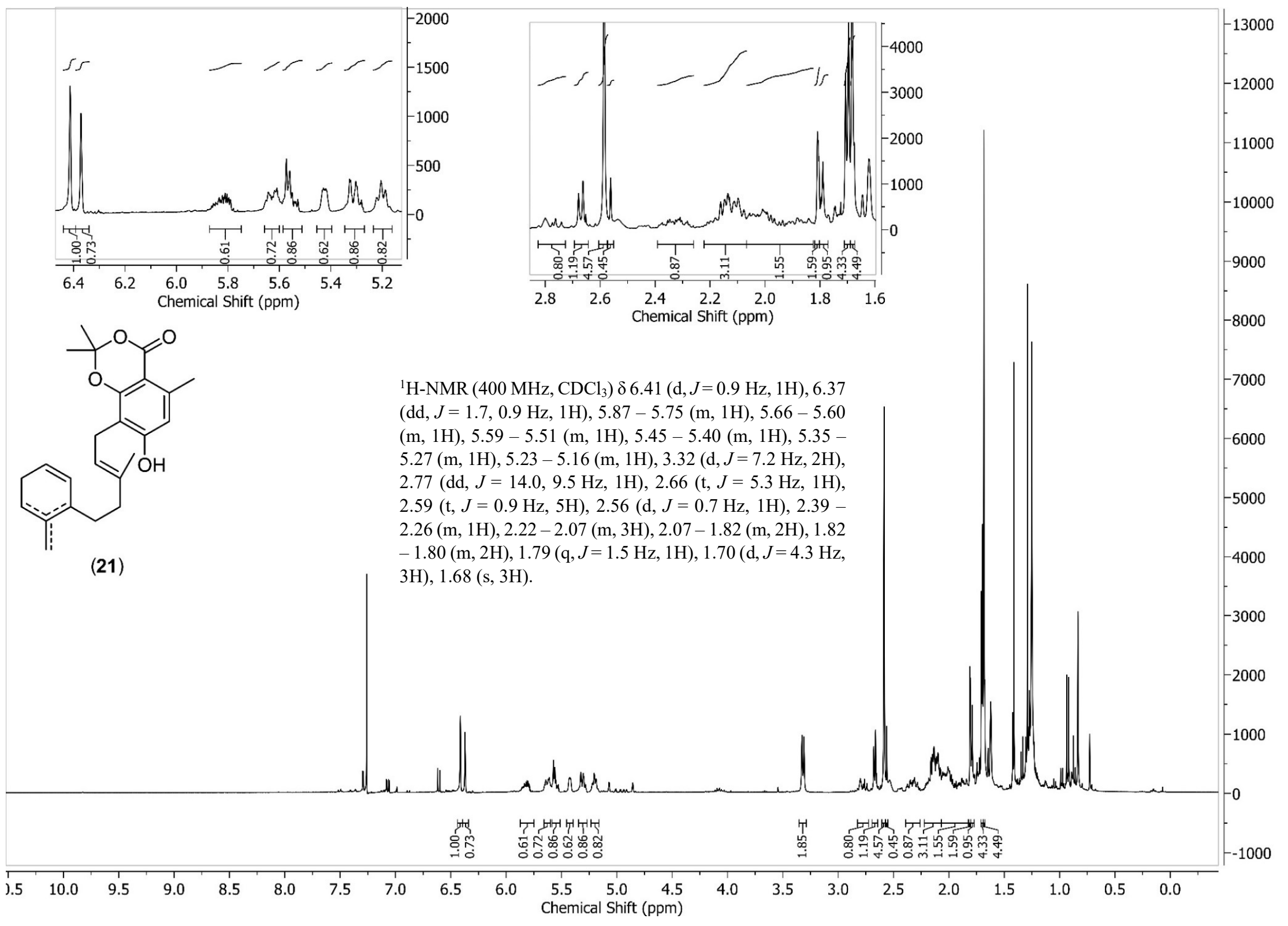

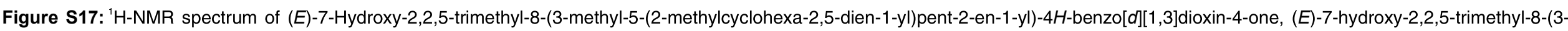

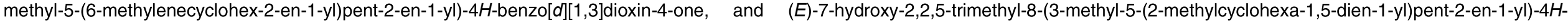
benzo[d][1,3]dioxin-4-one (21). 


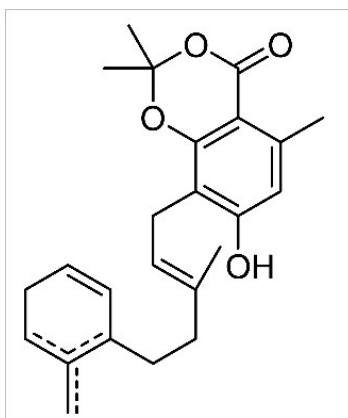

(21)

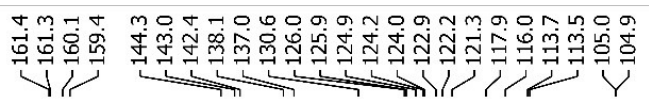

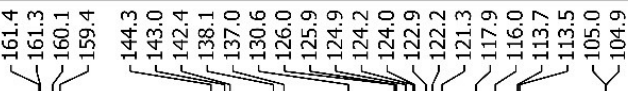

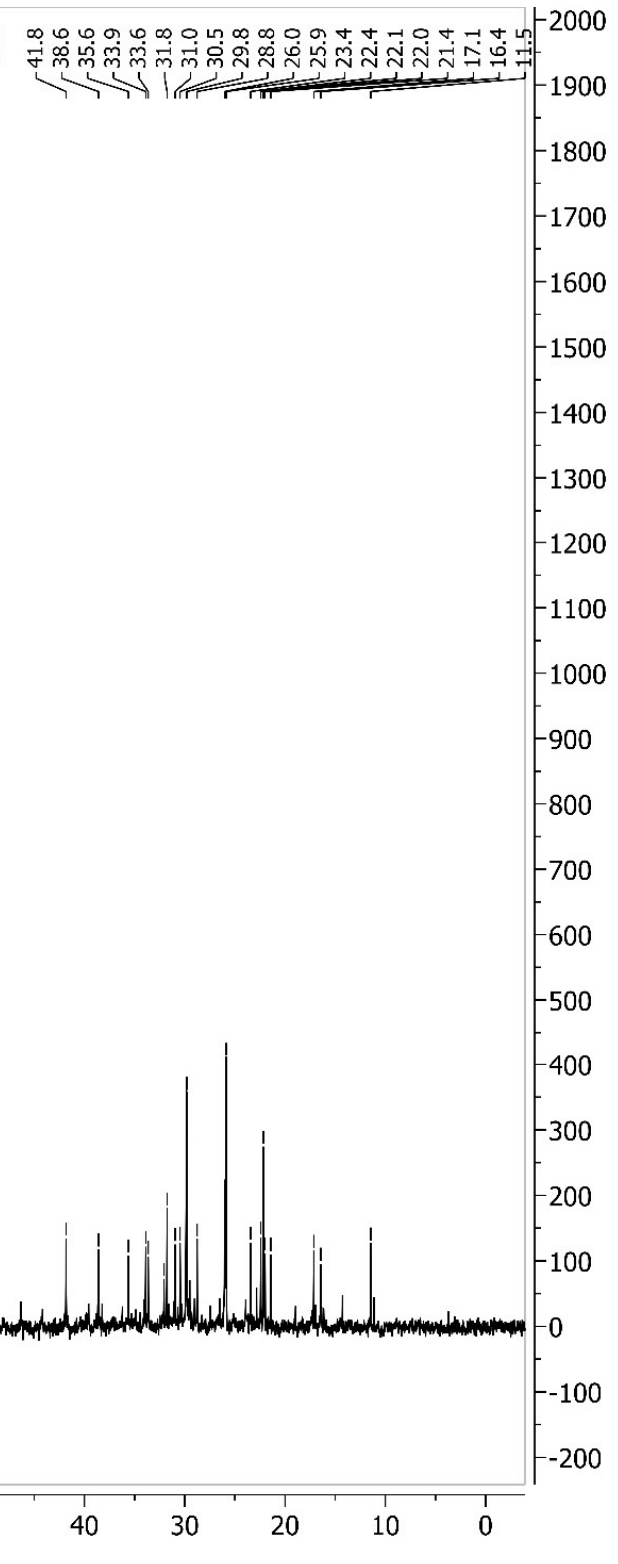

200

$190 \quad 180$

$160 \quad 150$
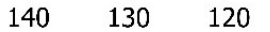

$\begin{array}{lrr}110 & 100 & 90 \\ \text { Chemical Shift } & (\mathrm{ppm})\end{array}$

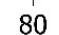

70

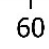

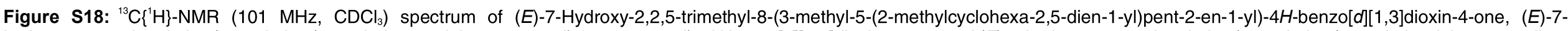

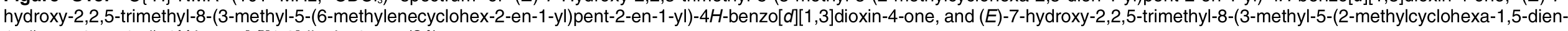
1-yl)pent-2-en-1-yl)-4H-benzo[d][1,3]dioxin-4-one (21). 


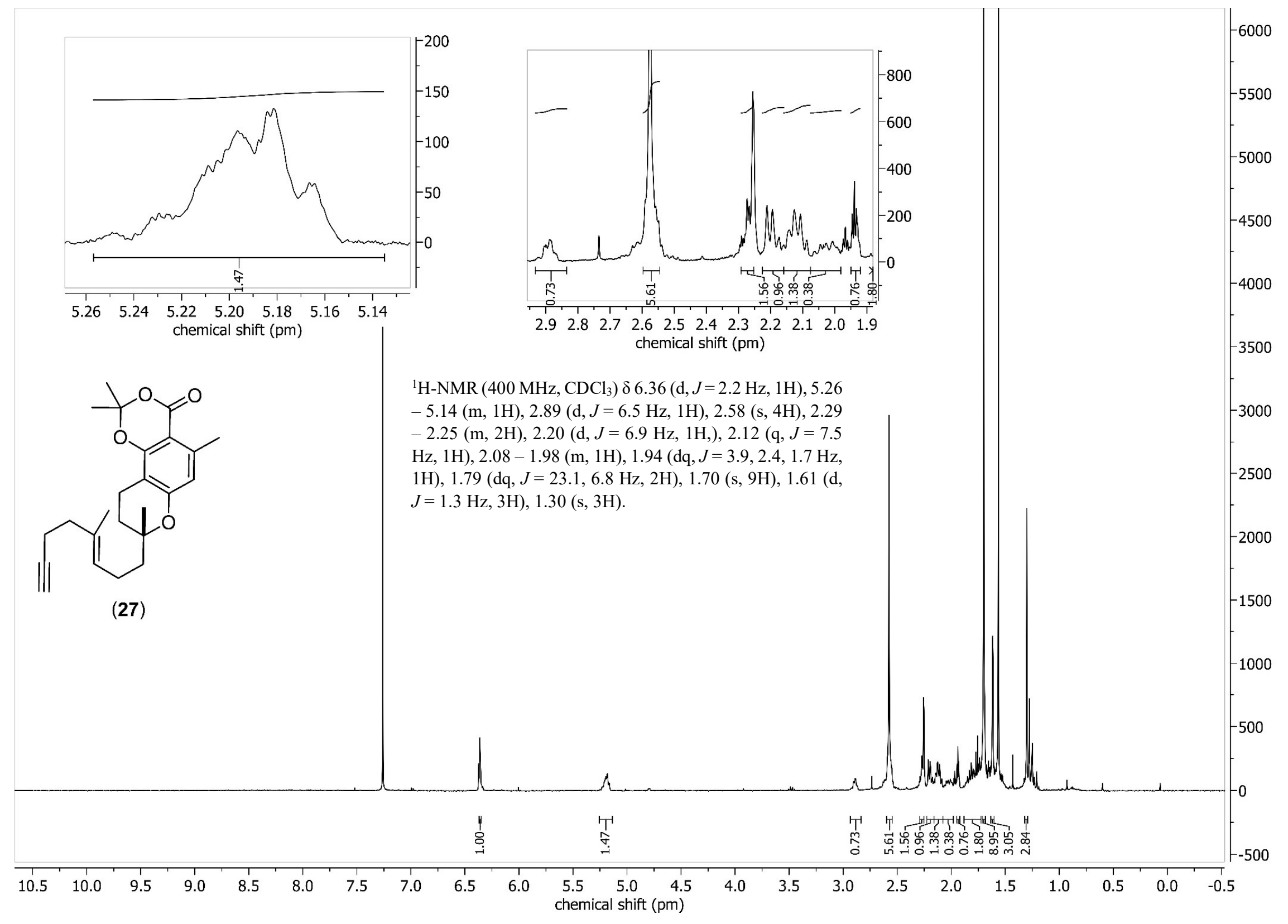

Figure S19: ${ }^{1} \mathrm{H}-\mathrm{NMR}$ spectrum of $( \pm)-2,2,5,8$-tetramethyl-8-(4-methyloct-3-en-7-yn-1-yl)-9,10-dihydro-4H,8H-[1,3]dioxino[4,5-f]chromen-4-one (27). 

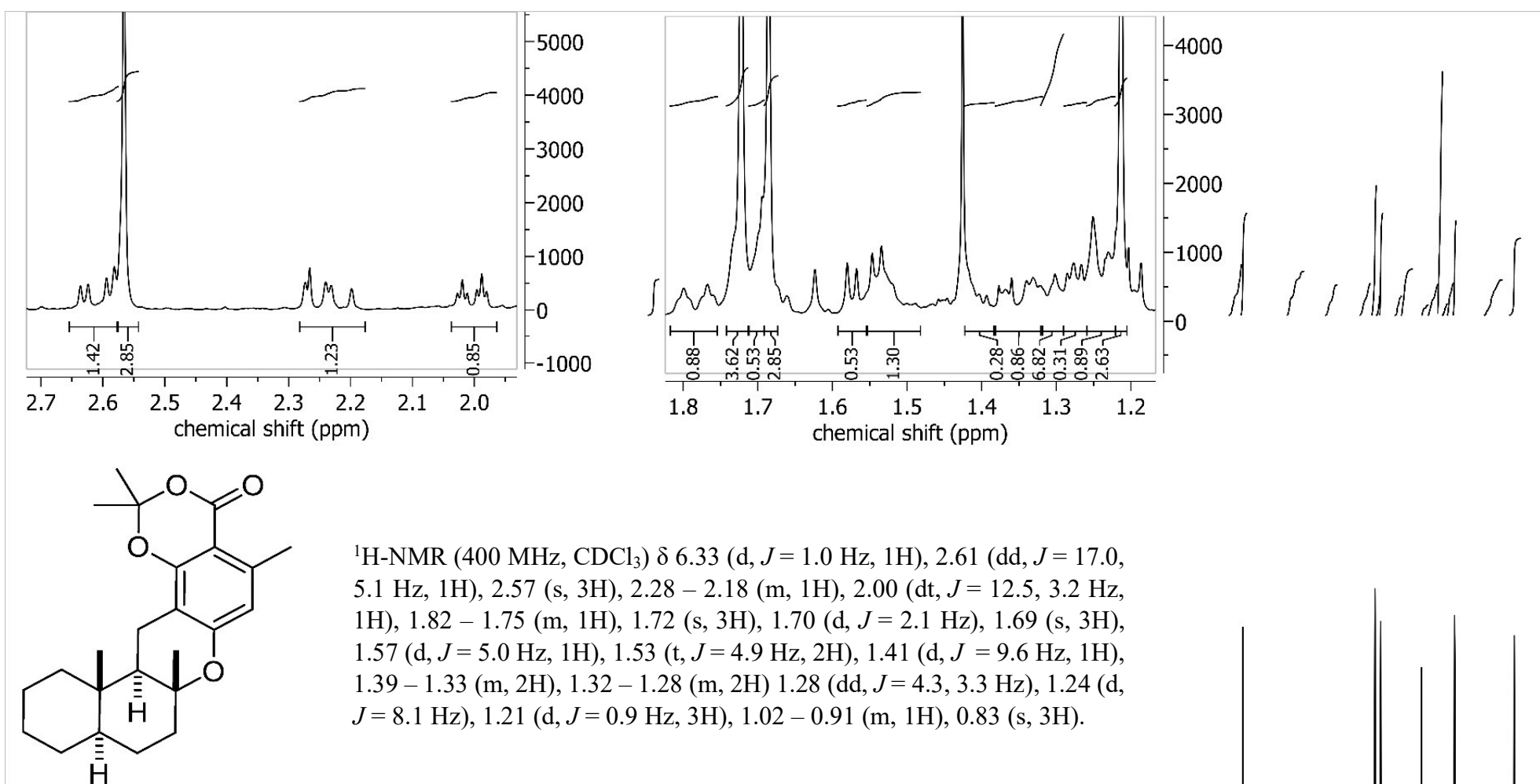

${ }^{1} \mathrm{H}-\mathrm{NMR}\left(400 \mathrm{MHz}, \mathrm{CDCl}_{3}\right) \delta 6.33(\mathrm{~d}, J=1.0 \mathrm{~Hz}, 1 \mathrm{H}), 2.61(\mathrm{dd}, J=17.0$, $5.1 \mathrm{~Hz}, 1 \mathrm{H}), 2.57$ (s, 3H), $2.28-2.18(\mathrm{~m}, 1 \mathrm{H}), 2.00(\mathrm{dt}, J=12.5,3.2 \mathrm{~Hz}$, $1 \mathrm{H}), 1.82-1.75(\mathrm{~m}, 1 \mathrm{H}), 1.72(\mathrm{~s}, 3 \mathrm{H}), 1.70(\mathrm{~d}, J=2.1 \mathrm{~Hz}), 1.69(\mathrm{~s}, 3 \mathrm{H})$, $1.57(\mathrm{~d}, J=5.0 \mathrm{~Hz}, 1 \mathrm{H}), 1.53(\mathrm{t}, J=4.9 \mathrm{~Hz}, 2 \mathrm{H}), 1.41(\mathrm{~d}, J=9.6 \mathrm{~Hz}, 1 \mathrm{H})$ $1.39-1.33(\mathrm{~m}, 2 \mathrm{H}), 1.32-1.28(\mathrm{~m}, 2 \mathrm{H}) 1.28(\mathrm{dd}, J=4.3,3.3 \mathrm{~Hz}), 1.24(\mathrm{~d}$, $J=8.1 \mathrm{~Hz}), 1.21(\mathrm{~d}, J=0.9 \mathrm{~Hz}, 3 \mathrm{H}), 1.02-0.91(\mathrm{~m}, 1 \mathrm{H}), 0.83(\mathrm{~s}, 3 \mathrm{H})$

(29)

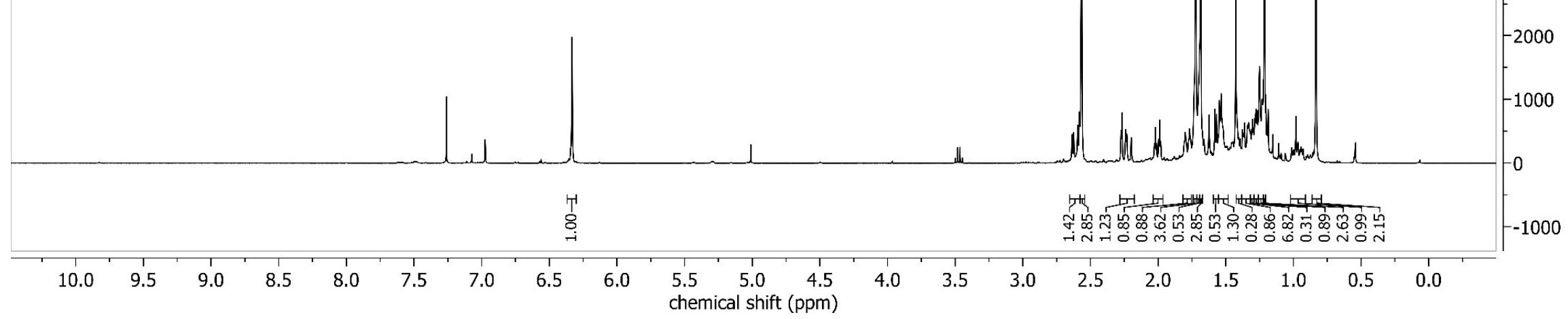

Figure S20: 'H-NMR spectrum of $( \pm)-2,2,5,7 a, 13 a-P e n t a m e t h y l-7 a, 8,9 a, 10,11,12,13,13 a, 13 b, 14-d e c a h y d r o-4 H, 9 H$-benzo[a] $[1,3] d$ dioxino[5,4-]xanthen-4-one (29). 


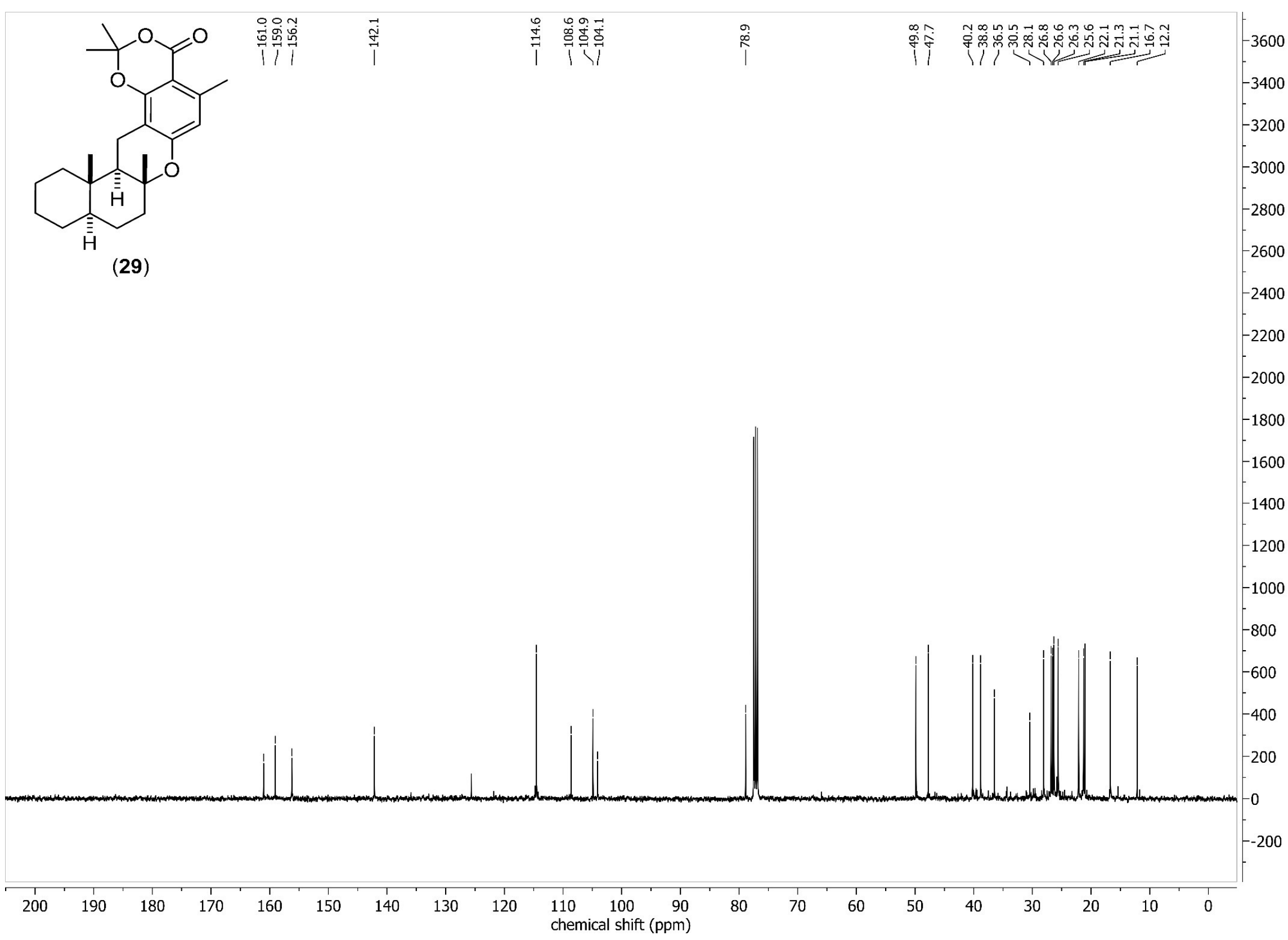

Figure S21: ${ }^{13} \mathrm{C}\left\{{ }^{1} \mathrm{H}\right\}-\mathrm{NMR}\left(101 \mathrm{MHz}, \mathrm{CDCl}_{3}\right)$ spectrum of $( \pm)-2,2,5,7 \mathrm{a}, 13 \mathrm{a}-$-Pentamethyl-7a,8,9a,10,11,12,13,13a, 13b, 14-decahydro-4H,9H-benzo[a][1,3]dioxino[5,4-j]xanthen-4-one (29). 


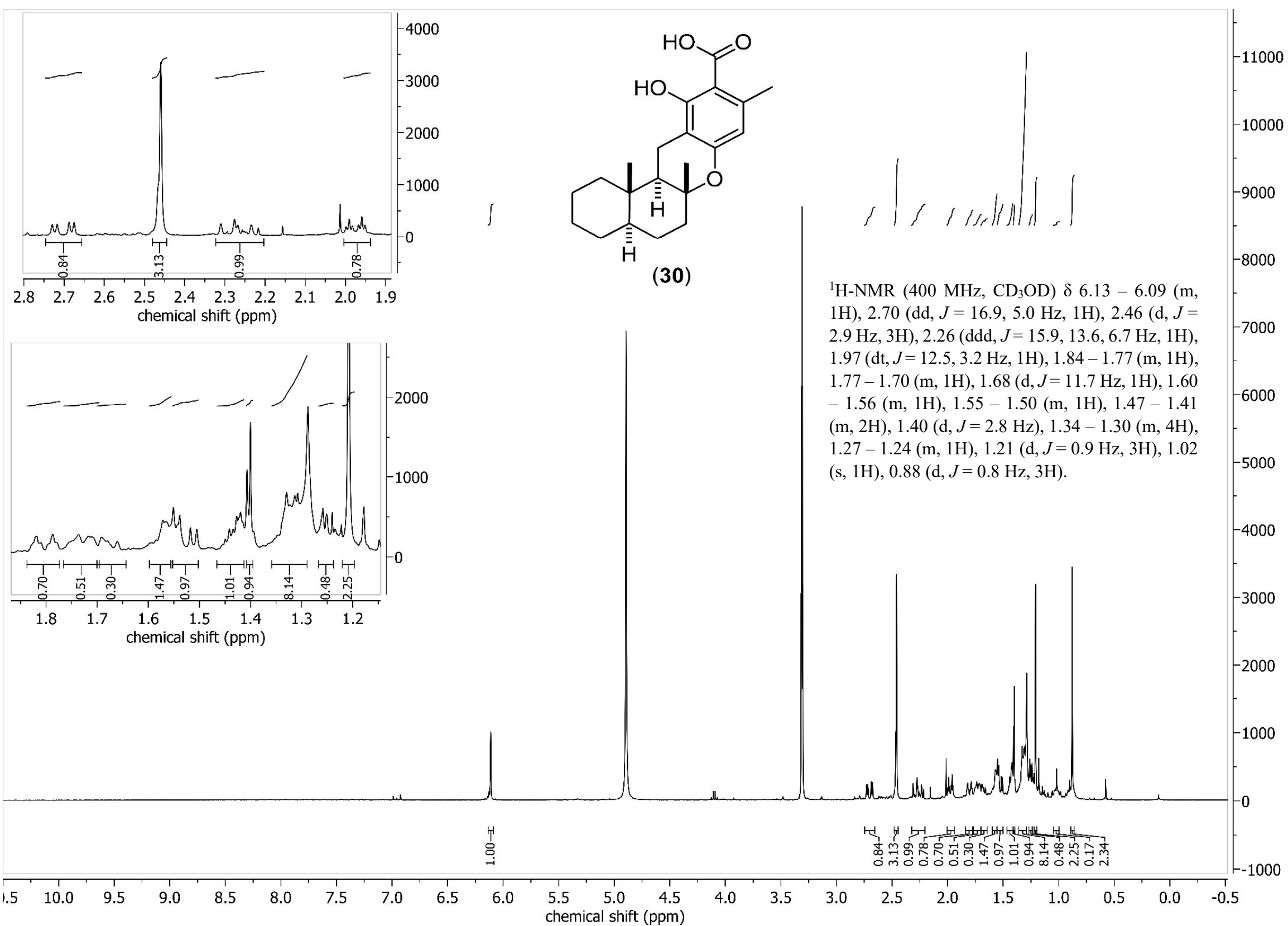

Figure S22: 'H-NMR spectrum of ( \pm )-11-Hydroxy-6a,9,12b-trimethyl-1,3,4,4a,5,6,6a,12,12a,12b-decahydro-2H-benzo[a]xanthene-10-carboxylic acid (30). 


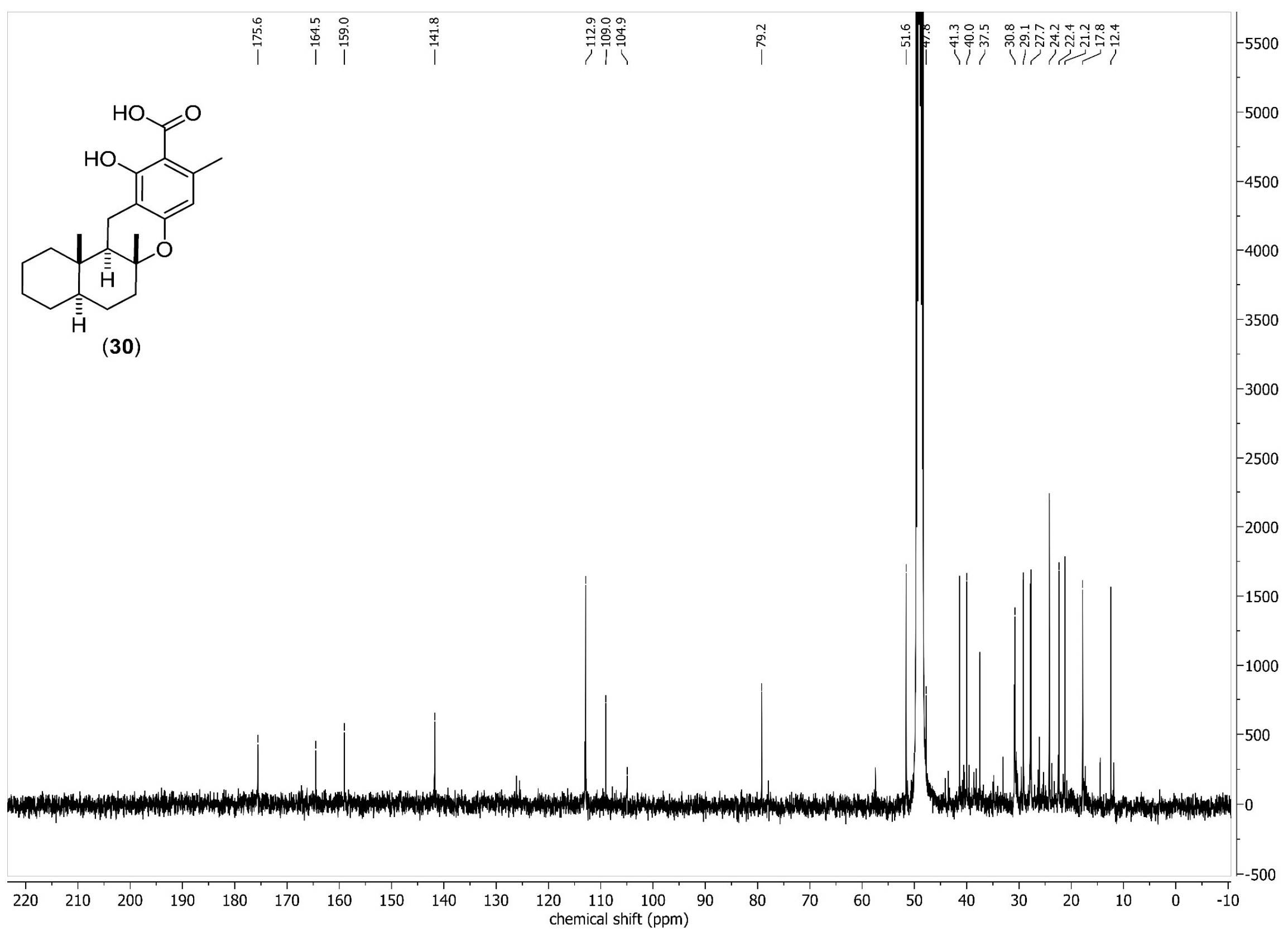

Figure S23: ${ }^{13} \mathrm{C}\{\mathrm{H}\}$-NMR (101 MHz, $\left.\mathrm{CD}_{3} \mathrm{OD}\right)$ spectrum of ( \pm )-11-Hydroxy-6a,9,12b-trimethyl-1,3,4,4a,5,6,6a,12,12a,12b-decahydro-2H-benzo[a]xanthene-10-carboxylic acid (30). 


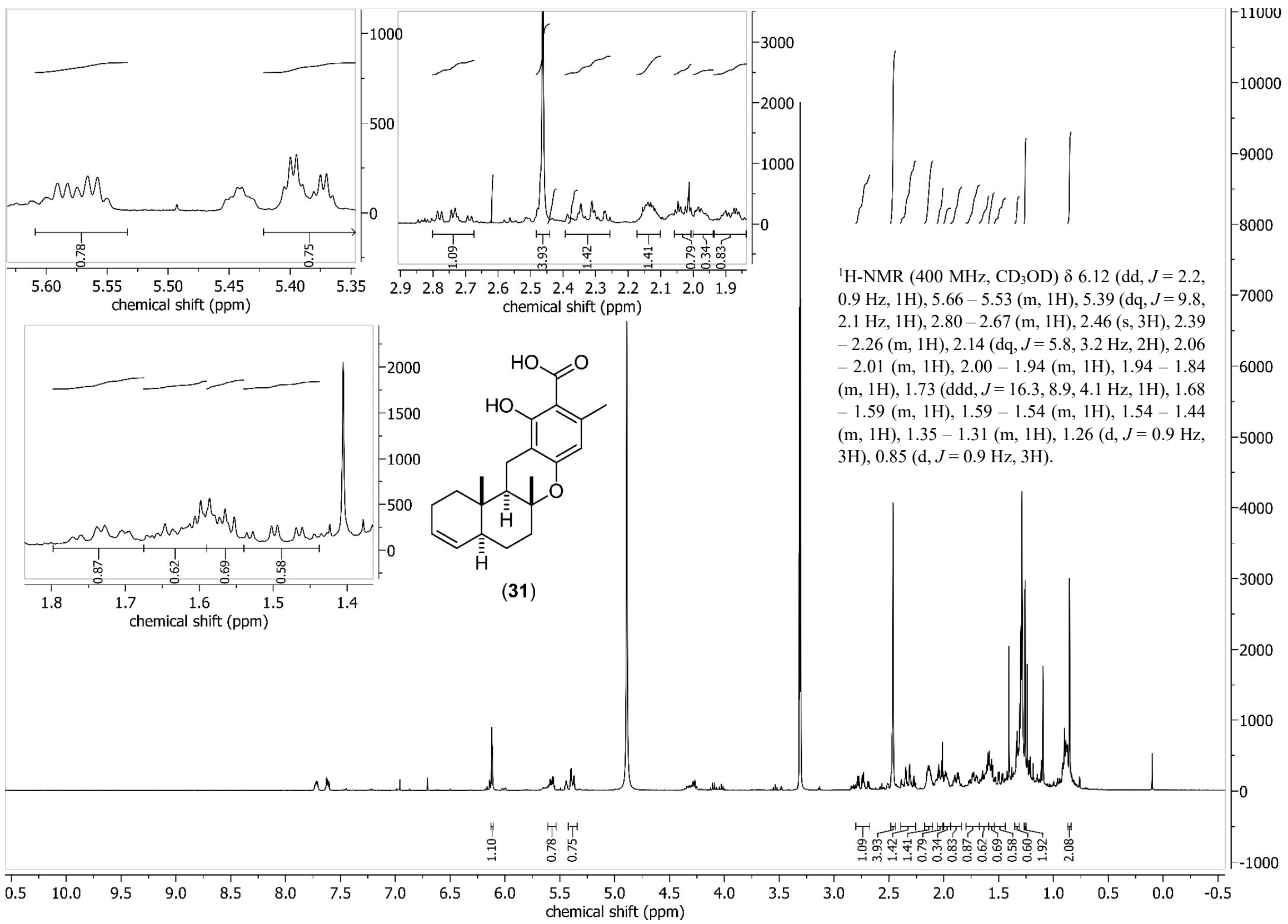

Figure S24: ${ }^{1} \mathrm{H}-\mathrm{NMR}$ spectrum of $( \pm)$-11-Hydroxy-6a,9,12b-trimethyl-1,4a,5,6,6a,12,12a,12b-octahydro-2H-benzo[a]xanthene-10-carboxylic acid (31). 


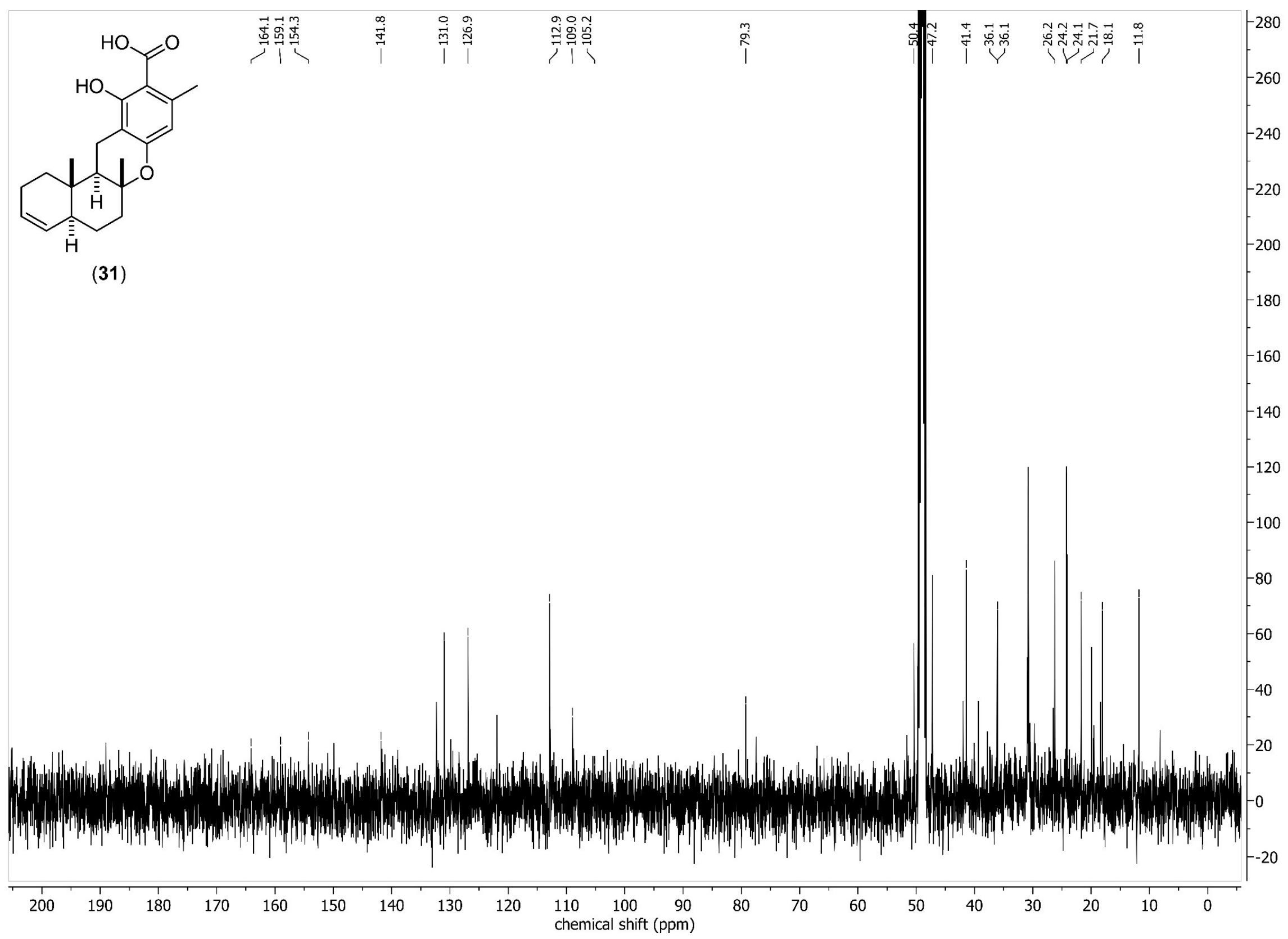

Figure S25: ${ }^{13} \mathrm{C}\left\{{ }^{1} \mathrm{H}\right\}-\mathrm{NMR}\left(101 \mathrm{MHz}, \mathrm{CD}_{3} \mathrm{OD}\right)$ spectrum of $( \pm)$-11-Hydroxy-6a,9,12b-trimethyl-1,4a,5,6,6a,12,12a,12b-octahydro-2H-benzo[a]xanthene-10-carboxylic acid (31). 

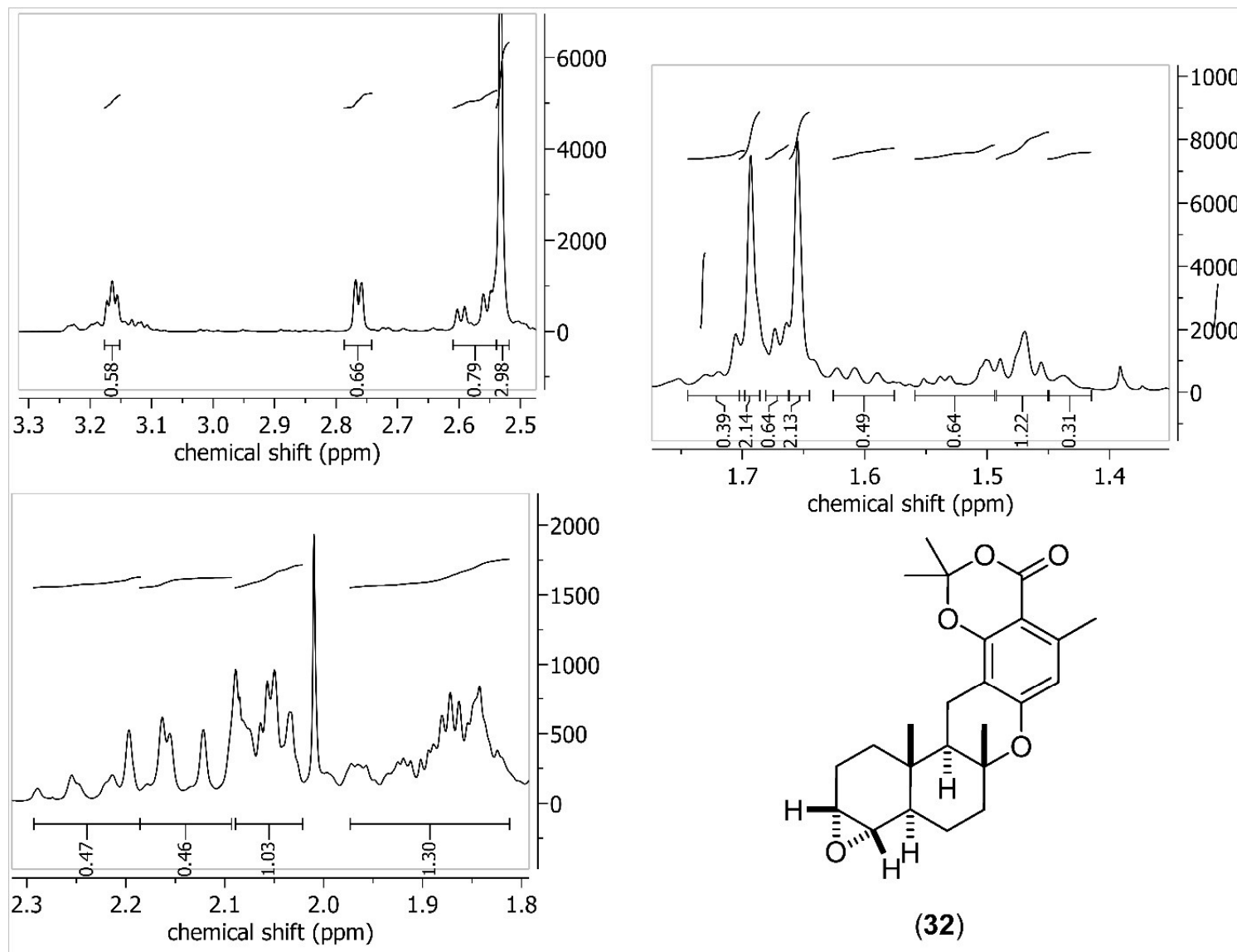

chemical shift (ppm)

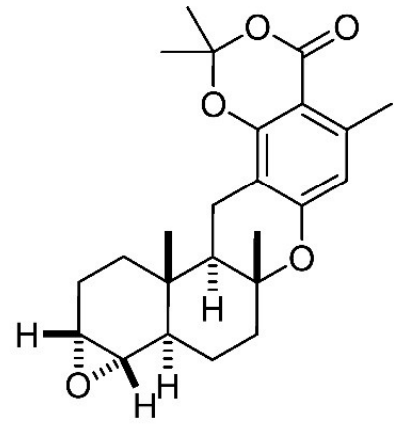

(32) $1 \mathrm{H}), 3.19(\mathrm{t}, J=3.4 \mathrm{~Hz}, 1 \mathrm{H}), 2.79(\mathrm{dt}, J=4.0,0.9-15000$ $\mathrm{Hz}, 1 \mathrm{H}), 2.59$ (dd, $J=17.0,4.8 \mathrm{~Hz}, 1 \mathrm{H}), 2.56$ (s, -14000 $3 \mathrm{H}), 2.27-2.10(\mathrm{~m}, 1 \mathrm{H}), 2.16-2.09(\mathrm{~m}, 1 \mathrm{H}), 2.06$ (ddd, $J=12.8,6.6,2.5 \mathrm{~Hz}, 1 \mathrm{H}), 1.94-1.88(\mathrm{~m}$, 1H) $1.87(\mathrm{td}, J=5.0,4.0,2.3 \mathrm{~Hz}, 1 \mathrm{H}), 1.71(\mathrm{~d}, J=$ $5.6 \mathrm{~Hz}, 1 \mathrm{H}), 1.71(\mathrm{~d}, J=0.7 \mathrm{~Hz}, 3 \mathrm{H}), 1.67(\mathrm{~d}, J=$ $0.7 \mathrm{~Hz}, 3 \mathrm{H}), 1.67-1.60(\mathrm{~m}, 1 \mathrm{H}), 1.57(\mathrm{dd}, J=13.2$, $3.3 \mathrm{~Hz}, 1 \mathrm{H}), 1.53-1.50(\mathrm{~m}, 1 \mathrm{H}) 1.50(\mathrm{~m}, 1 \mathrm{H})$ $1.26(\mathrm{~d}, J=1.0 \mathrm{~Hz}, 3 \mathrm{H}), 1.03-0.95(\mathrm{~m}, 1 \mathrm{H}),$, (d. $J=0.6 \mathrm{~Hz}, 3 \mathrm{H}$ )

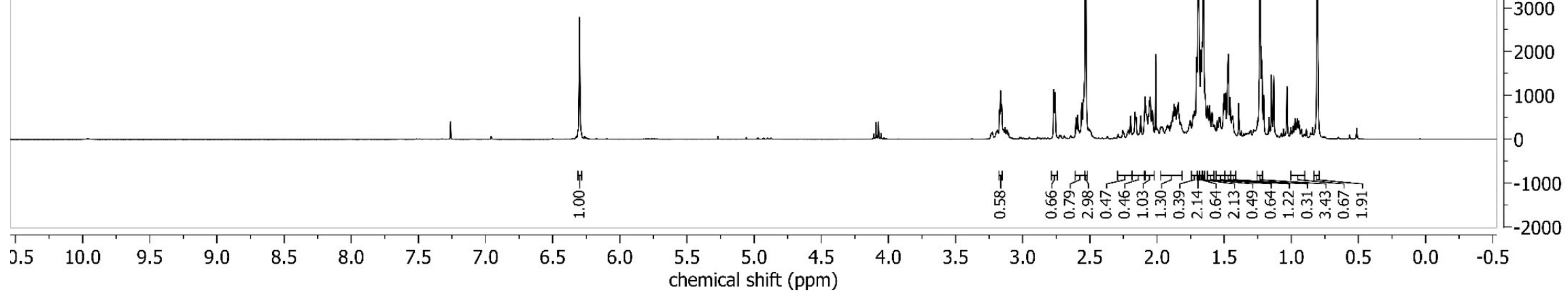

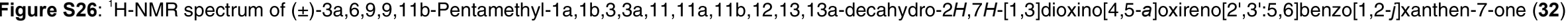




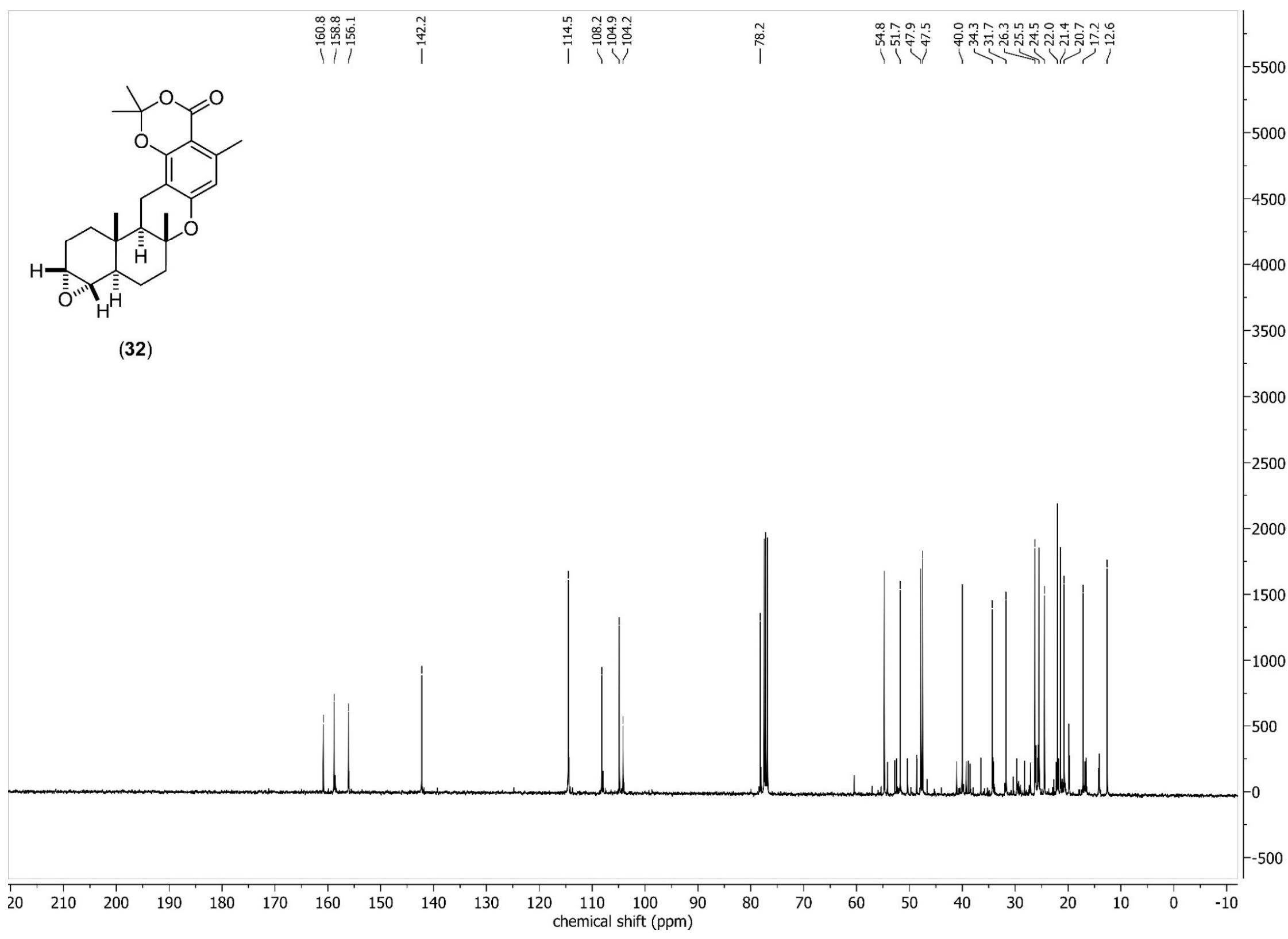

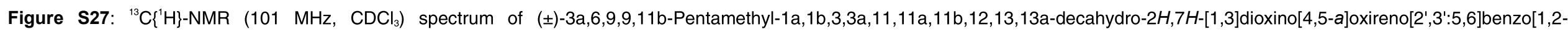
J]xanthen-7-one (32). 


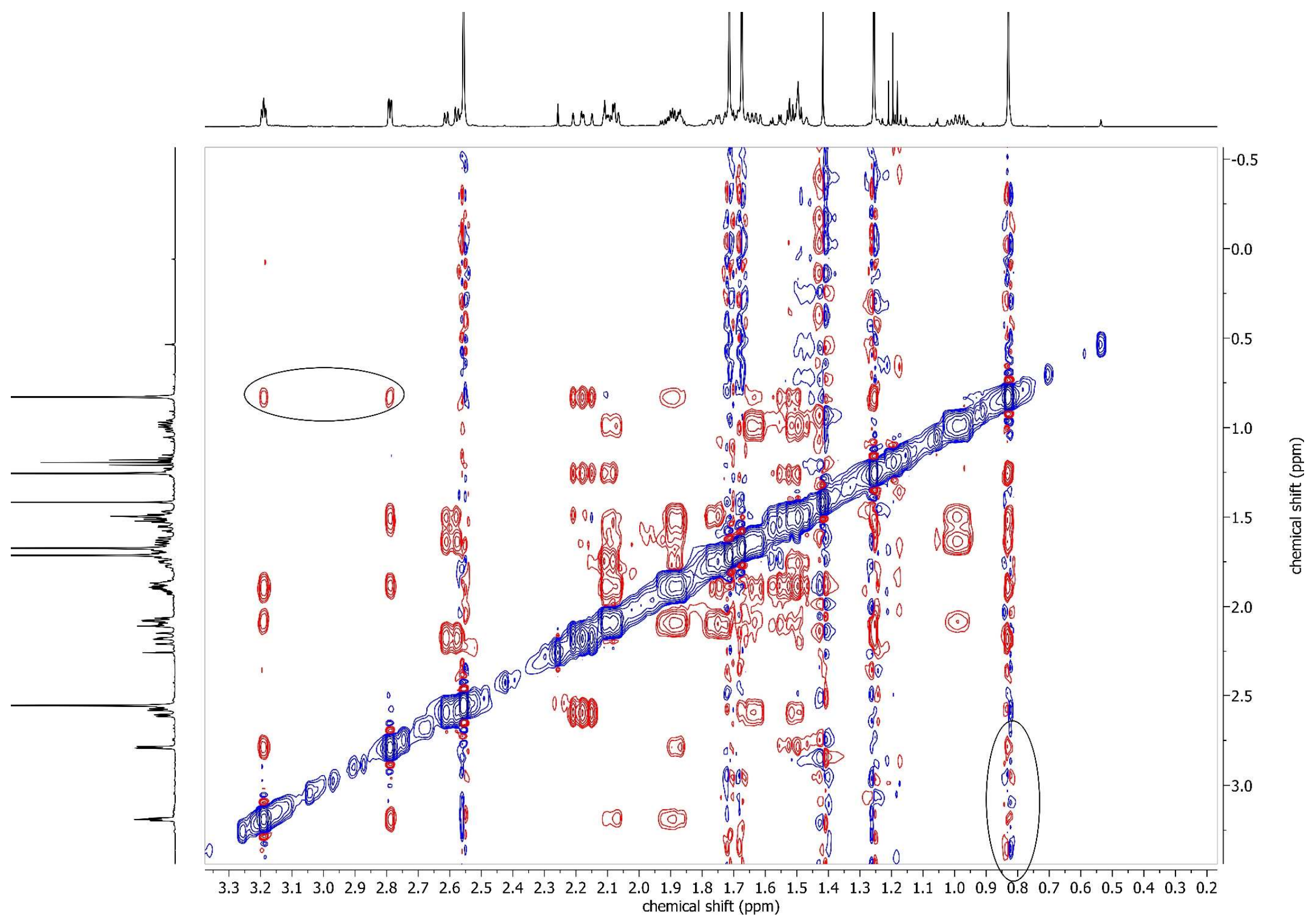

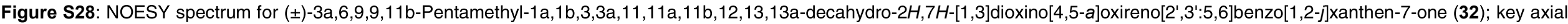
interactions between methyl group and epoxide protons are highlighted. 


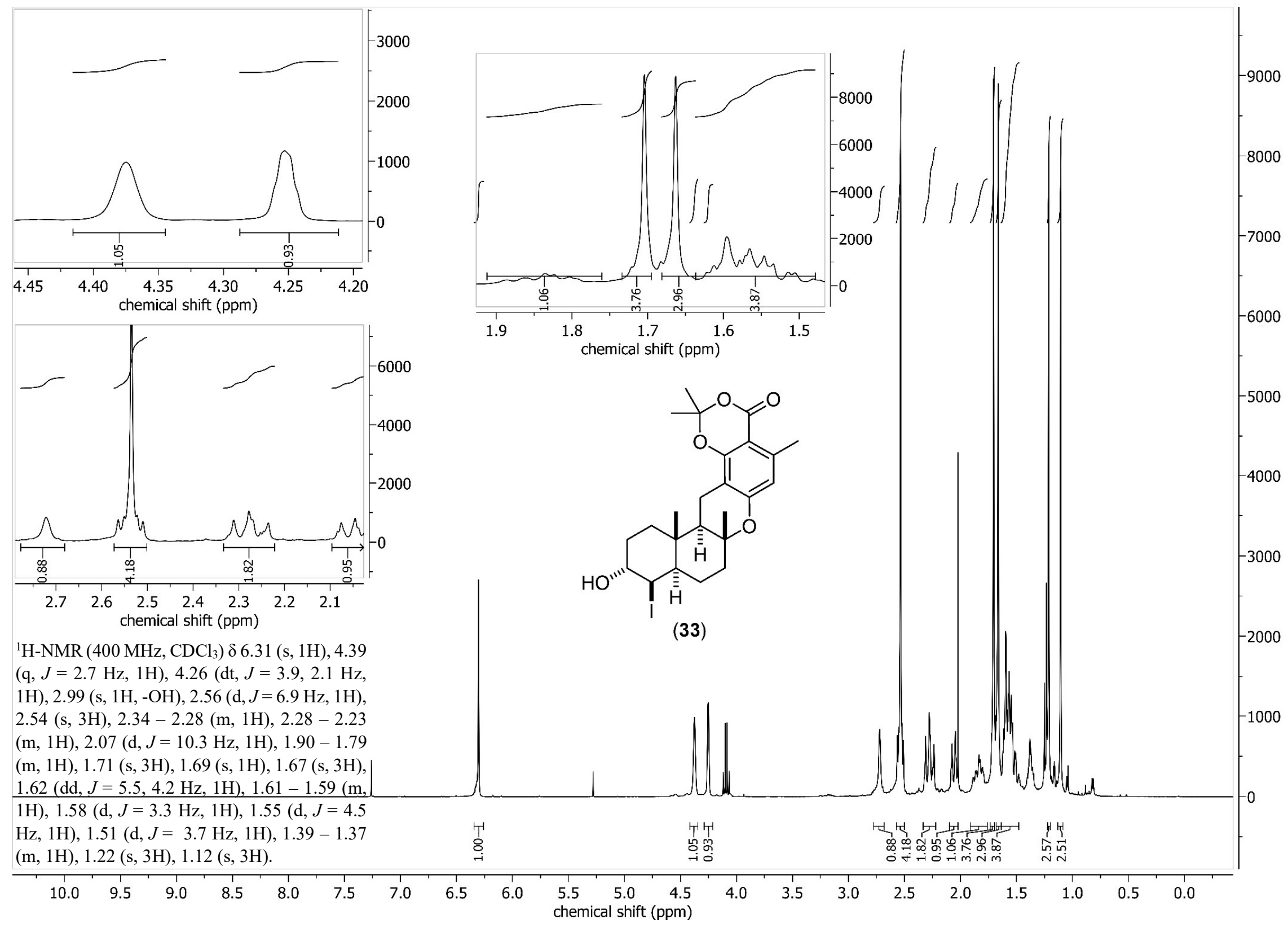

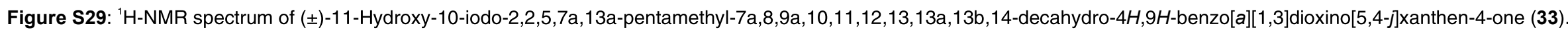




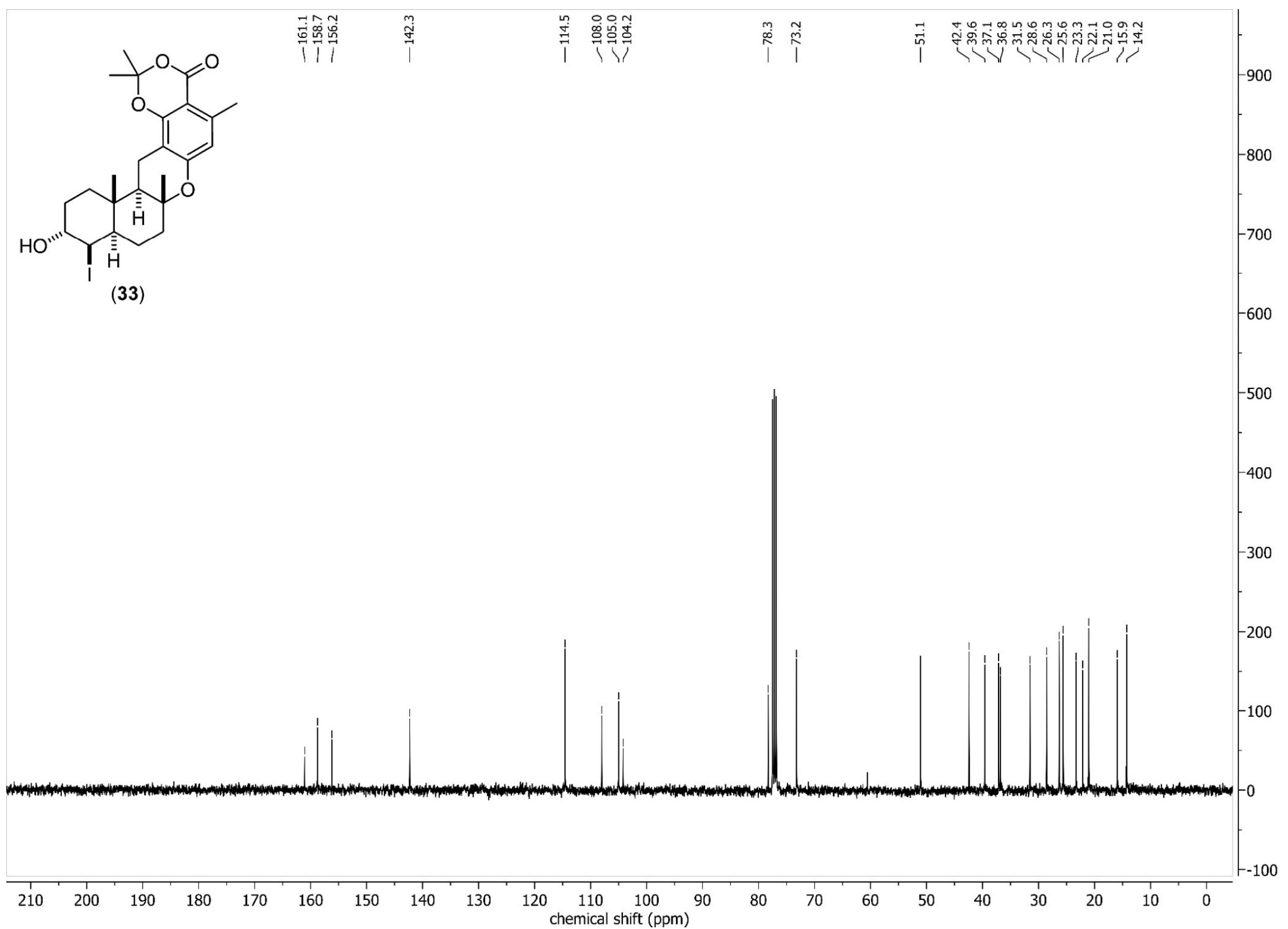

Figure S30: ${ }^{13} \mathrm{C}\left\{{ }^{1} \mathrm{H}\right\}-\mathrm{NMR}\left(101 \mathrm{MHz}, \mathrm{CDCl}_{3}\right)$ spectrum of $( \pm)$-11-Hydroxy-10-iodo-2,2,5,7a,13a-pentamethyl-7a,8,9a,10,11,12,13,13a,13b,14-decahydro-4H,9H-benzo[a][1,3]dioxino[5,4-]xanthen4-one (33). 


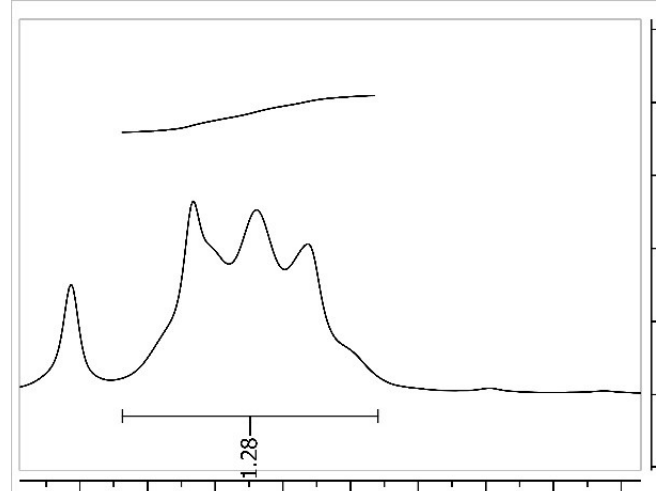

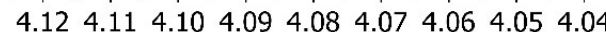
chemical shift (ppm)

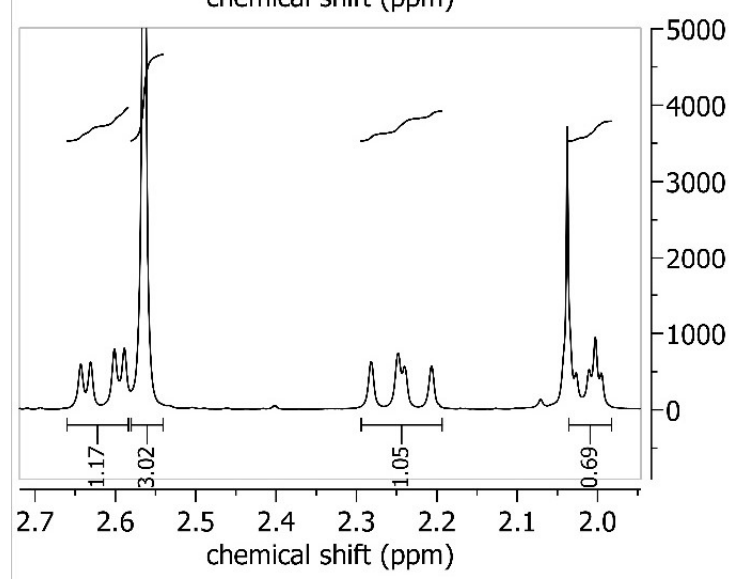

${ }^{1} \mathrm{H}-\mathrm{NMR}\left(400 \mathrm{MHz}, \mathrm{CDCl}_{3}\right) \delta 6.32(\mathrm{~s}, 1 \mathrm{H})$,

4.07 (q, $J=2.9 \mathrm{~Hz}, 1 \mathrm{H}), 2.61$ (dd, $J=16.8,4.9$ $\mathrm{Hz}, 1 \mathrm{H}), 2.55$ (s, 3H), $2.29-2.18(\mathrm{~m}, 1 \mathrm{H})$, $2.00(\mathrm{dt}, J=12.6,3.2 \mathrm{~Hz}, 1 \mathrm{H}), 1.81-1.78(\mathrm{~m}$, $1 \mathrm{H}), 1.77-173(\mathrm{~m}, 1 \mathrm{H}), 1.71(\mathrm{~s}, 3 \mathrm{H}), 1.73(\mathrm{~s}$, $1 \mathrm{H}), 1.67(\mathrm{~s}, 3 \mathrm{H}), 1.64(\mathrm{~d}, J=5.0 \mathrm{~Hz}, 1 \mathrm{H})$, $1.61(\mathrm{~d}, J=4.7 \mathrm{~Hz}, 1 \mathrm{H}), 1.56(\mathrm{dt}, J=7.92 .2 .5$ $\mathrm{Hz}, 1 \mathrm{H}), 1.53-1.46(\mathrm{~m}, 2 \mathrm{H}), 1.47-1.40(\mathrm{~m}$, $1 \mathrm{H}), 1.40-1.30(\mathrm{~m}, 2 \mathrm{H}), 1.21(\mathrm{~s}, 3 \mathrm{H}), 0.82(\mathrm{~s}$, $3 \mathrm{H})$.

$-2000$
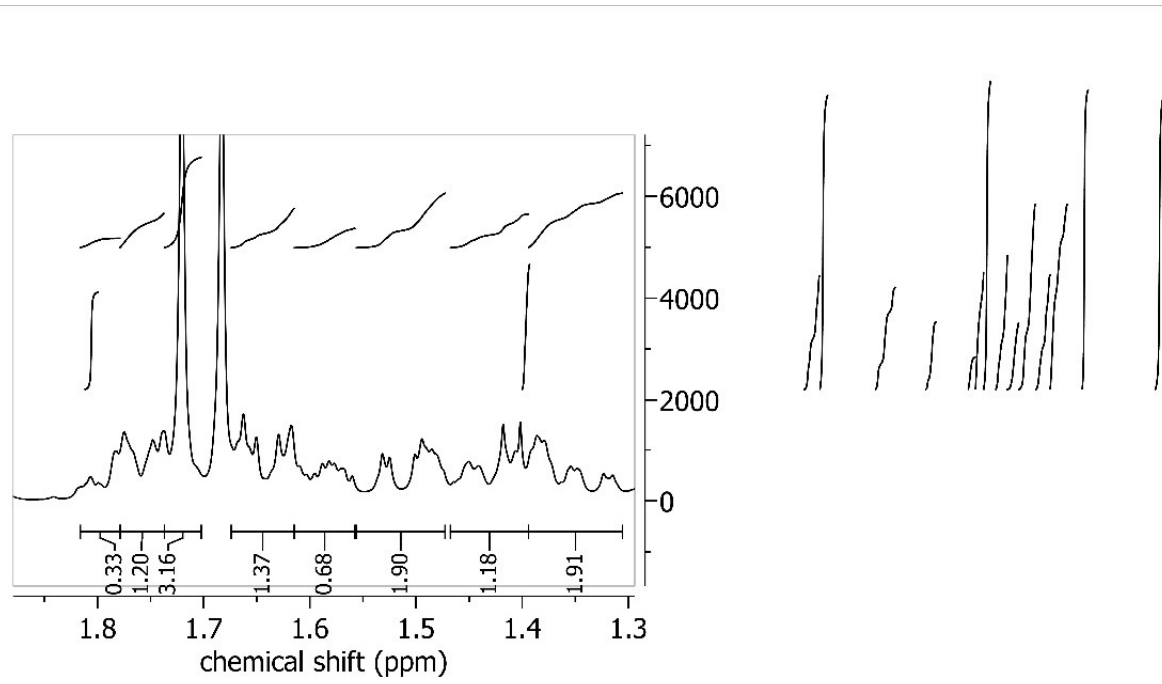

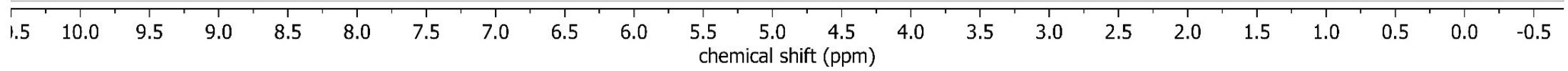

Figure S31: ${ }^{1} \mathrm{H}-N M R$ spectrum of ( \pm )-11-Hydroxy-2,2,5,7a,13a-pentamethyl-7a,8,9a,10,11,12,13,13a,13b,14-decahydro-4H,9H-benzo[a][1,3]dioxino[5,4-j]xanthen-4-one (34). 


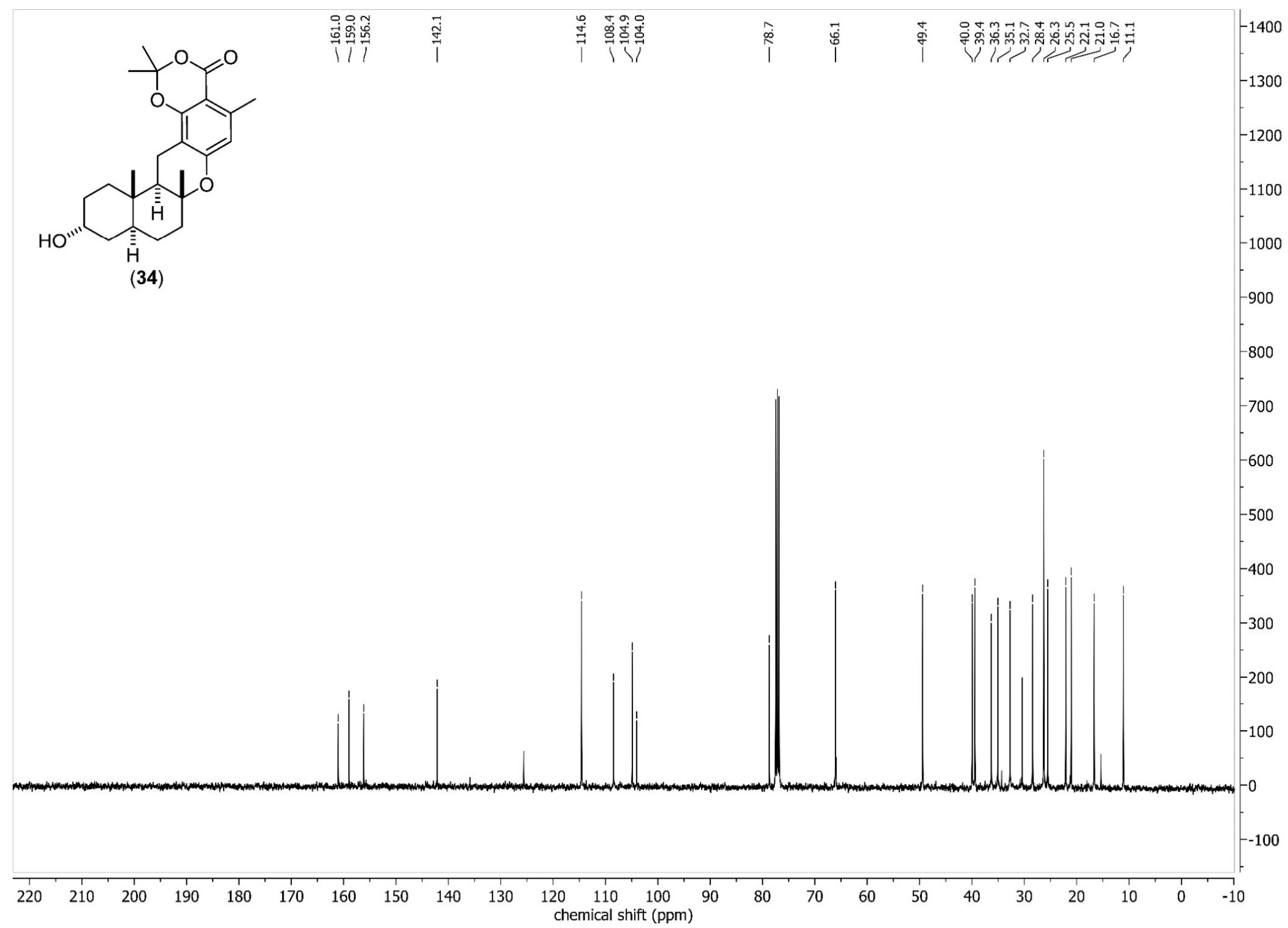

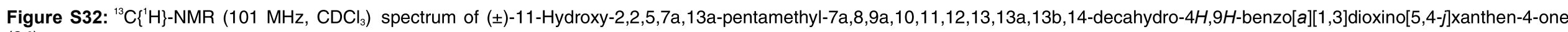
(34). 

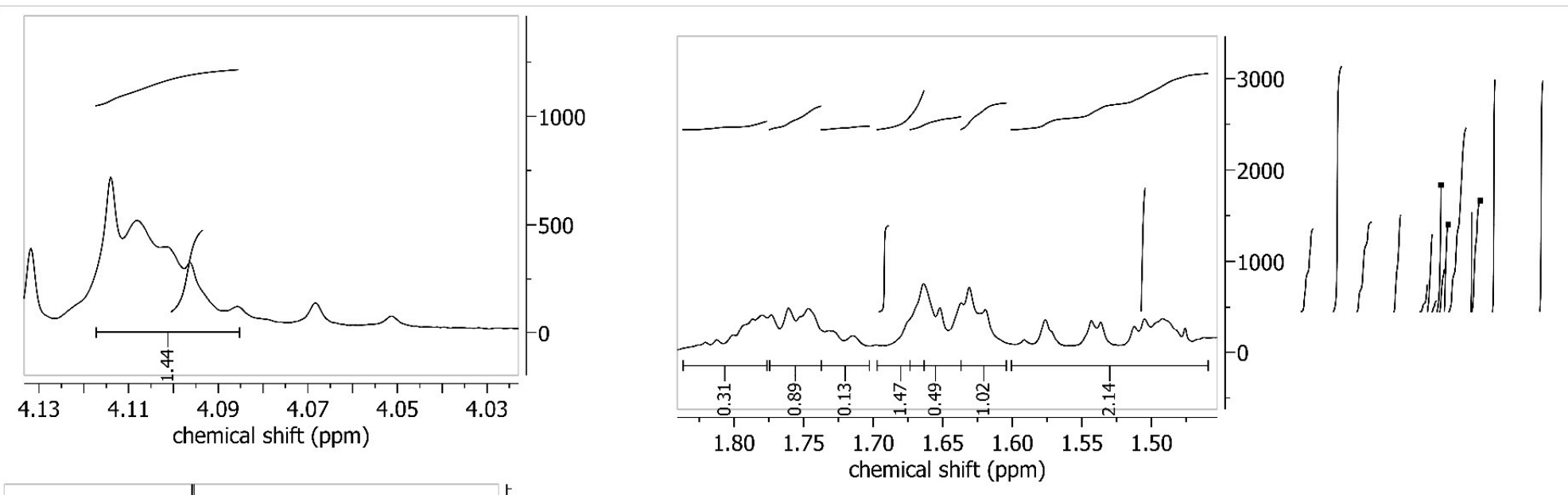

10000
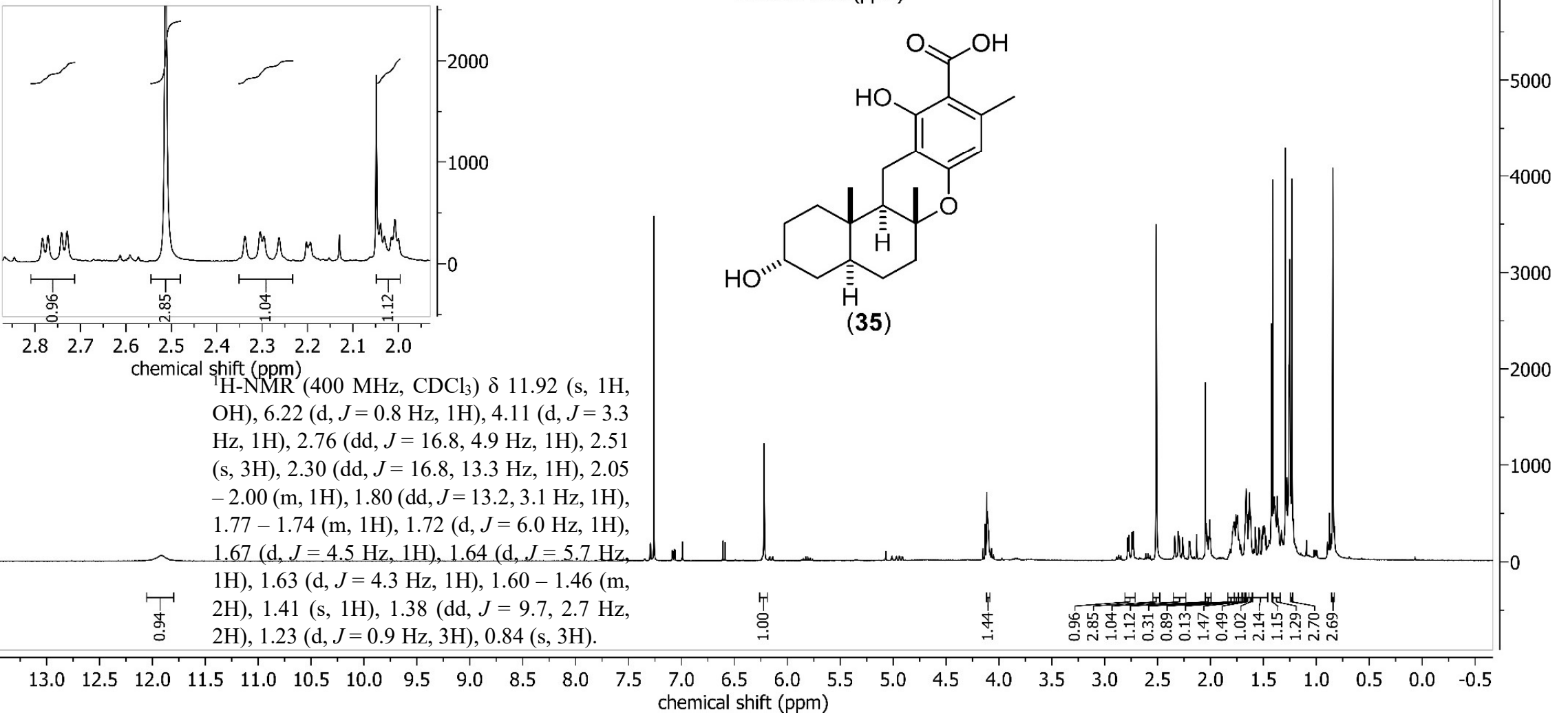

(35)

$\begin{array}{lllllllll}2.8 & 2.7 & 2.6 & 2.5 & 2.4 & 2.3 & 2.2 & 2.1 & 2.0\end{array}$

chemical shift (ppm) $\left(400 \mathrm{MHz}, \mathrm{CDCl}_{3}\right) \delta 11.92(\mathrm{~s}, 1 \mathrm{H}$, $\mathrm{OH}), 6.22(\mathrm{~d}, J=0.8 \mathrm{~Hz}, 1 \mathrm{H}), 4.11(\mathrm{~d}, J=3.3$ $\mathrm{Hz}, 1 \mathrm{H}), 2.76(\mathrm{dd}, J=16.8,4.9 \mathrm{~Hz}, 1 \mathrm{H}), 2.51$ (s, 3H), $2.30(\mathrm{dd}, J=16.8,13.3 \mathrm{~Hz}, 1 \mathrm{H}), 2.05$ $-2.00(\mathrm{~m}, 1 \mathrm{H}), 1.80(\mathrm{dd}, J=13.2,3.1 \mathrm{~Hz}, 1 \mathrm{H})$, $1.77-1.74(\mathrm{~m}, 1 \mathrm{H}), 1.72(\mathrm{~d}, J=6.0 \mathrm{~Hz}, 1 \mathrm{H})$, $1.67(\mathrm{~d}, J=4.5 \mathrm{~Hz}, 1 \mathrm{H}), 1.64(\mathrm{~d}, J=5.7 \mathrm{~Hz}$ $1 \mathrm{H}), 1.63(\mathrm{~d}, J=4.3 \mathrm{~Hz}, 1 \mathrm{H}), 1.60-1.46(\mathrm{~m}$, $2 \mathrm{H}), 1.41(\mathrm{~s}, 1 \mathrm{H}), 1.38(\mathrm{dd}, J=9.7,2.7 \mathrm{~Hz}$, 항 $\quad 2 \mathrm{H}), 1.23(\mathrm{~d}, J=0.9 \mathrm{~Hz}, 3 \mathrm{H}), 0.84(\mathrm{~s}, 3 \mathrm{H})$.

Figure S33: ${ }^{1} \mathrm{H}-\mathrm{NMR}$ of spectrum ( \pm )-3,11-Dihydroxy-6a,9,12b-trimethyl-1,3,4,4a,5,6,6a,12,12a,12b-decahydro-2H-benzo[a]xanthene-10-carboxylic acid (35). 


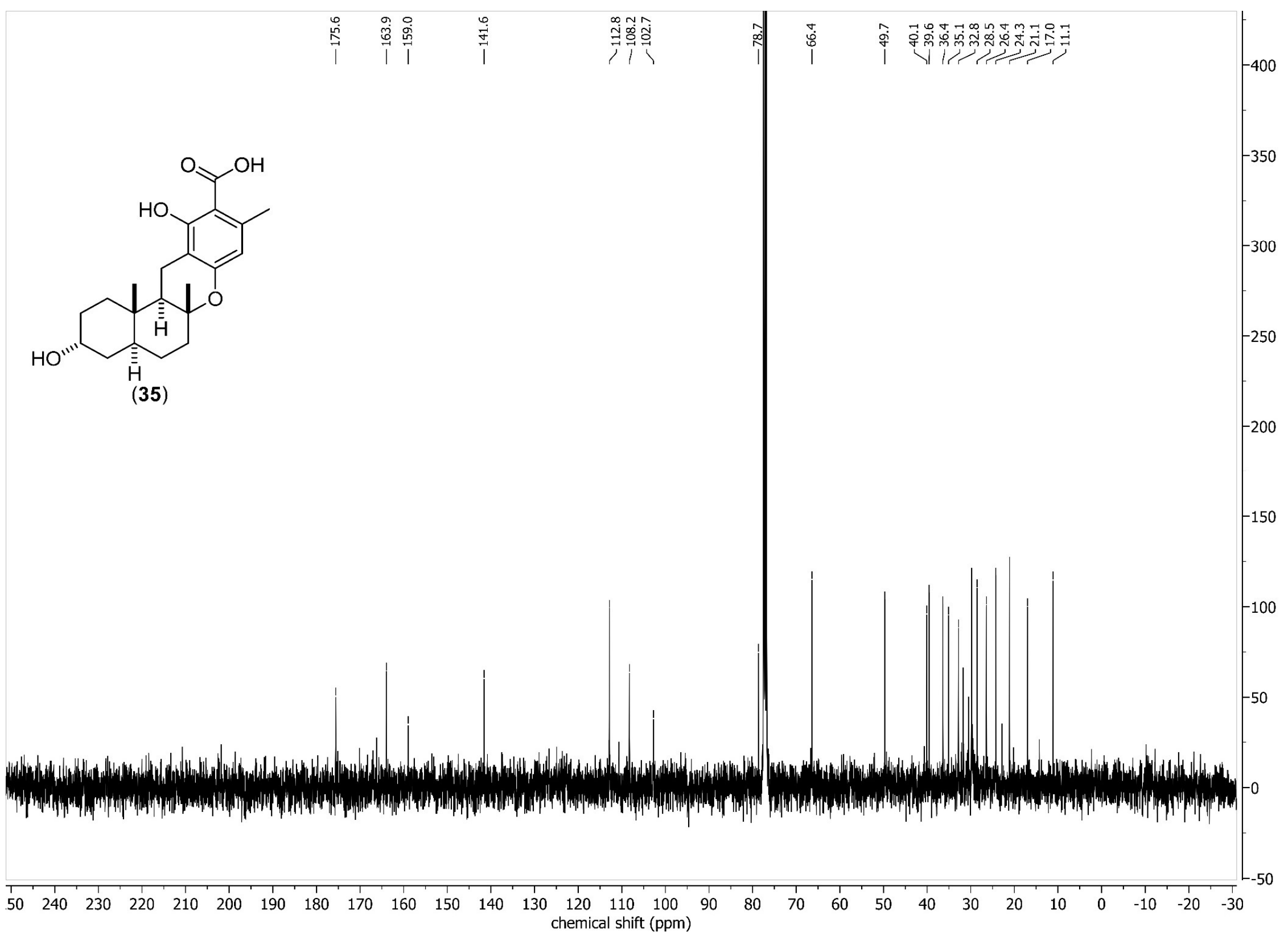

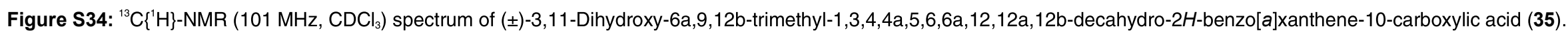




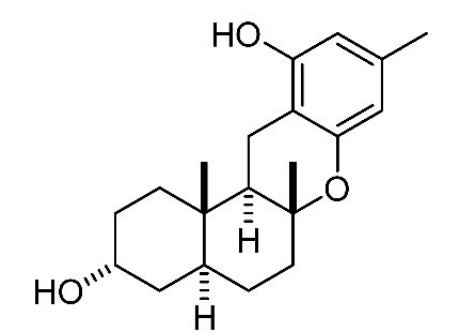

(36)
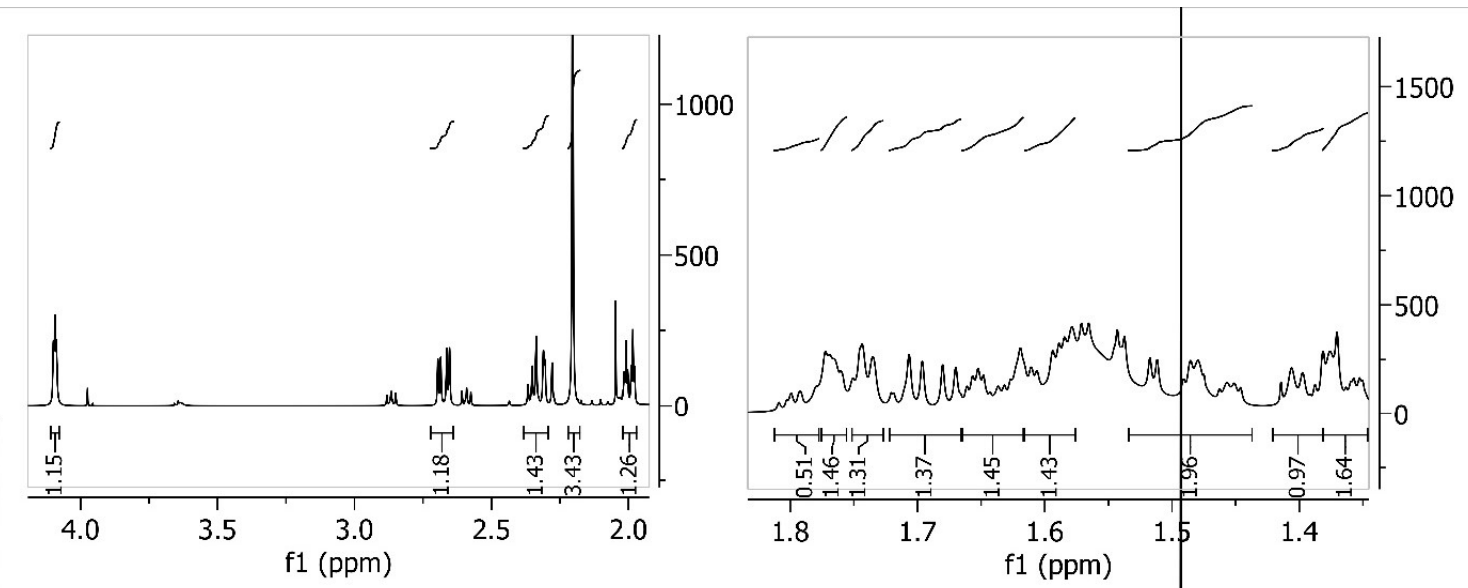

$-7000$

$-6500$

$-6000$

$-5500$

$-5000$

$-4500$

${ }^{1} \mathrm{H}-\mathrm{NMR}\left(500 \mathrm{MHz}, \mathrm{CDCl}_{3}\right) \delta 6.24(\mathrm{~s}, 1 \mathrm{H}), 6.17(\mathrm{~d}, J=1.6 \mathrm{~Hz}, 1 \mathrm{H}), 4.68(\mathrm{~s}, 1 \mathrm{H})$, $4.13-4.06(\mathrm{~m}, 1 \mathrm{H}), 2.67(\mathrm{dd}, J=16.2,5.1 \mathrm{~Hz}, 1 \mathrm{H}), 2.37-2.29(\mathrm{~m}, 1 \mathrm{H}), 2.20(\mathrm{~s}$, $3 \mathrm{H}), 2.00(\mathrm{dt}, J=12.6,3.3 \mathrm{~Hz}, 1 \mathrm{H}), 1.81-1.78(\mathrm{~m}, 1 \mathrm{H}), 1.77(\mathrm{dd}, J=4.4,2.3 \mathrm{~Hz}$,

$1 \mathrm{H}), 1.74(\mathrm{dd}, J=4.7,3.4 \mathrm{~Hz}, 1 \mathrm{H}), 1.72-1.66(\mathrm{~m}, 1 \mathrm{H}), 1.66-1.62(\mathrm{~m}, 1 \mathrm{H}), 1.61-$

$1.58(\mathrm{~m}, 1 \mathrm{H}), 1.53-1.44(\mathrm{~m}, 2 \mathrm{H}), 1.41(\mathrm{t}, J=4.3 \mathrm{~Hz}, 1 \mathrm{H}), 1.39-1.34(\mathrm{~m}, 2 \mathrm{H}), 1.22$

$(\mathrm{d}, J=1.0 \mathrm{~Hz}, 3 \mathrm{H}), 0.85-0.81(\mathrm{~m}, 3 \mathrm{H})$.

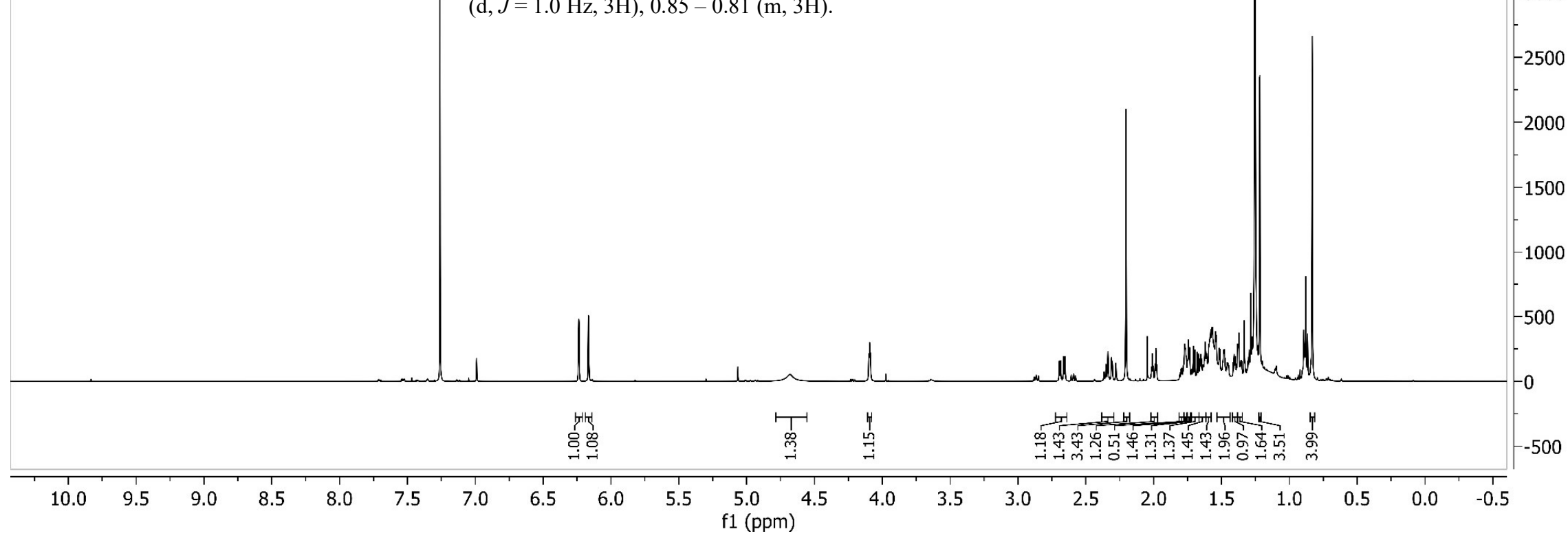

Figure S35: ${ }^{H} \mathrm{H}-\mathrm{NMR}$ of spectrum ( \pm )-6a,9,12b-Trimethyl-1,3,4,4a,5,6,6a,12,12a,12b-decahydro-2H-benzo[a]xanthene-3,11-diol (36). 


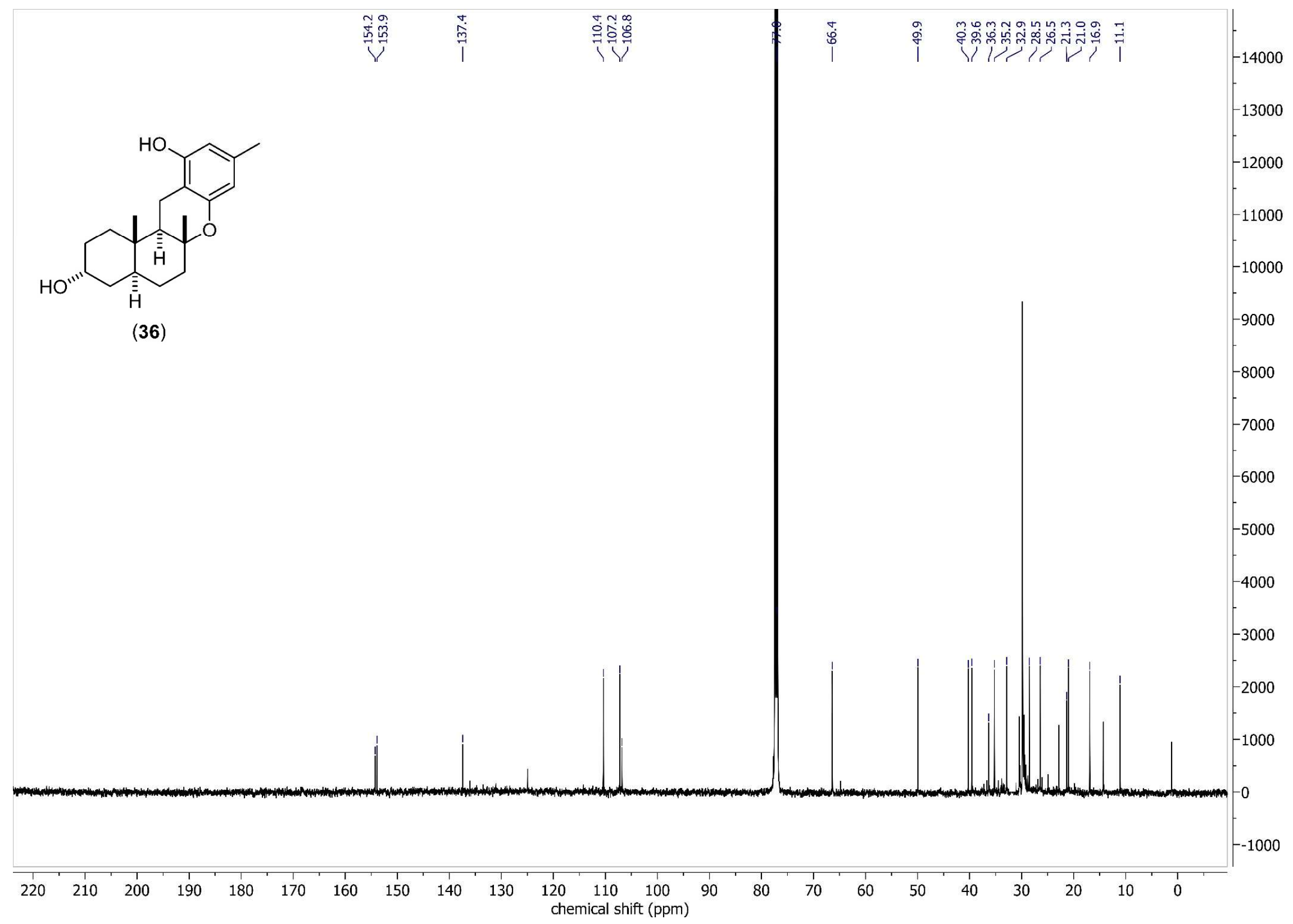

Figure S36: ${ }^{13} \mathrm{C}\left\{{ }^{1} \mathrm{H}\right\}-\mathrm{NMR}(126 \mathrm{MHz}, \mathrm{CDCl})_{3}$ spectrum of ( \pm )-6a,9,12b-Trimethyl-1,3,4,4a,5,6,6a,12,12a,12b-decahydro-2H-benzo[a]xanthene-3,11-diol (36). 

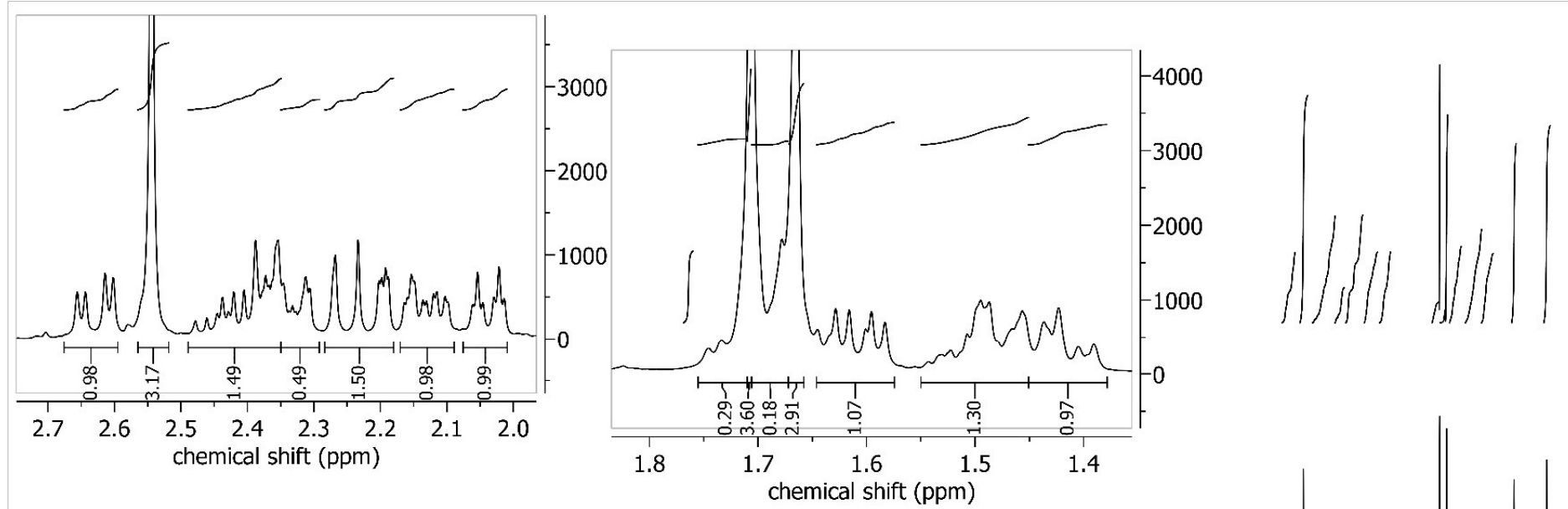

$-13000$

2000

11000

10000

9000

8000

${ }^{1} \mathrm{H}-\mathrm{NMR}\left(400 \mathrm{MHz}, \mathrm{CDCl}_{3}\right) \delta 6.32(\mathrm{~s}, 1 \mathrm{H}), 2.63(\mathrm{dd}, J=16.7,5.0 \mathrm{~Hz}$, $1 \mathrm{H}), 2.54(\mathrm{~s}, 3 \mathrm{H}), 2.49-2.35(\mathrm{~m}, 2 \mathrm{H}), 2.35-2.29(\mathrm{~m}, 1 \mathrm{H}), 2.28-2.18$ $(\mathrm{m}, 2 \mathrm{H}), 2.17-2.09(\mathrm{~m}, 1 \mathrm{H}), 2.04(\mathrm{dt}, J=12.9,3.2 \mathrm{~Hz}, 1 \mathrm{H}), 1.74(\mathrm{~d}, J=$ $5.1 \mathrm{~Hz}, 1 \mathrm{H}), 1.71(\mathrm{~s}, 3 \mathrm{H}, \mathrm{H} 1), 1.68(\mathrm{~s}, 1 \mathrm{H}), 1.67(\mathrm{~s}, 3 \mathrm{H}), 1.65-1.57(\mathrm{~m}$, $1 \mathrm{H}), 1.55-1.45(\mathrm{~m}, 2 \mathrm{H}), 1.45-1.38(\mathrm{~m}, 1 \mathrm{H}), 1.25(\mathrm{~s}, 3 \mathrm{H}), 1.05(\mathrm{~s}, 3 \mathrm{H})$.

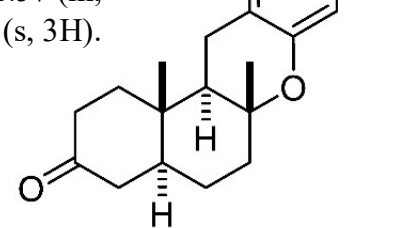

(37)

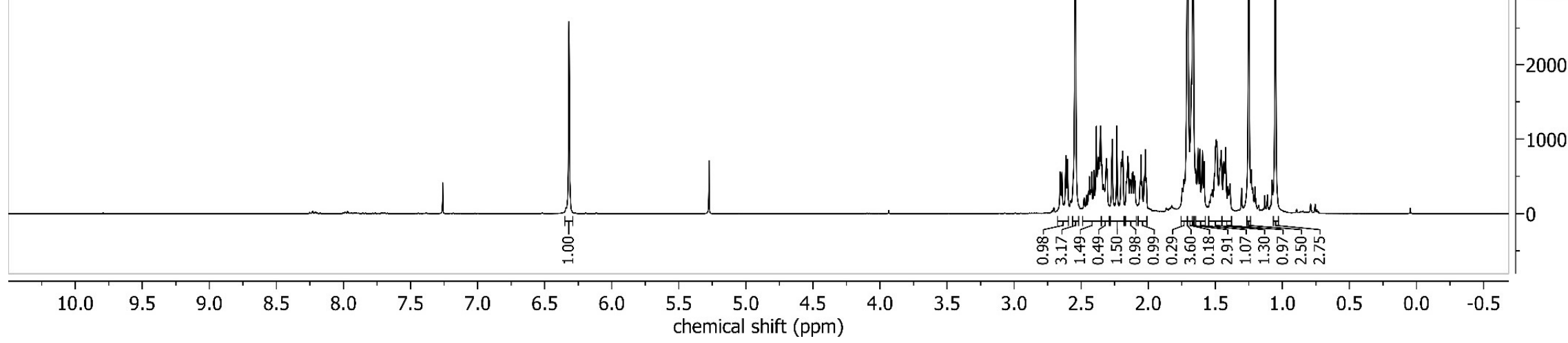

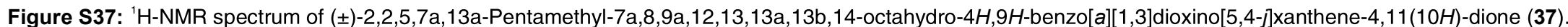




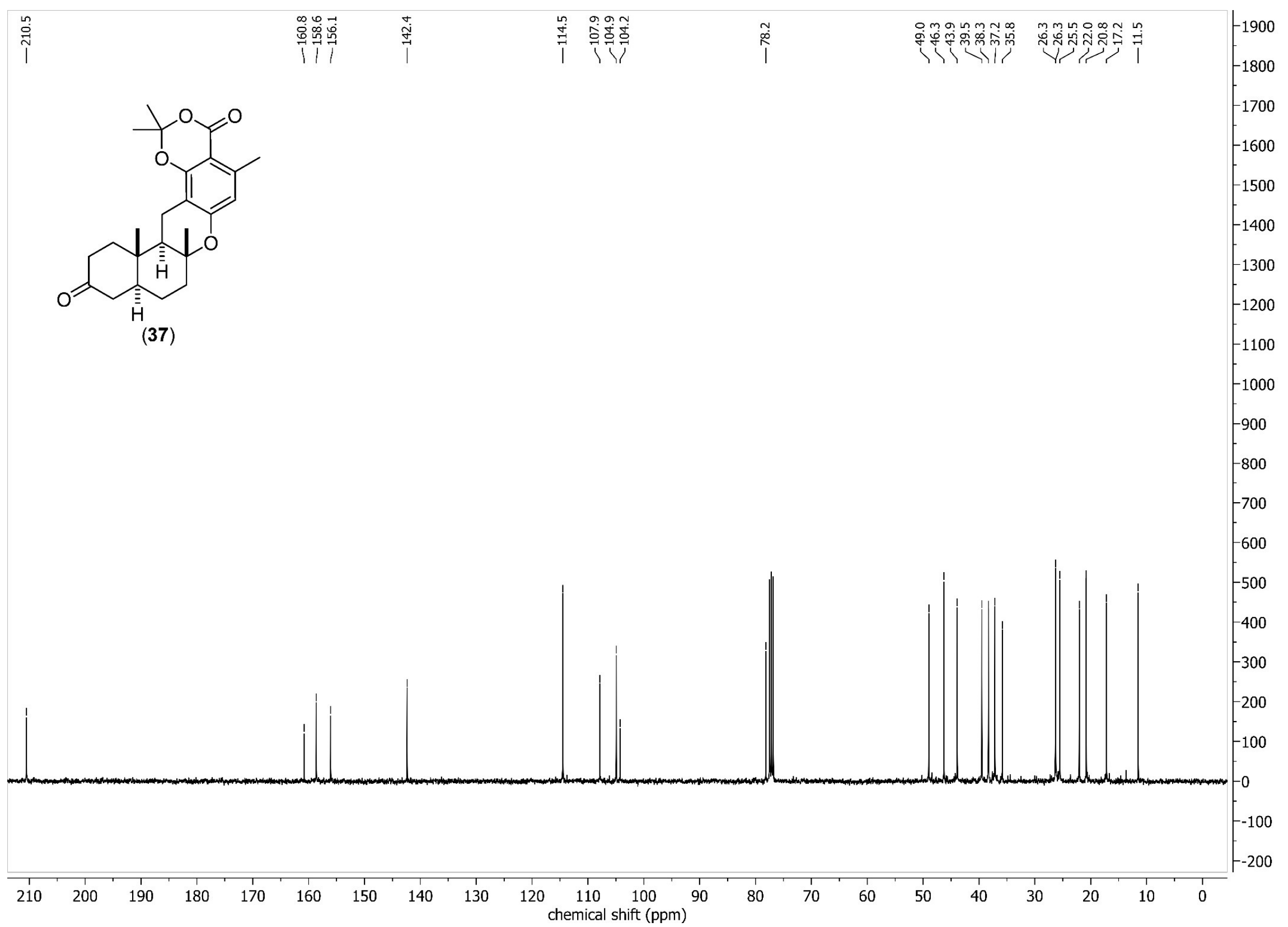

Figure S38: ${ }^{13} \mathrm{C}\{\mathrm{1} H\}-\mathrm{NMR}\left(101 \mathrm{MHz}, \mathrm{CDCl}_{3}\right)$ spectrum of $( \pm)-2,2,5,7 \mathrm{a}, 13 \mathrm{a}$-Pentamethyl-7a,8,9a, 12,13,13a, 13b, 14-octahydro-4H,9H-benzo[a][1,3]dioxino[5,4-]]xanthene-4,11(10H)-dione (37). 

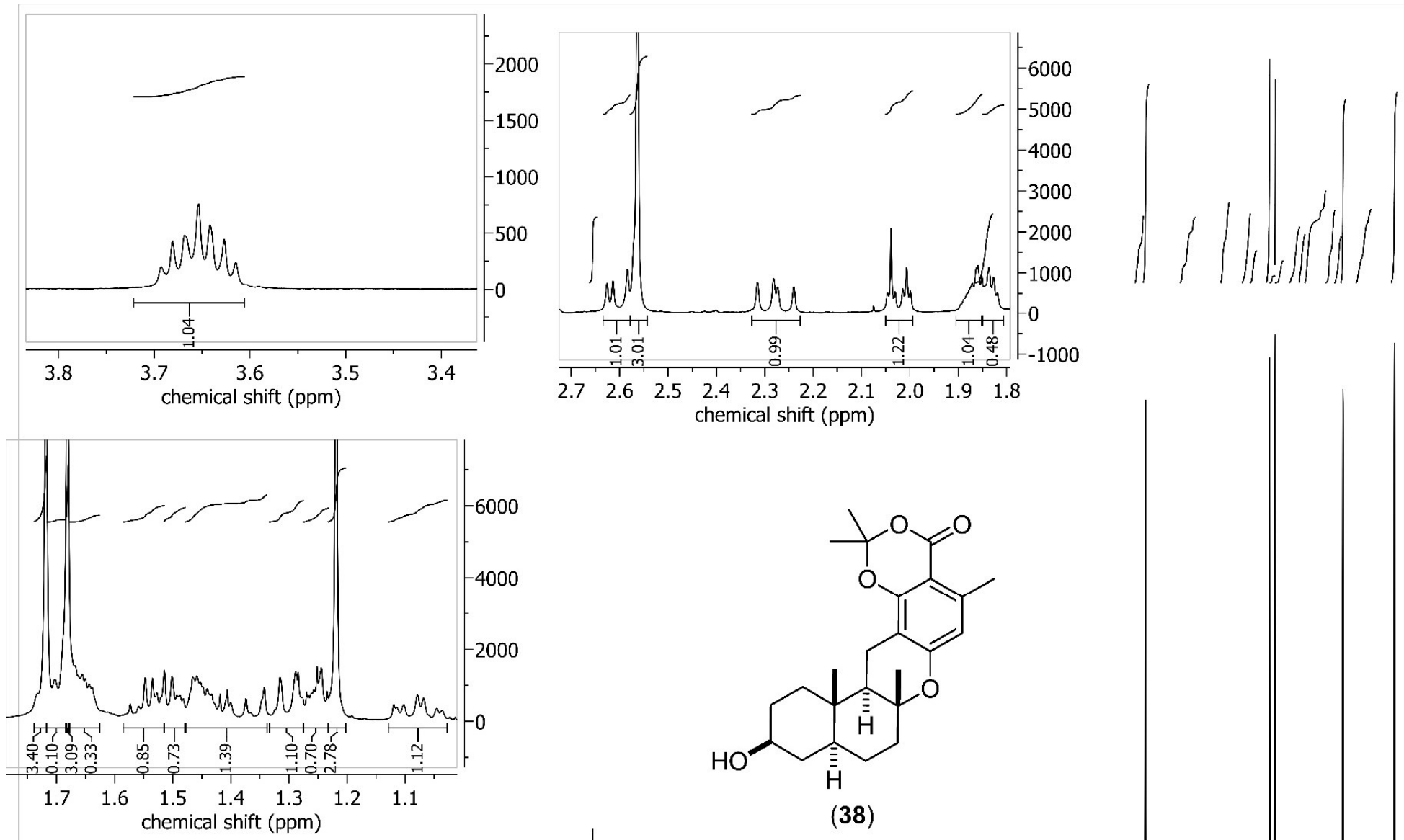

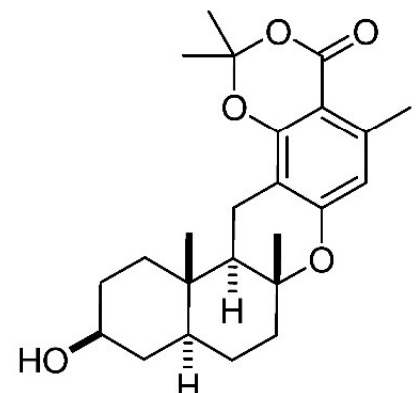

(38)

${ }^{1} \mathrm{H}-\mathrm{NMR}\left(400 \mathrm{MHz}, \mathrm{CDCl}_{3}\right) \delta 6.33(\mathrm{~d}, J=1.0 \mathrm{~Hz}$, $1 \mathrm{H}), 3.65(\mathrm{tt}, J=10.6,4.8 \mathrm{~Hz}, 1 \mathrm{H}), 2.63-2.58(\mathrm{~m}$, $1 \mathrm{H}), 2.56(\mathrm{~s}, 3 \mathrm{H}), 2.28(\mathrm{dd}, J=16.8,13.3 \mathrm{~Hz}, 1 \mathrm{H})$, $2.02(\mathrm{dt}, J=12.6,3.0 \mathrm{~Hz}, 1 \mathrm{H}), 1.90-1.85(\mathrm{~m}, 1 \mathrm{H})$, $1.85-1.81(\mathrm{~m}, 1 \mathrm{H}), 1.72(\mathrm{~s}, 3 \mathrm{H}), 1.70(\mathrm{~d}, J=1.0$ $\mathrm{Hz}, 1 \mathrm{H}), 1.68$ (s, 3H), 1.65 (dd, $J=4.8,2.2 \mathrm{~Hz}$, $1 \mathrm{H}), 1.59-1.51(\mathrm{~m}, 1 \mathrm{H}), 1.51-1.46(\mathrm{~m}, 1 \mathrm{H}), 1.48$ - $1.34(\mathrm{~m}, 2 \mathrm{H}), 1.30(\mathrm{dd}, J=11.3,1.5 \mathrm{~Hz}, 1 \mathrm{H})$, $1.27-1.23(\mathrm{~m}, 1 \mathrm{H}), 1.22(\mathrm{~d}, J=0.9 \mathrm{~Hz}, 3 \mathrm{H}), 1.13$

$-1.03(\mathrm{~m}, 1 \mathrm{H}) 087(\mathrm{~s}, 3 \mathrm{H})$ $\begin{array}{llllll}10.0 & 9.5 & 9.0 & 8.5 & 8.0 & 7.5\end{array}$

Figure S39: ${ }^{1} \mathrm{H}-\mathrm{NMR}$ spectrum of $( \pm)-11$-Hydroxy-2,2,5,7a,13a-pentamethyl-7a,8,9a,10,11,12,13,13a,13b,14-decahydro-4H,9H-benzo[a][1,3]dioxino[5,4-j]xanthen-4-one (38). 


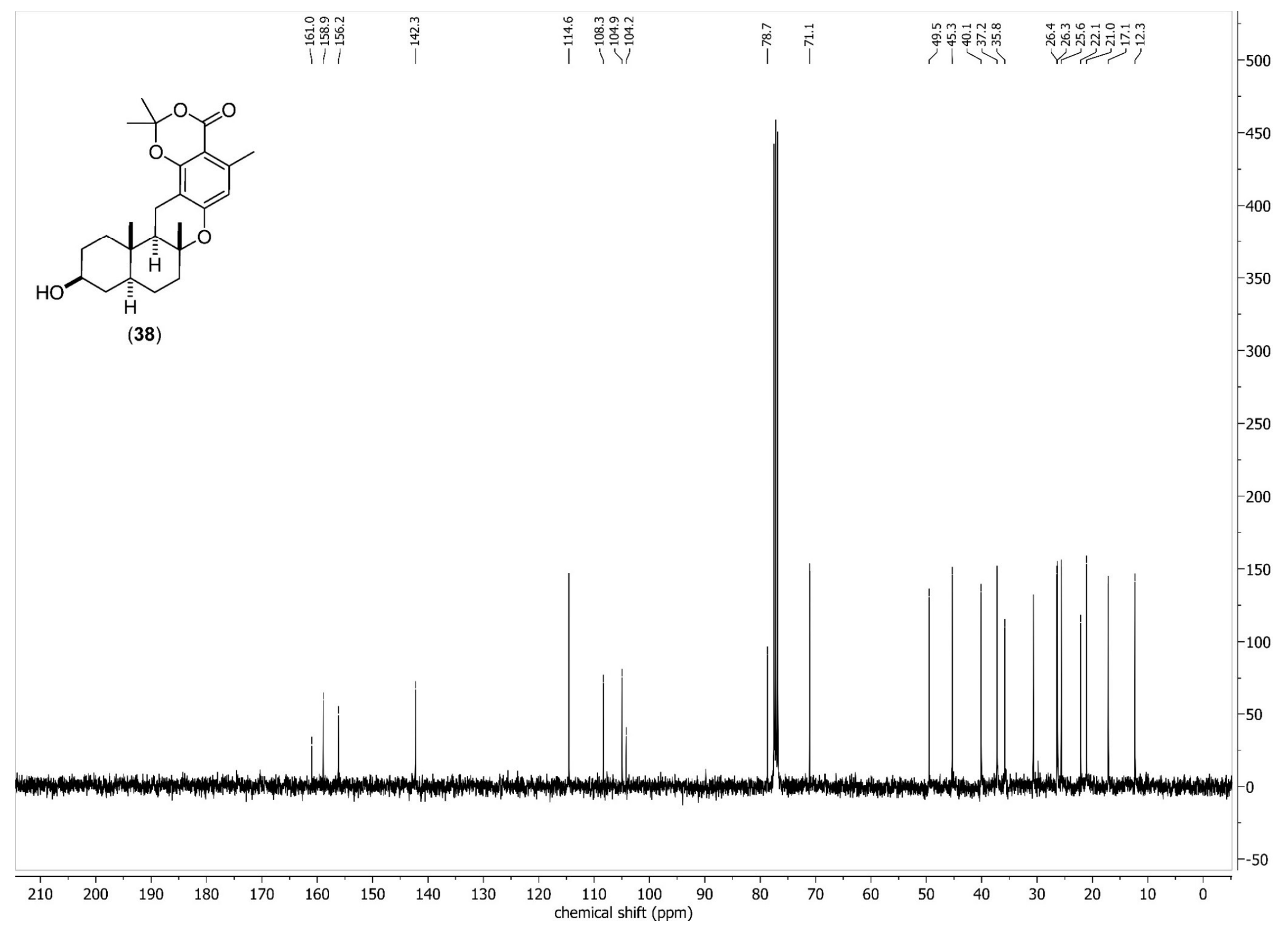

Figure S40: ${ }^{13} \mathrm{C}\left\{\mathrm{H} H\right.$-NMR $\left(101 \mathrm{MHz}, \mathrm{CDCl}_{3}\right)$ spectrum of ( \pm )-11-Hydroxy-2,2,5,7a,13a-pentamethyl-7a,8,9a,10,11,12,13,13a, 13b,14-decahydro-4H,9H-benzo[a][1,3]dioxino[5,4-j]xanthen-4-one (38). 


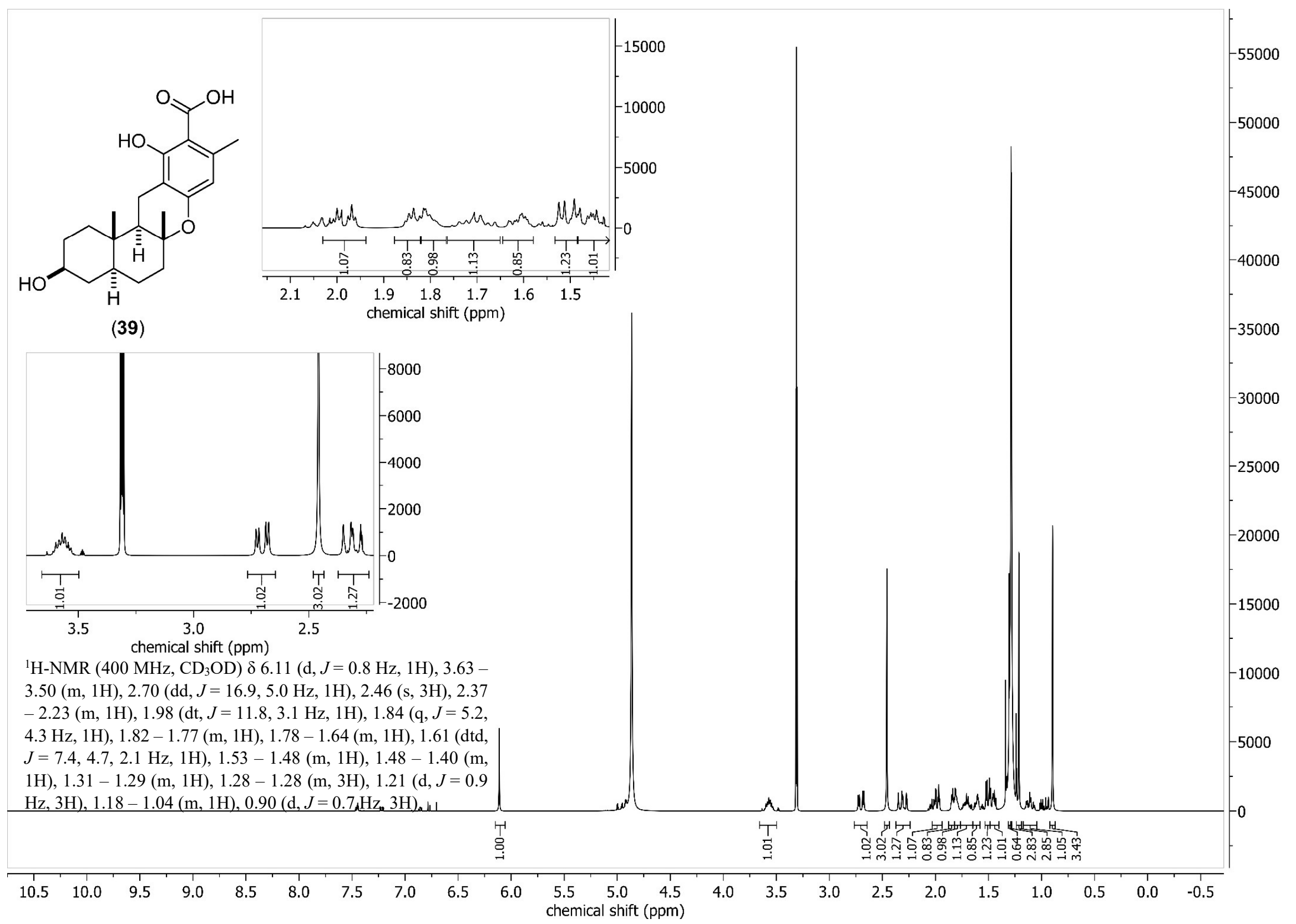

Figure S41: 'H-NMR spectrum of ( \pm )-3,11-Dihydroxy-6a,9,12b-trimethyl-1,3,4,4a,5,6,6a,12,12a,12b-decahydro-2H-benzo[a]xanthene-10-carboxylic acid (39). 


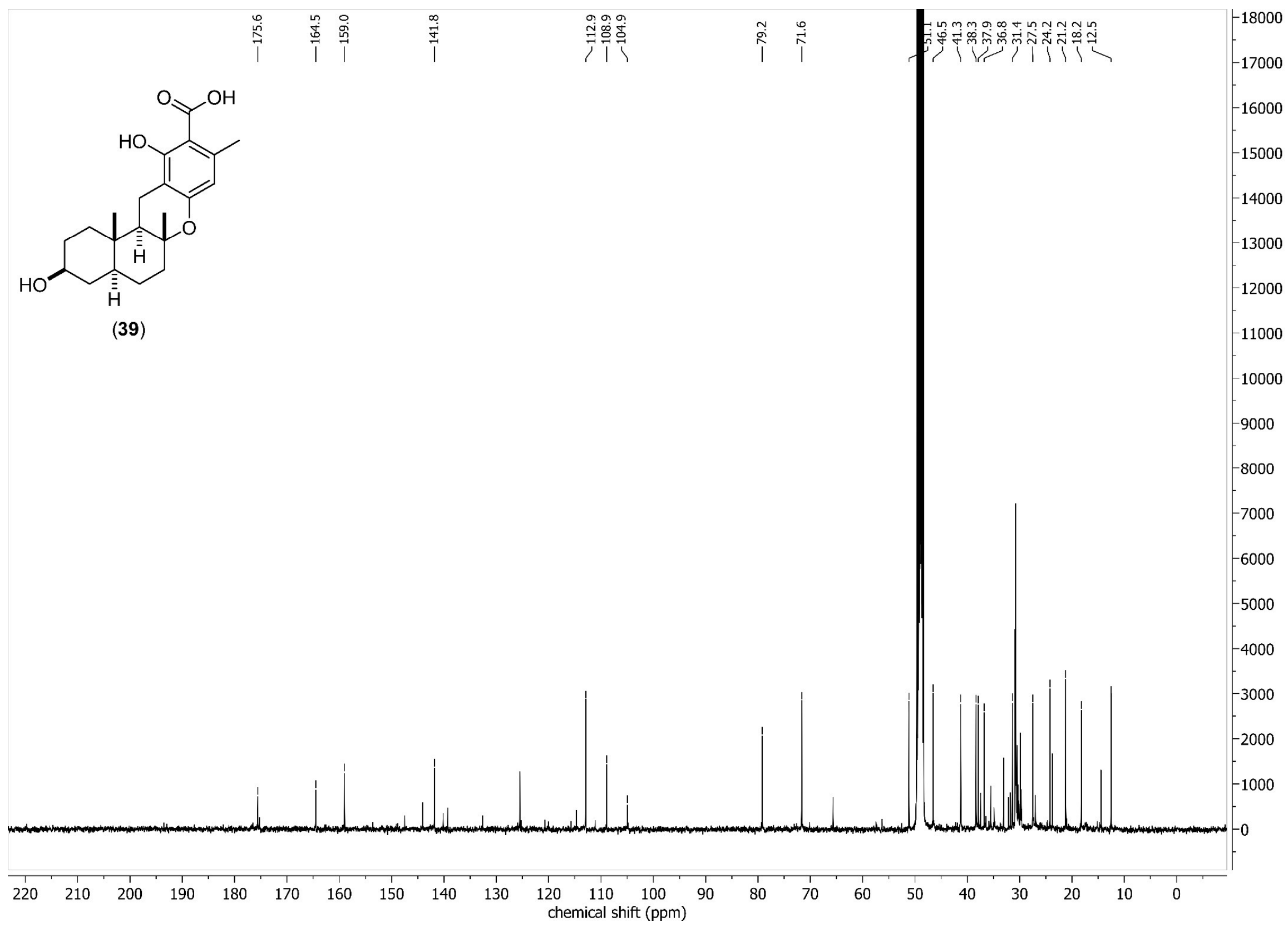

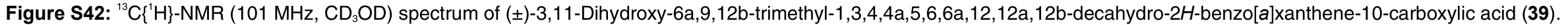




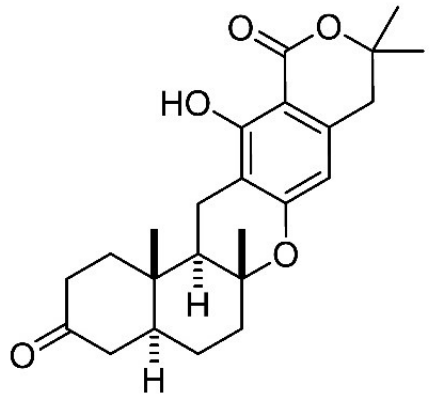

(40)

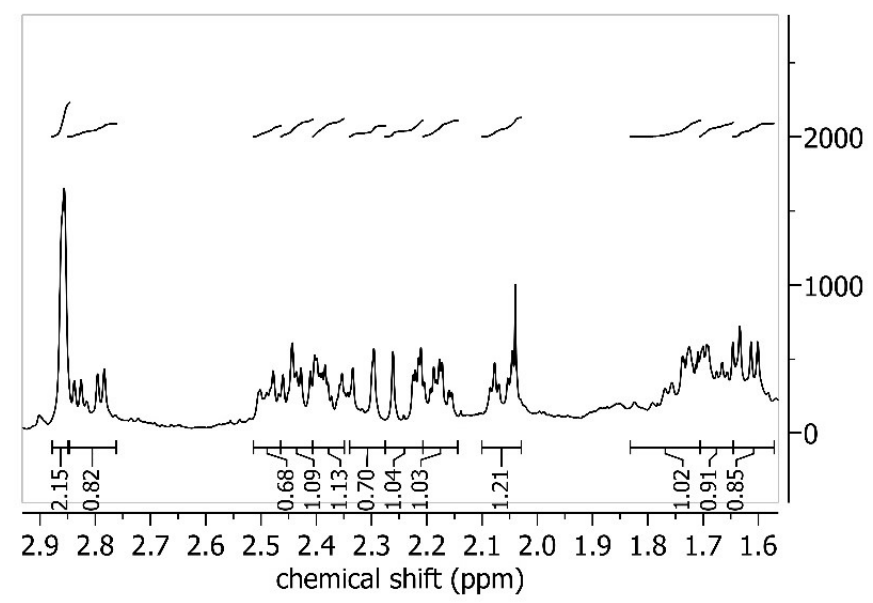

${ }^{1} \mathrm{H}-\mathrm{NMR}\left(400 \mathrm{MHz}, \mathrm{CDCl}_{3}\right) \delta 11.63(\mathrm{~s}, 1 \mathrm{H}), 6.16-6.09(\mathrm{~m}, 1 \mathrm{H}), 2.86(\mathrm{~d}$, $J=2.4 \mathrm{~Hz}, 2 \mathrm{H}), 2.81(\mathrm{dd}, J=16.8,4.9 \mathrm{~Hz}, 1 \mathrm{H}), 2.49(\mathrm{dt}, J=8.6,2.8 \mathrm{~Hz}$, $1 \mathrm{H}), 2.46-2.41(\mathrm{~m}, 1 \mathrm{H}), 2.41-2.35(\mathrm{~m}, 1 \mathrm{H}), 2.32(\mathrm{dd}, J=15.5,1.6 \mathrm{~Hz}$, $1 \mathrm{H}), 2.27-2.21(\mathrm{~m}, 1 \mathrm{H}), 2.21-2.14(\mathrm{~m}, 1 \mathrm{H}), 2.10-2.03(\mathrm{~m}, 1 \mathrm{H}), 1.83-$ $1.70(\mathrm{~m}, 1 \mathrm{H}), 1.71-1.65(\mathrm{~m}, 1 \mathrm{H}), 1.65-1.57(\mathrm{~m}, 1 \mathrm{H}), 1.51(\mathrm{ddt}, J=7.3$, 4.0, $2.4 \mathrm{~Hz}, 1 \mathrm{H}), 1.46(\mathrm{~s}, 3 \mathrm{H}), 1.45(\mathrm{~d}, J=4.1 \mathrm{~Hz}, 5 \mathrm{H}), 1.30(\mathrm{~d}, J=0.9 \mathrm{~Hz}$, $3 \mathrm{H}), 1.08$ (s, 3H).

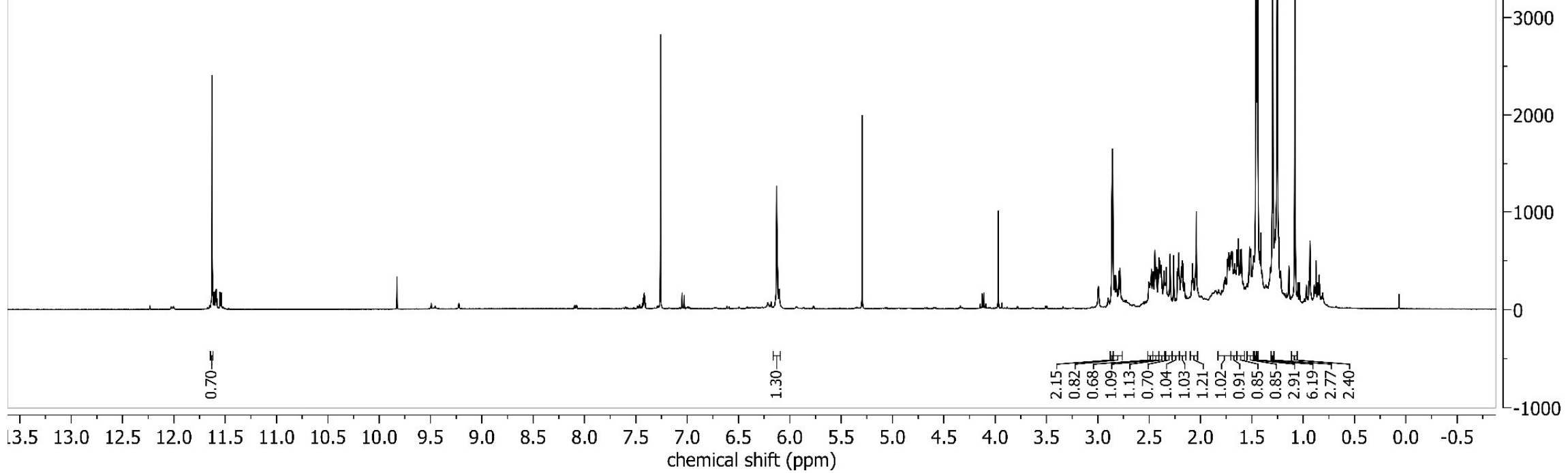

Figure S43: ${ }^{1} \mathrm{H}-\mathrm{NMR}$ spectrum of $( \pm)$-13-Hydroxy-6a,10,10,14b-tetramethyl-1,4,4a,5,6,6a,9,14,14a,14b-decahydro-2 $\mathrm{H}, 10 \mathrm{H}$-benzo[a]pyrano[4,3-I]xanthene-3,12-dione $(40)$. 


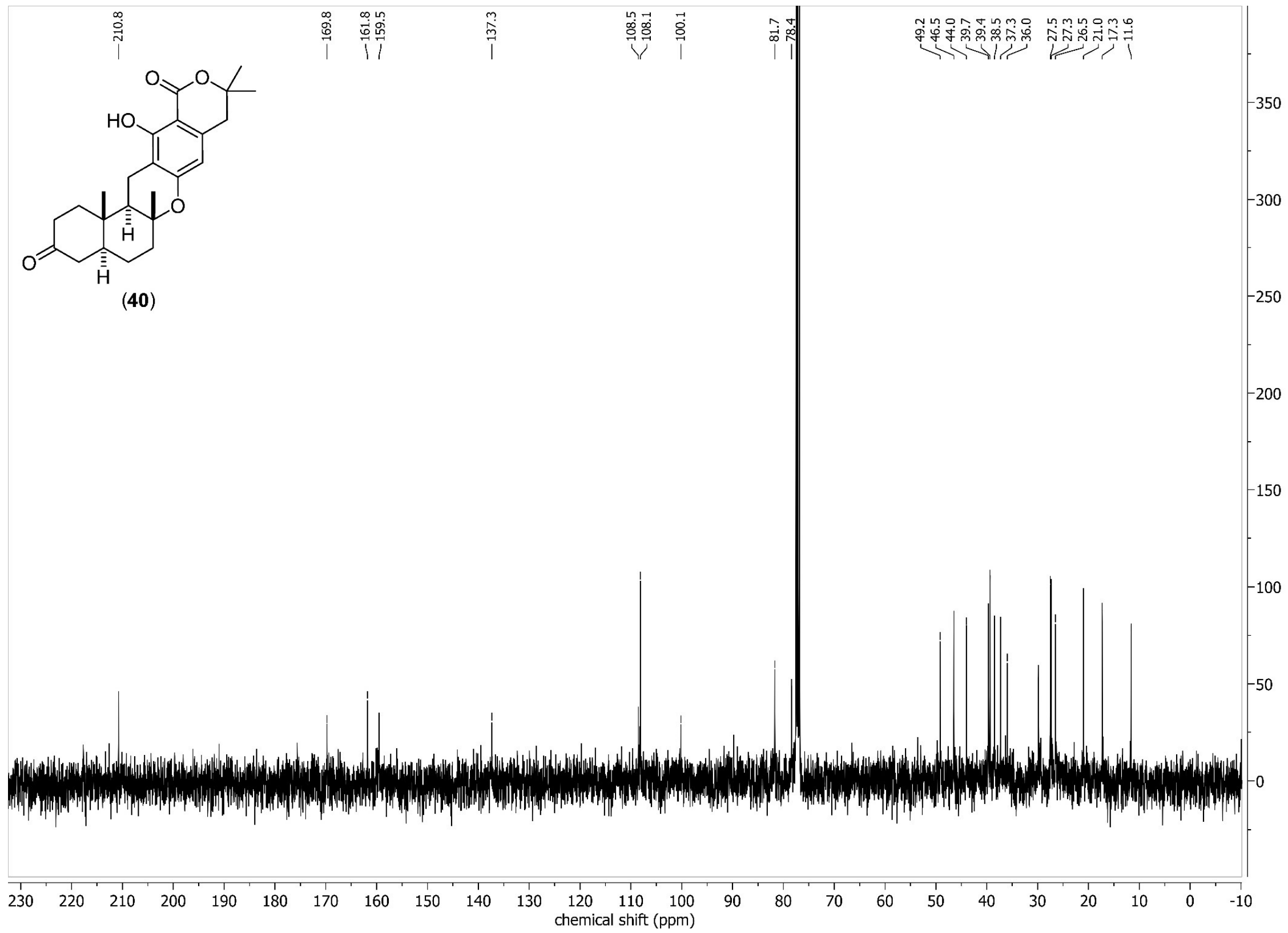

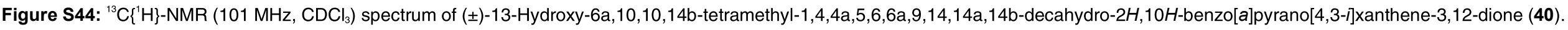

S46 

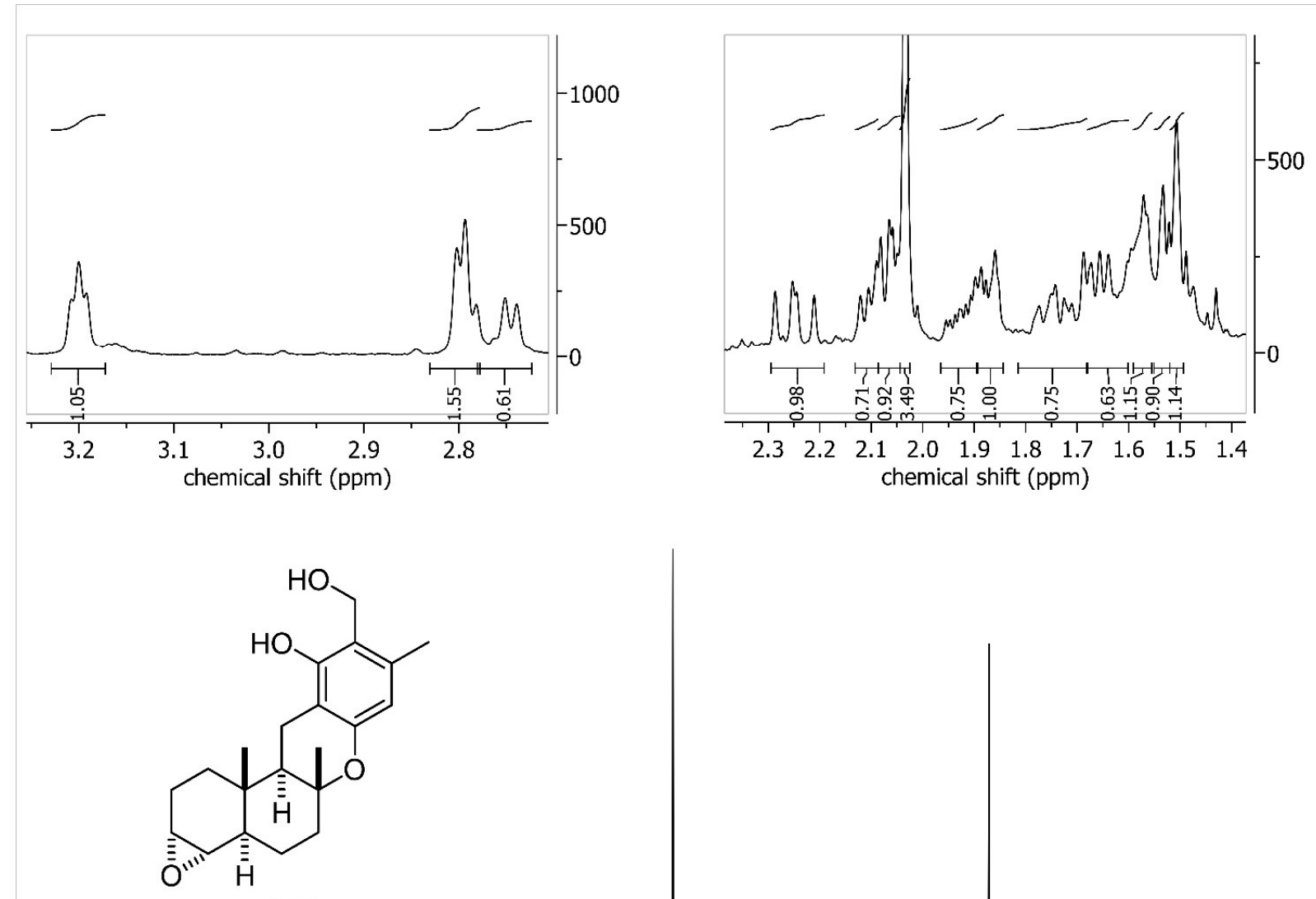

(41)

${ }^{1} \mathrm{H}-\mathrm{NMR}\left(400 \mathrm{MHz}, \mathrm{CDCl}_{3}\right) \delta 6.31(\mathrm{~s}, 1 \mathrm{H}), 4.94(\mathrm{~s}$, $2 \mathrm{H}), 3.20(\mathrm{t}, J=3.5 \mathrm{~Hz}, 1 \mathrm{H}), 2.80(\mathrm{~d}, J=3.8 \mathrm{~Hz}, 1 \mathrm{H})$, $2.75(\mathrm{~d}, J=5.0 \mathrm{~Hz}, 1 \mathrm{H}), 2.25$ (dd, $J=16.9,13.3 \mathrm{~Hz}$, 1H), $2.13-2.09(\mathrm{~m}, 1 \mathrm{H}), 2.09-2.04(\mathrm{~m}, 1 \mathrm{H}), 2.03(\mathrm{~s}$, $3 \mathrm{H}), 1.97-1.90(\mathrm{~m}, 1 \mathrm{H}), 1.89-1.84(\mathrm{~m}, 1 \mathrm{H}), 1.81-$ $1.68(\mathrm{~m}, 1 \mathrm{H}), 1.68-1.60(\mathrm{~m}, 1 \mathrm{H}), 1.57(\mathrm{~d}, J=3.5 \mathrm{~Hz}$, $1 \mathrm{H}), 1.53(\mathrm{t}, J=3.4 \mathrm{~Hz}, 1 \mathrm{H}), 1.50(\mathrm{~d}, J=2.8 \mathrm{~Hz}, 1 \mathrm{H})$,

$1.25(\mathrm{~s}, 3 \mathrm{H}), 0.99(\mathrm{~s}, 1 \mathrm{H}), 0.84(\mathrm{~s}, 3 \mathrm{H})$

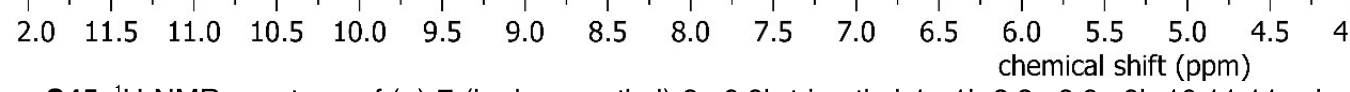

Figure S45: ' ${ }^{H}$-NMR spectrum of ( \pm )-7-(hydroxymethyl)-3a,6,9b-trimethyl-1a,1b,3,3a,9,9a,9b,10,11,11a-decahydro-2H-oxireno[2',3':3,4]benzo[1,2-a]xanthen-8-ol (41).

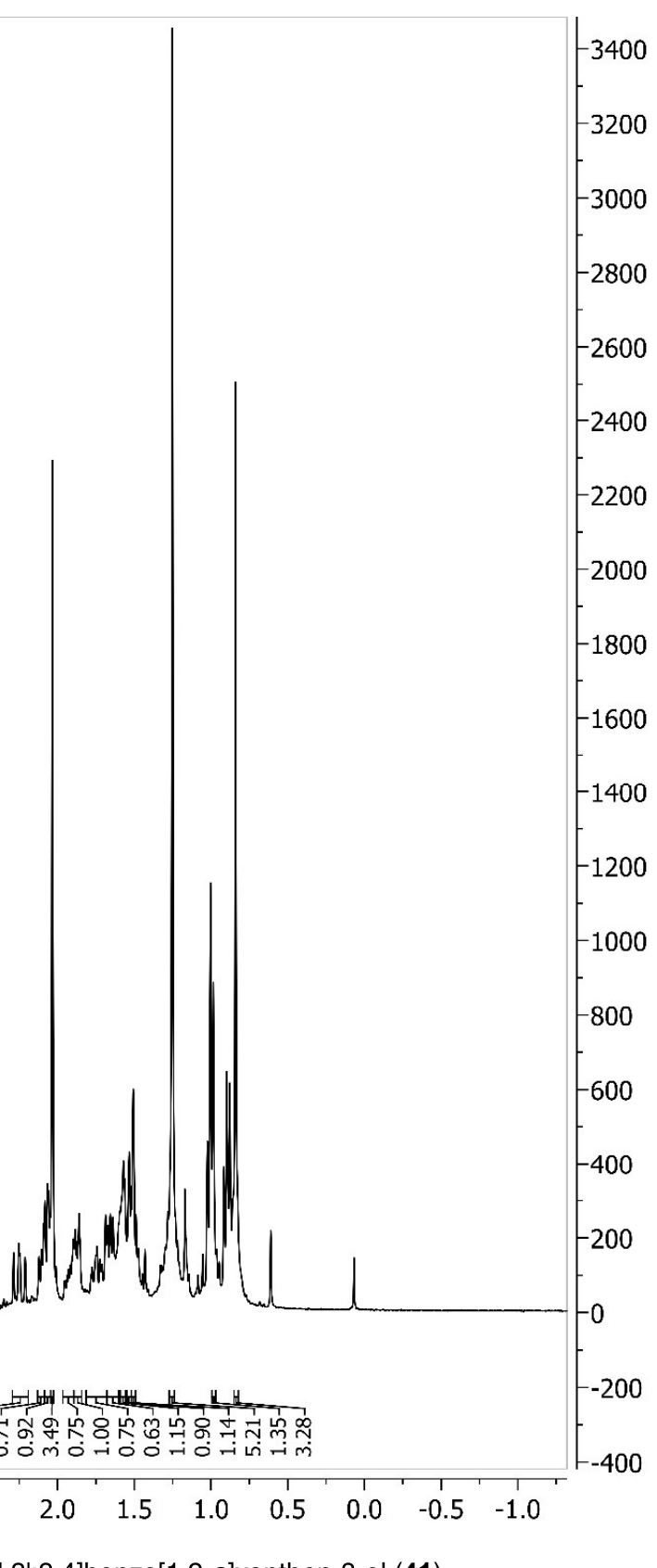




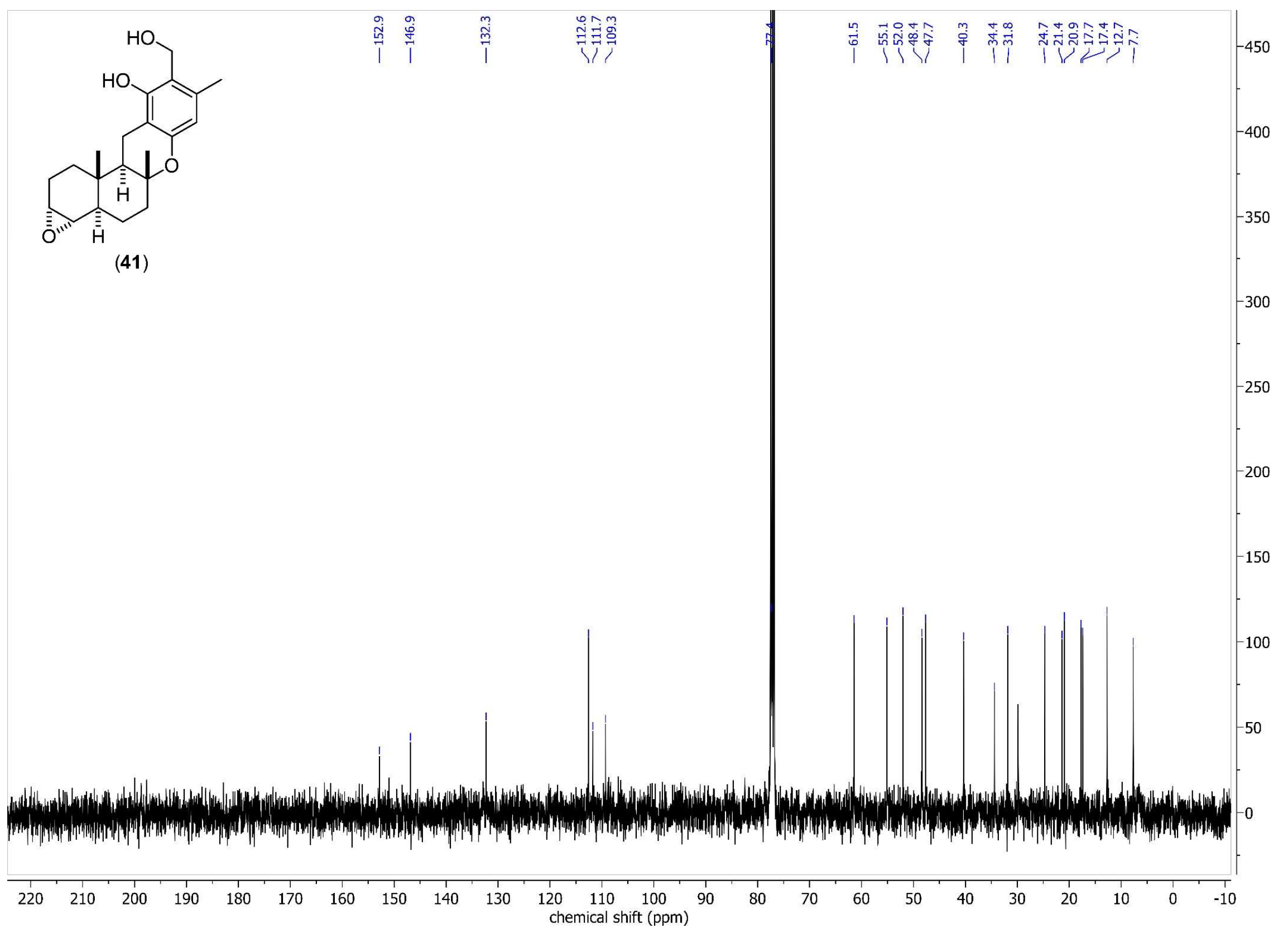

Figure S46: ${ }^{13} \mathrm{C}\{\mathrm{H}\}-\mathrm{NMR}\left(101 \mathrm{MHz}, \mathrm{CDCl}_{3}\right)$ spectrum of ( \pm )-7-(hydroxymethyl)-3a,6,9b-trimethyl-1a,1b,3,3a,9,9a,9b,10,11,11a-decahydro-2H-oxireno[2',3':3,4]benzo[1,2-a]xanthen-8-ol (41). S48 


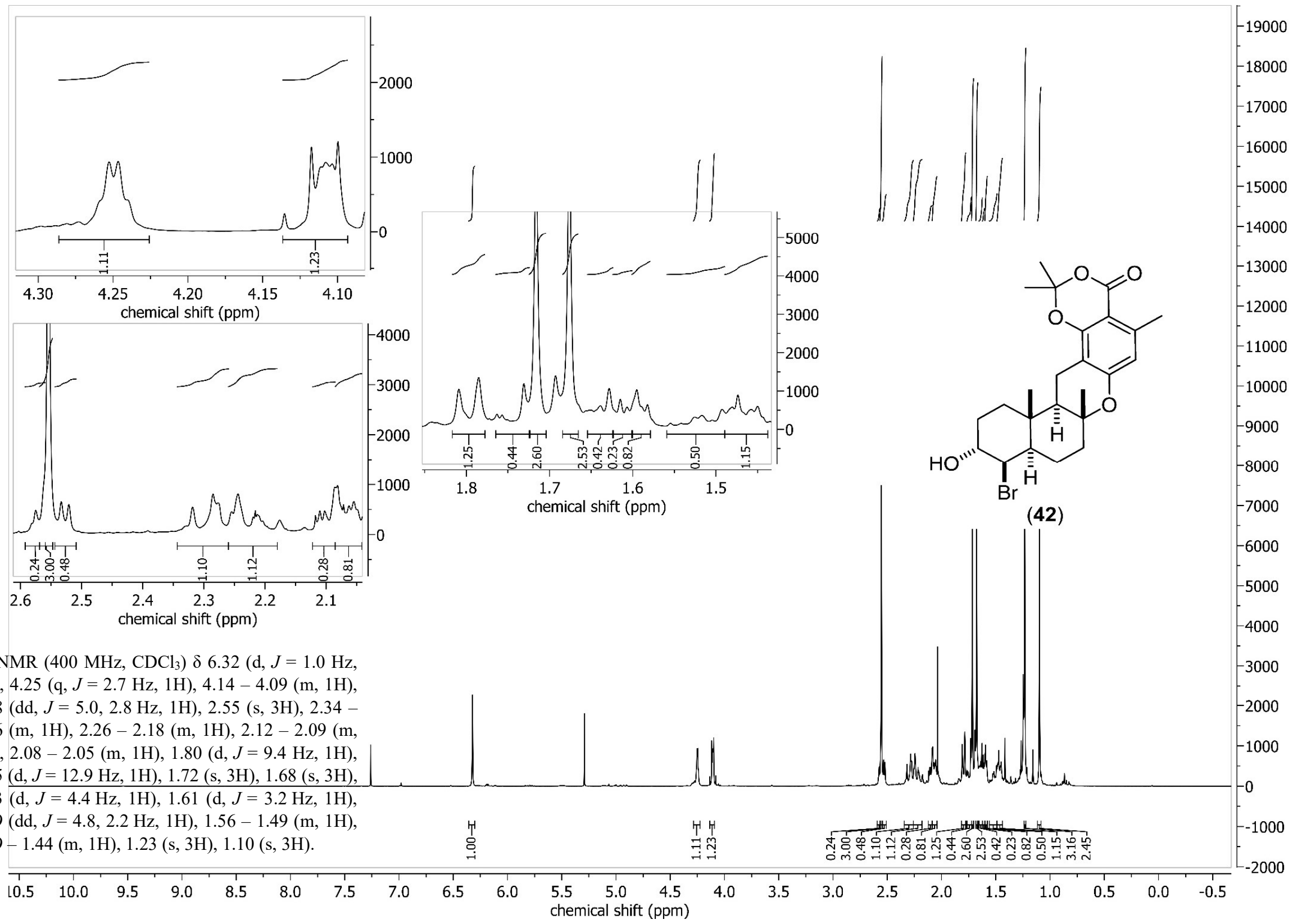

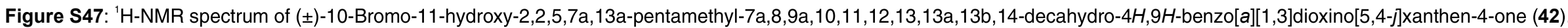




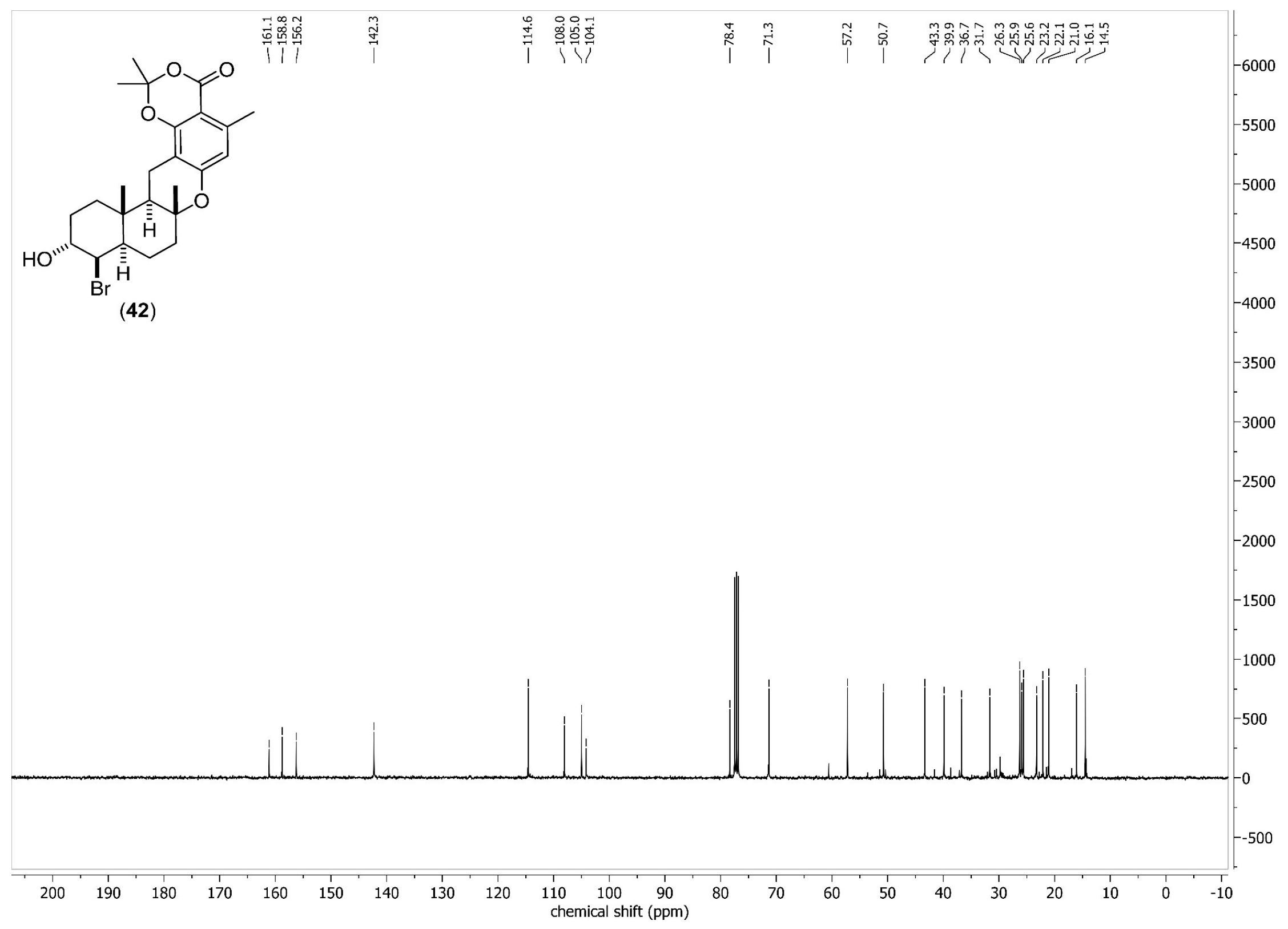

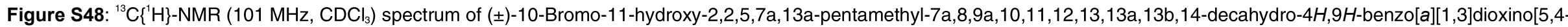
j]xanthen-4-one (42). 

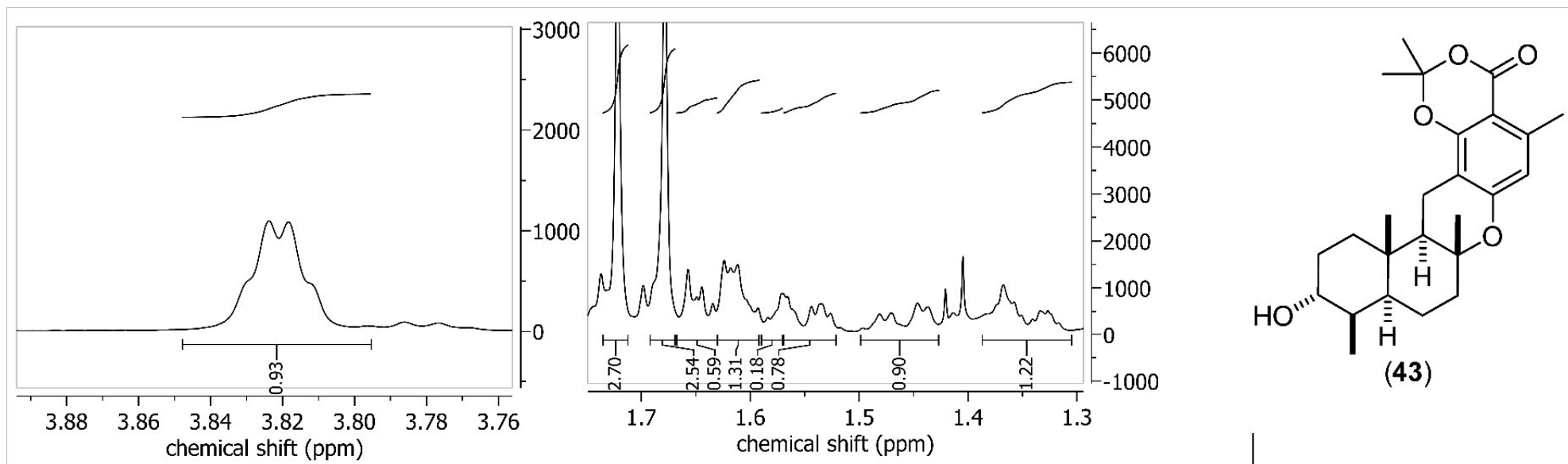

(43)

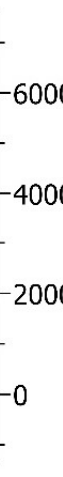

$\begin{array}{lllllllll}6 & 2.5 & 2.4 & 2.3 & 2.2 & 2.1 & 2.0 & 1.9 & 1.8\end{array}$ chemical shift (ppm)

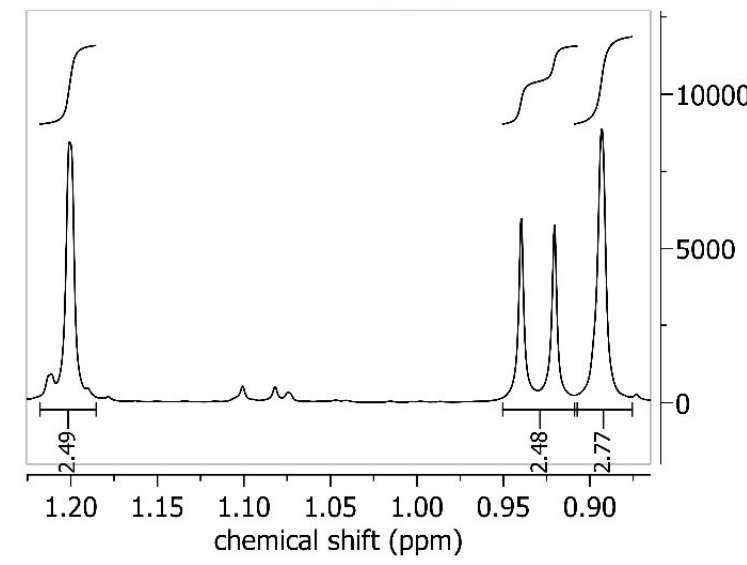

${ }^{1} \mathrm{H}-\mathrm{NMR}\left(400 \mathrm{MHz}, \mathrm{CDCl}_{3}\right) \delta 6.33(\mathrm{~d}, J=1.0 \mathrm{~Hz}, 1 \mathrm{H})$, $3.82(\mathrm{q}, J=2.6 \mathrm{~Hz}, 1 \mathrm{H}), 2.56(\mathrm{~s}, 3 \mathrm{H}), 2.52(\mathrm{~d}, J=5.0$ $\mathrm{Hz}, 1 \mathrm{H}), 2.28-2.19(\mathrm{~m}, 1 \mathrm{H}), 2.07(\mathrm{dt}, J=12.3,3.1 \mathrm{~Hz}$, 1H), $1.95-1.87(\mathrm{~m}, 1 \mathrm{H}), 1.85$ (ddd, $J=10.2,4.9,2.4$ $\mathrm{Hz}, 1 \mathrm{H}), 1.82-1.76(\mathrm{~m}, 1 \mathrm{H}), 1.72(\mathrm{~s}, 3 \mathrm{H}), 1.68(\mathrm{~s}, 3 \mathrm{H})$, $1.67-1.63(\mathrm{~m}, 1 \mathrm{H}), 1.63-1.59(\mathrm{~m}, 1 \mathrm{H}), 1.58-1.56$ $(\mathrm{m}, 1 \mathrm{H}), 1.59-1.52(\mathrm{~m}, 1 \mathrm{H}), 1.46(\mathrm{dd}, J=13.8,4.2 \mathrm{~Hz}$ $1 \mathrm{H}), 1.39-1.30(\mathrm{~m}, 1 \mathrm{H}), 1.20(\mathrm{~d}, J=0.9 \mathrm{~Hz}, 3 \mathrm{H}), 0.93$ $(\mathrm{d}, J=7.6 \mathrm{~Hz}, 3 \mathrm{H}), 0.89$ (s, 3H).

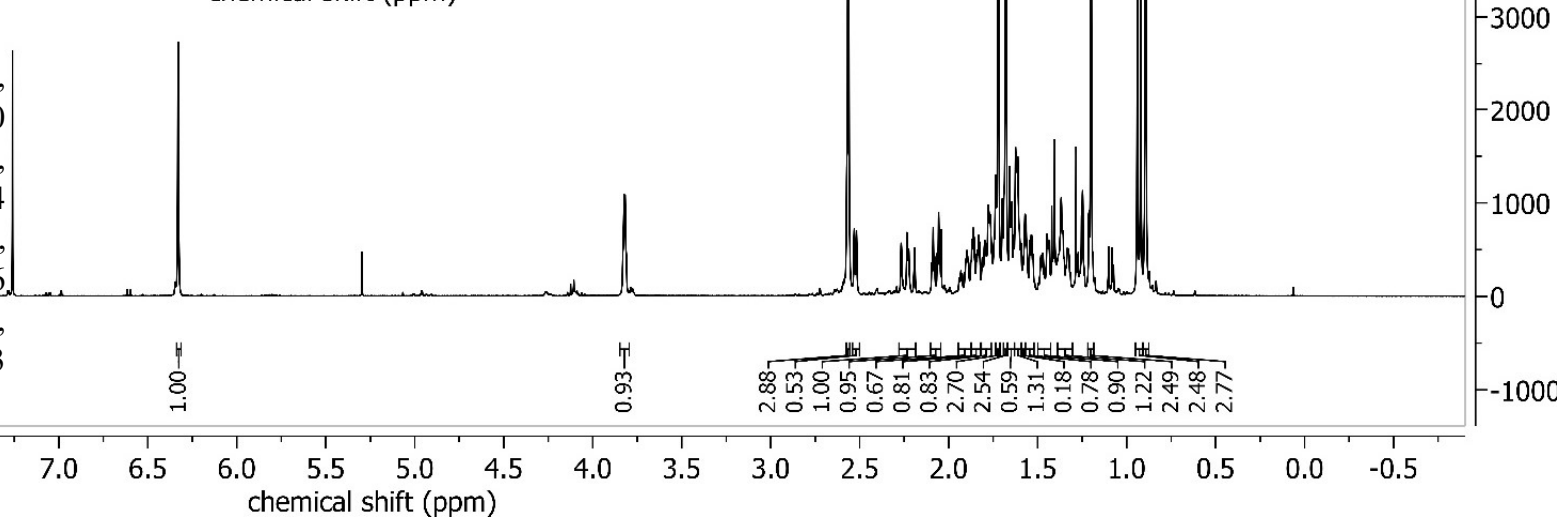

\footnotetext{
$\begin{array}{llllllll}11.0 & 10.5 & 10.0 & 9.5 & 9.0 & 8.5 & 8.0 & 7.5\end{array}$ chemical shift (ppm)
}

Figure S49: 'H-NMR spectrum of ( \pm )-11-Hydroxy-2,2,5,7a,10,13a-hexamethyl-7a,8,9a,10,11,12,13,13a,13b,14-decahydro-4H,9H-benzo[a][1,3]dioxino[5,4-j]xanthen-4-one (43). 


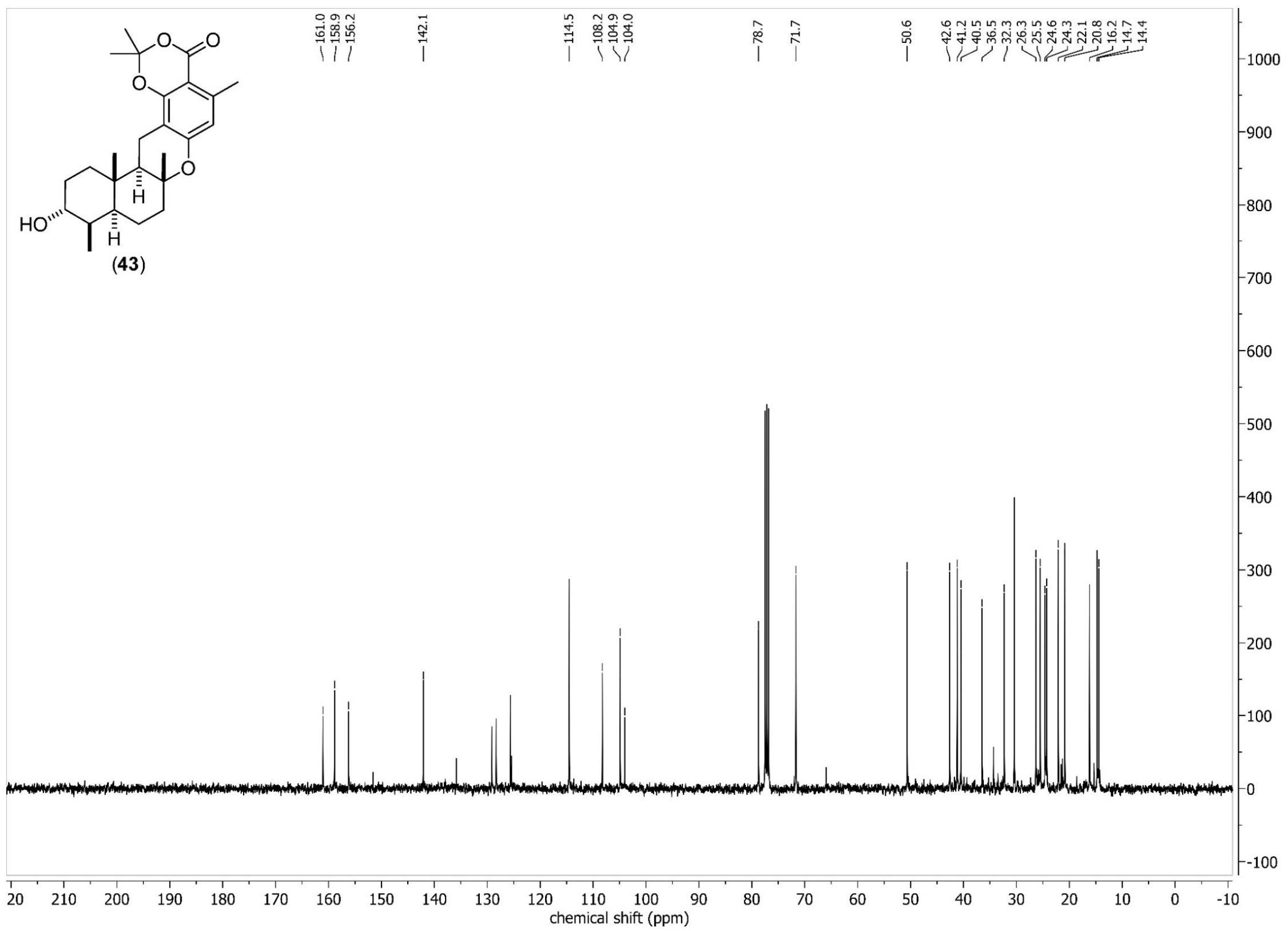

Figure S50: ${ }^{13} \mathrm{C}\left\{{ }^{1} \mathrm{H}\right\}-\mathrm{NMR}\left(101 \mathrm{MHz}, \mathrm{CDCl}_{3}\right)$ spectrum of $( \pm)$-11-Hydroxy-2,2,5,7a,10,13a-hexamethyl-7a,8,9a, 10,11,12,13,13a,13b,14-decahydro-4H,9H-benzo[a][1,3]dioxino[5,4-]xanthen-4-one (43). 


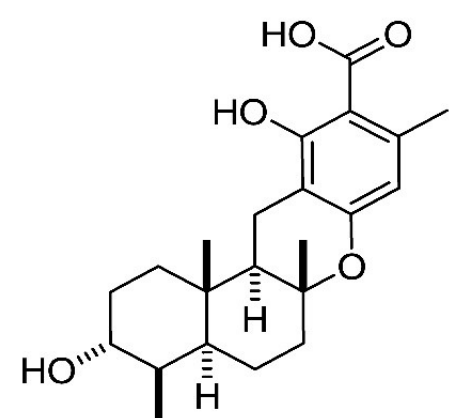

(44)
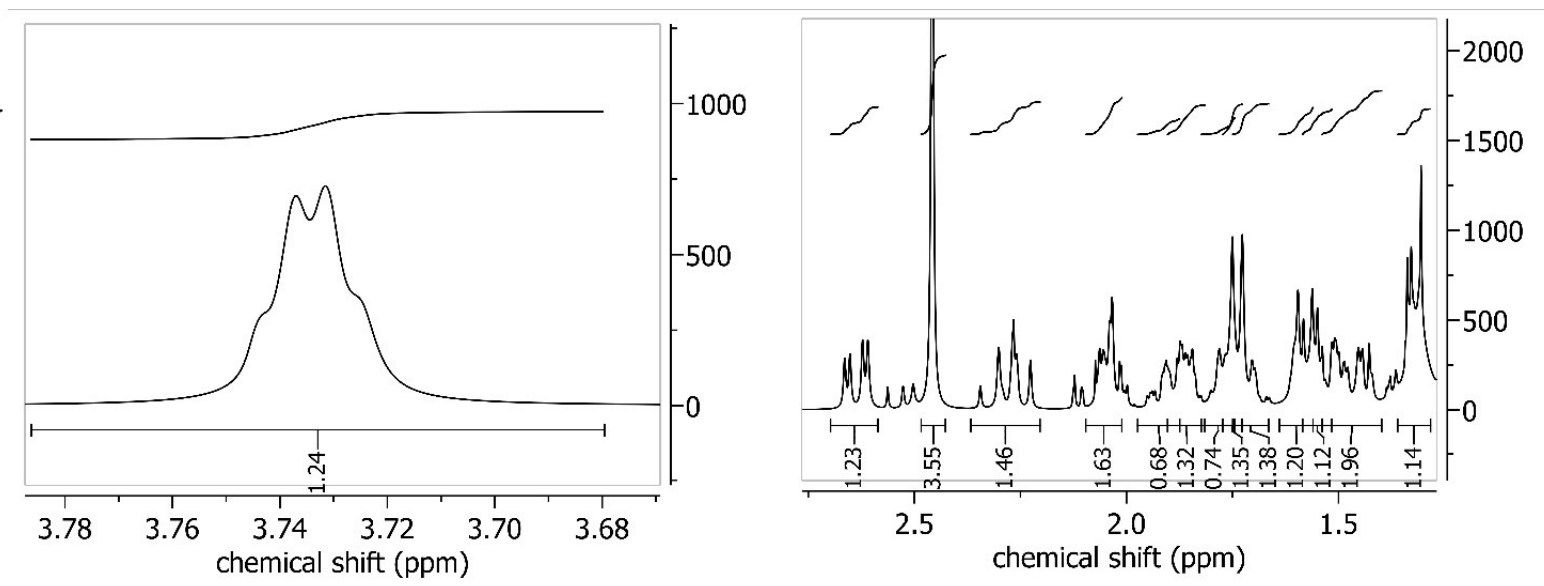

${ }^{1} \mathrm{H}-\mathrm{NMR}\left(400 \mathrm{MHz}, \mathrm{CD}_{3} \mathrm{OD}\right) \delta 6.11(\mathrm{~s}, 1 \mathrm{H}), 3.73(\mathrm{q}, J=2.7 \mathrm{~Hz}, 1 \mathrm{H}), 2.64$ $(\mathrm{dd}, J=16.9,5.0 \mathrm{~Hz}, 1 \mathrm{H}), 2.46(\mathrm{~s}, 3 \mathrm{H}), 2.35-2.21(\mathrm{~m}, 1 \mathrm{H}), 2.14-1.97(\mathrm{~m}$, $1 \mathrm{H}), 1.97-1.89(\mathrm{~m}, 1 \mathrm{H}), 1.86(\mathrm{ddd}, J=9.6,4.6,2.7 \mathrm{~Hz}, 1 \mathrm{H}), 1.81-1.76(\mathrm{~m}$, $1 \mathrm{H}), 1.76-1.73(\mathrm{~m}, 1 \mathrm{H}), 1.72(\mathrm{~d}, J=9.2 \mathrm{~Hz}, 1 \mathrm{H}), 1.59(\mathrm{t}, J=4.5 \mathrm{~Hz}, 1 \mathrm{H})$, $1.57-1.53(\mathrm{~m}, 1 \mathrm{H}), 1.53-1.41(\mathrm{~m}, 2 \mathrm{H}, 1.35-1.32(\mathrm{~m}, 1 \mathrm{H}), 1.19(\mathrm{~s}, 3 \mathrm{H})$, $0.95(\mathrm{~d}, J=7.9 \mathrm{~Hz}, 3 \mathrm{H}), 0.93(\mathrm{~s}, 3 \mathrm{H})$.

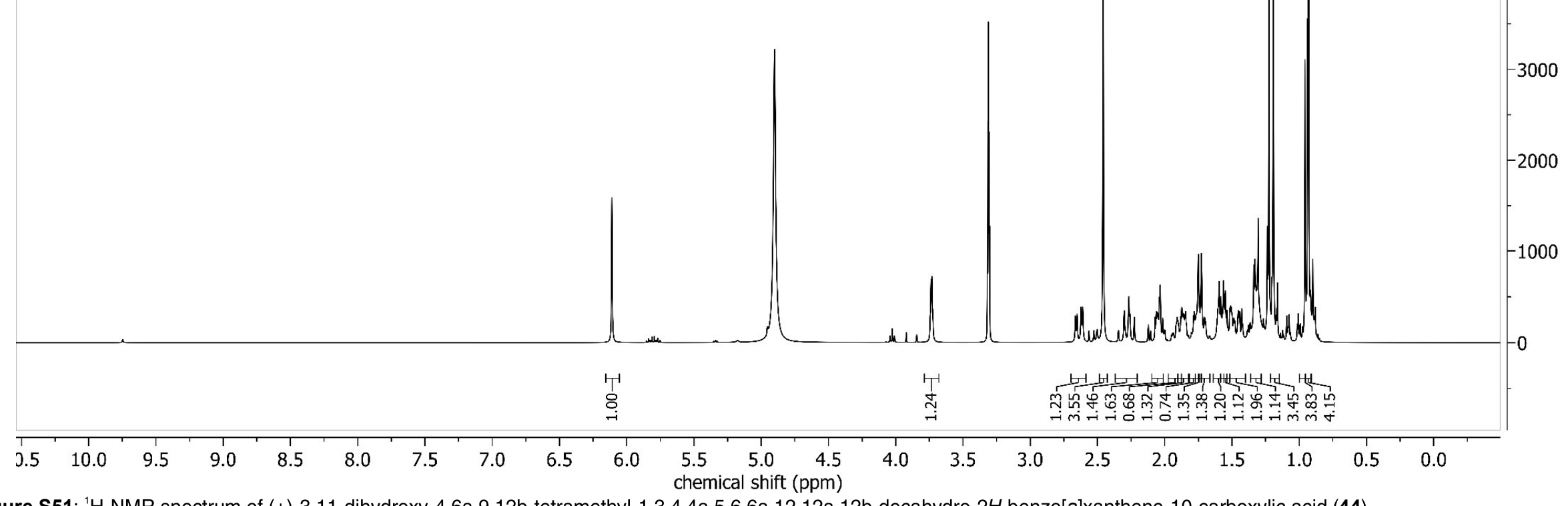

Figure S51: 'H-NMR spectrum of ( \pm )-3,11-dihydroxy-4,6a,9,12b-tetramethyl-1,3,4,4a,5,6,6a,12,12a,12b-decahydro-2H-benzo[a]xanthene-10-carboxylic acid (44). 


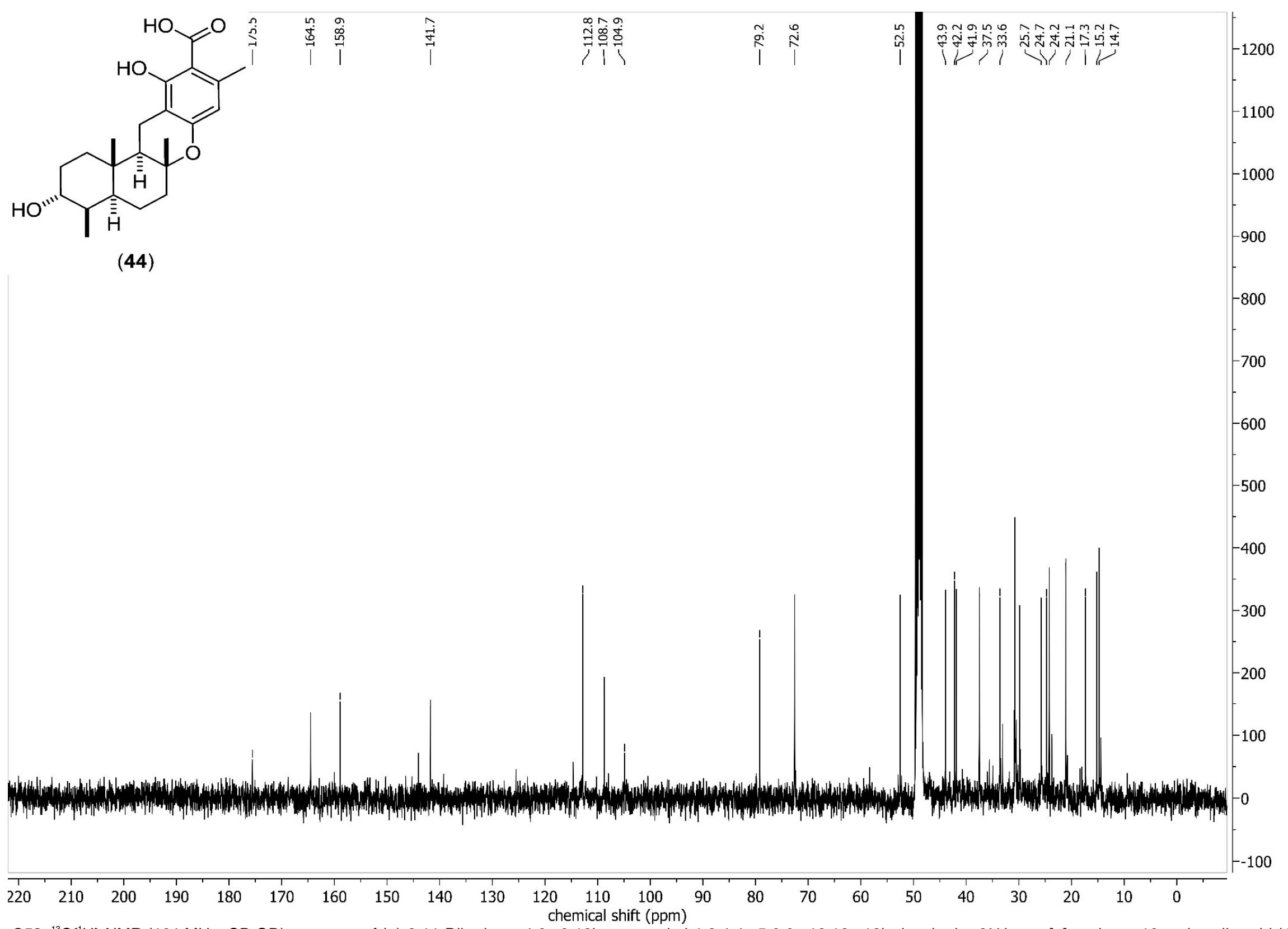

Figure S52: ${ }^{13} \mathrm{C}\{\mathrm{H}\}$-NMR $\left(101 \mathrm{MHz}, \mathrm{CD}_{3} \mathrm{OD}\right)$ spectrum of $( \pm)$-3,11-Dihydroxy-4,6a,9,12b-tetramethyl-1,3,4,4a,5,6,6a,12,12a, 12b-decahydro-2H-benzo[a]xanthene-10-carboxylic acid (44) 

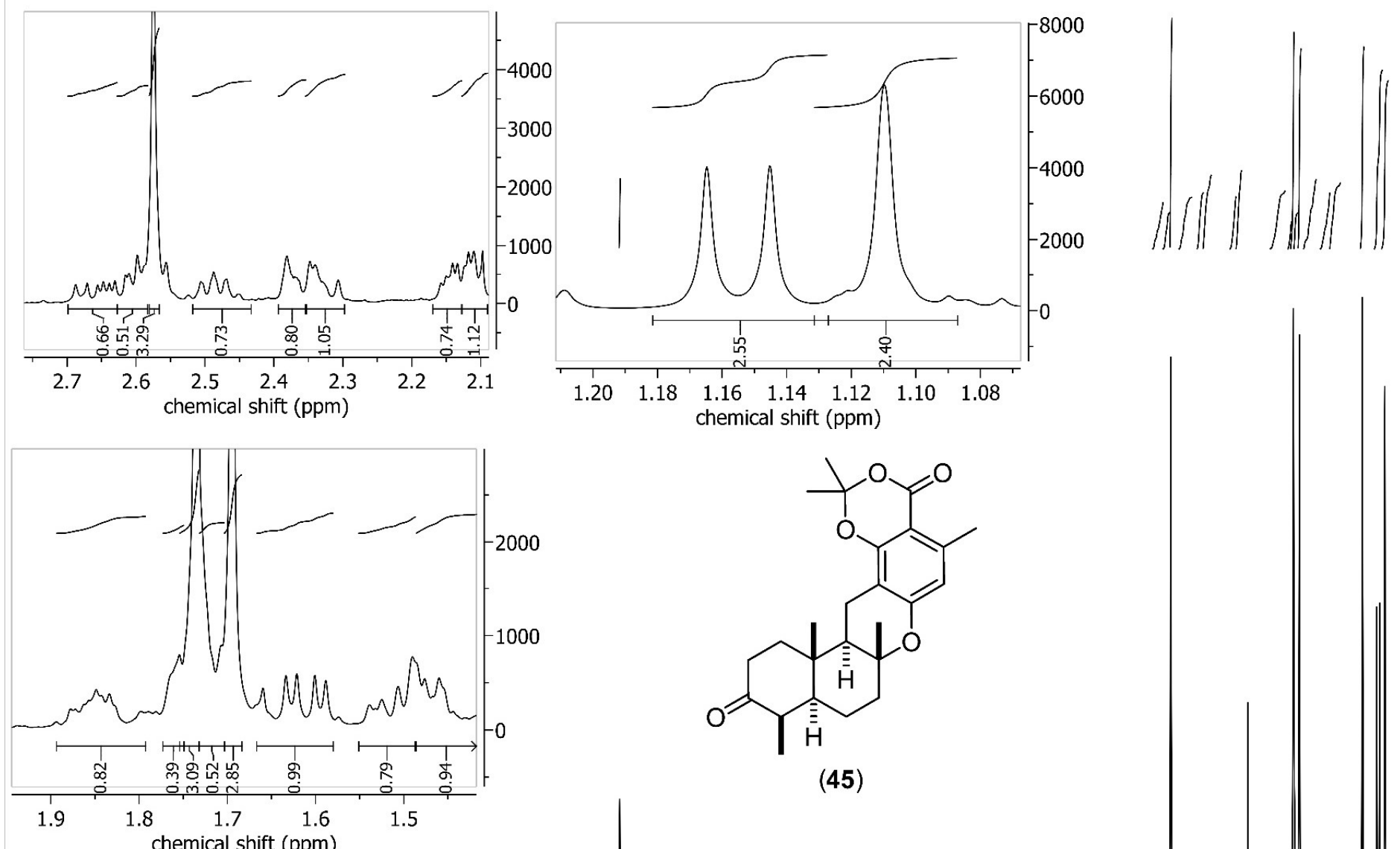

(45)

${ }^{1} \mathrm{H}-\mathrm{NMR}\left(400 \mathrm{MHz}, \mathrm{CDCl}_{3}\right) \delta 6.39-6.31(\mathrm{~m}, 1 \mathrm{H})$, $2.70-2.63(\mathrm{~m}, 1 \mathrm{H}) 263-2.58(\mathrm{~m}, 1 \mathrm{H}), 2.57(\mathrm{~s}, 3 \mathrm{H})$, $2.52-2.43(\mathrm{~m}, 1 \mathrm{H}), 2.39-2.35(\mathrm{~m}, 1 \mathrm{H}), 2.35-2.30$ (m, 1H), $2.15(\mathrm{dd}, J=6.6,3.2 \mathrm{~Hz}, 1 \mathrm{H}), 2.1(\mathrm{dd}, J=$ $7.0,3.7 \mathrm{~Hz}, 1 \mathrm{H}), 1.89-1.79(\mathrm{~m}, 1 \mathrm{H}), 1.77=1.75(\mathrm{~m}$,

1H), $1.74(\mathrm{~s}, 3 \mathrm{H}), 1.73(\mathrm{~d}, J=1.9 \mathrm{~Hz}, 1 \mathrm{H}), 1.69(\mathrm{~s}$, $3 \mathrm{H}), 1.67-1.58(\mathrm{~m}, 1 \mathrm{H}), 1.52(\mathrm{dd}, J=13.3,6.0 \mathrm{~Hz}$, 1H), $1.49-1.41(\mathrm{~m}, 1 \mathrm{H}), 1.26(\mathrm{~d}, J=1.4 \mathrm{~Hz}, 3 \mathrm{H})$, 1.15 (d. $J=7.8 \mathrm{~Hz} .3 \mathrm{H}), 1.11$ (s. $3 \mathrm{H})$.

$\begin{array}{lllllllllllll}0.5 & 10.0 & 9.5 & 9.0 & 8.5 & 8.0 & 7.5 & 7.0 & 6.5 & 6.0 & 5.5 & 5.0 & 4.5 \\ \text { chemical shift (ppm) }\end{array}$

Figure S53: 'H-NMR spectrum of $( \pm)-2,2,5,7 a, 10,13 a-H e x a m e t h y l-7 a, 8,9 a, 12,13,13 a, 13 b, 14-o c t a h y d r o-4 H, 9 H$-benzo[a][1,3]dioxino[5,4-]]xanthene-4,11(10H)-dione $(45)$ 


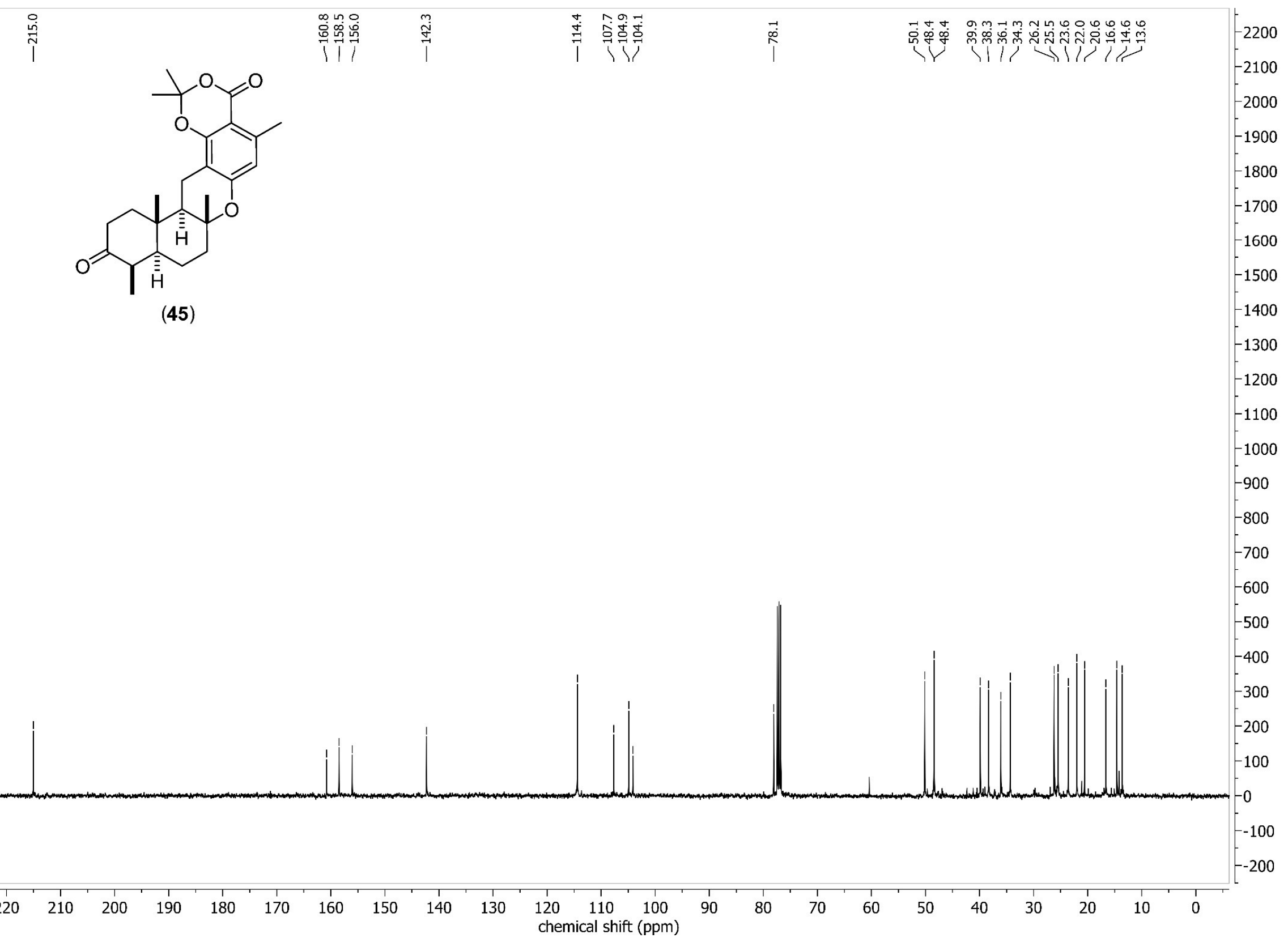

Figure S54: ${ }^{13} \mathrm{C}\left\{{ }^{1} \mathrm{H}\right\}-\mathrm{NMR}\left(101 \mathrm{MHz}, \mathrm{CDCl}_{3}\right)$ spectrum of $( \pm)-2,2,5,7 \mathrm{a}, 10,13 \mathrm{a}-$ Hexamethyl-7a,8,9a,12,13,13a, 13b,14-octahydro-4H,9H-benzo[a][1,3]dioxino[5,4-J]xanthene-4,11(10H)-dione (45). 


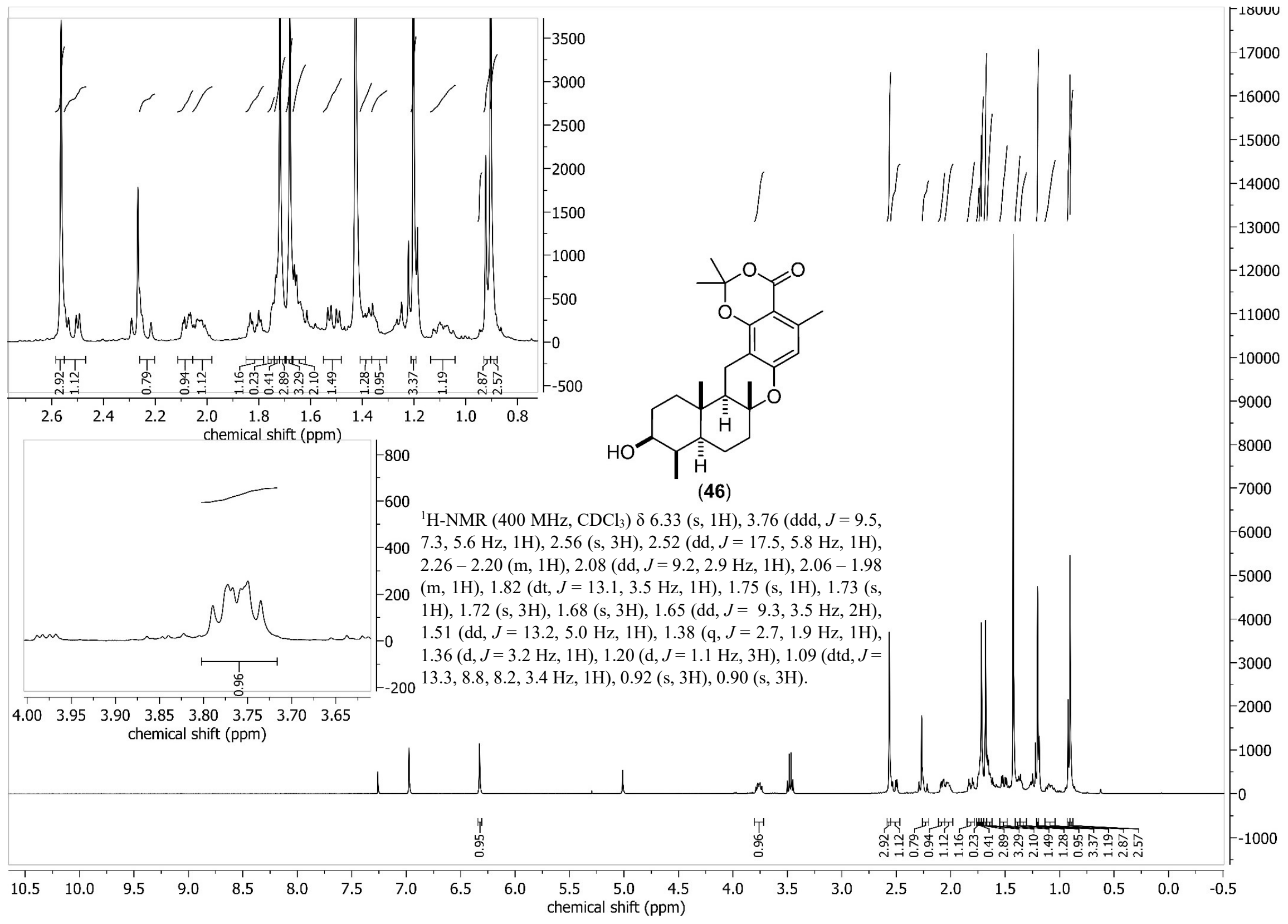

Figure S55: ${ }^{H} \mathrm{H}-\mathrm{NMR}$ spectrum of ( \pm )-11-Hydroxy-2,2,5,7a,10,13a-hexamethyl-7a,8,9a,10,11,12,13,13a,13b,14-decahydro-4H,9H-benzo[a][1,3]dioxino[5,4-j]xanthen-4-one (46). 


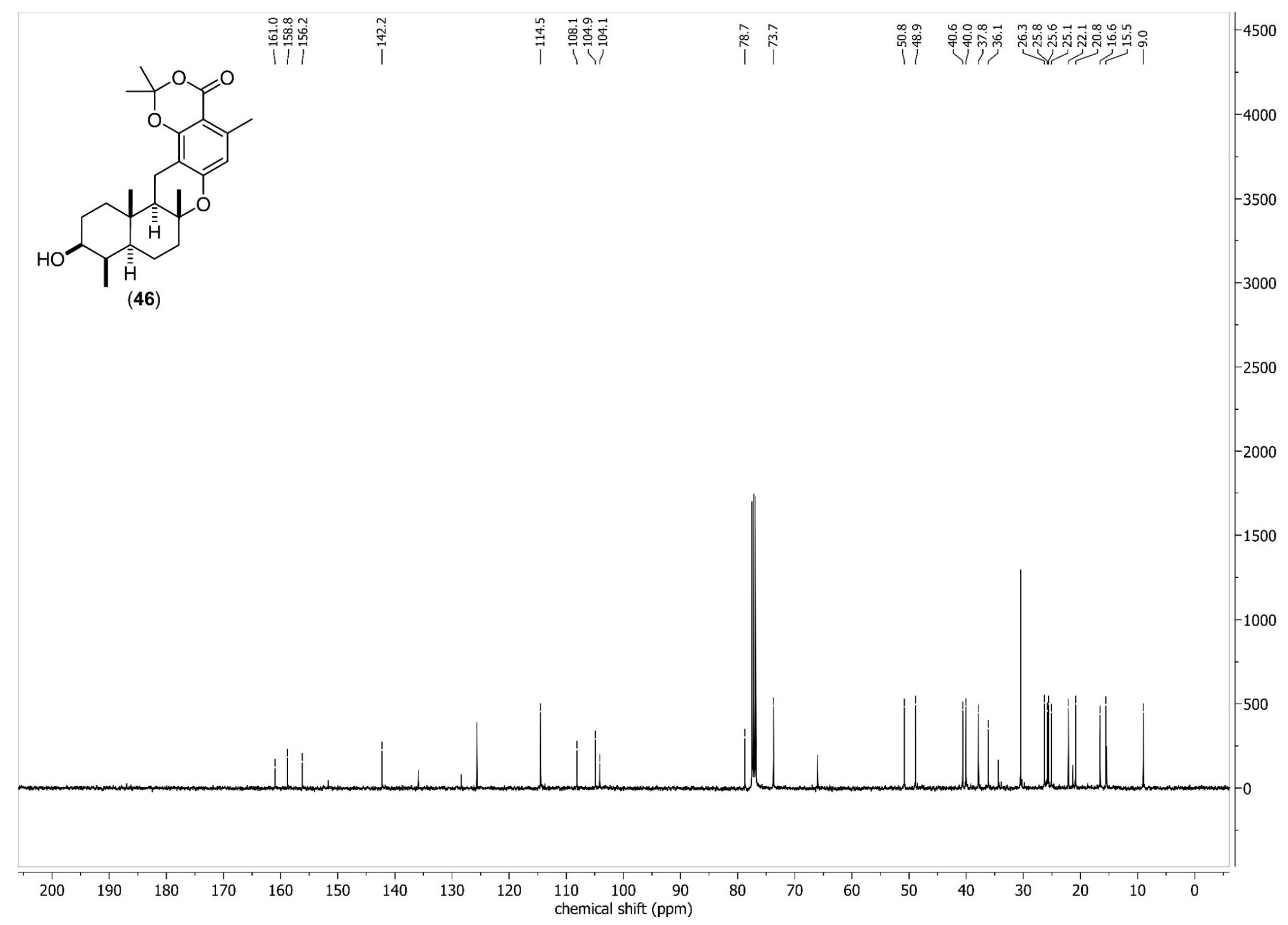

Figure S56: ${ }^{13} \mathrm{C}\left[{ }^{1} \mathrm{H}\right\}-\mathrm{NMR}\left(101 \mathrm{MHz}, \mathrm{CDCl}_{3}\right)$ spectrum of $( \pm)$-11-Hydroxy-2,2,5,7a,10,13a-hexamethyl-7a,8,9a, 10,11,12,13,13a, 13b, 14-decahydro-4H,9H-benzo[a][1,3]dioxino[5,4-j]xanthen-4-one (46). 


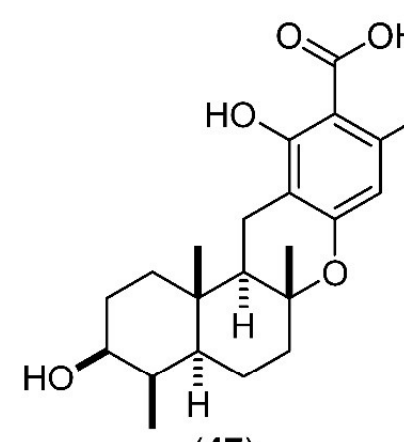

(47)

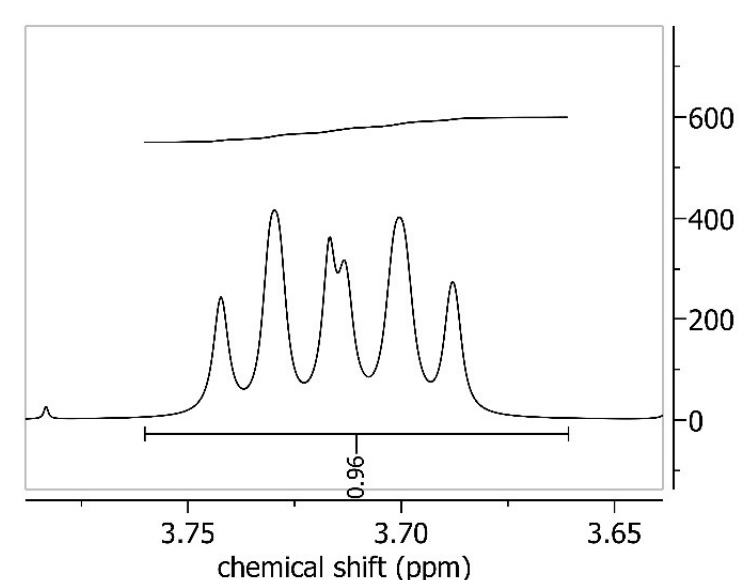

chemical shift (ppm)
${ }^{1} \mathrm{H}-\mathrm{NMR}\left(400 \mathrm{MHz}, \mathrm{CD}_{3} \mathrm{OD}\right) \delta 6.11(\mathrm{~d}, J=0.9$ zz, 1H), $3.72(\mathrm{dt}, J=11.6,5.0 \mathrm{~Hz}, 1 \mathrm{H}), 2.67-$ $2.57(\mathrm{~m}, 1 \mathrm{H}), 2.46(\mathrm{~s}, 3 \mathrm{H}), 2.35-2.24(\mathrm{~m}, 1 \mathrm{H})$, $2.08-2.02(\mathrm{~m}, 1 \mathrm{H}), 2.01-1.98(\mathrm{~m}, 1 \mathrm{H}), 1.81(\mathrm{dd}$ $J=8.6,4.9 \mathrm{~Hz}, 1 \mathrm{H}), 1.77(\mathrm{~d}, J=6.0 \mathrm{~Hz}, 1 \mathrm{H}),$, $-1.66(\mathrm{~m}, 1 \mathrm{H}), 1.62(\mathrm{tt}, J=9.2,4.2 \mathrm{~Hz}, 1 \mathrm{H}), 1.49$ $(\mathrm{dd}, J=13.1,5.0 \mathrm{~Hz}, 1 \mathrm{H}), 1.46-1.38(\mathrm{~m}, 3 \mathrm{H})$, $1.19(\mathrm{~d}, J=0.8 \mathrm{~Hz}, 3 \mathrm{H}), 1.18-1.07(\mathrm{~m}, 1 \mathrm{H}), 0.94$ $(\mathrm{d}, J=0.7 \mathrm{~Hz}, 3 \mathrm{H}), 0.91(\mathrm{~d}, J=7.5 \mathrm{~Hz}, 3 \mathrm{H})$.

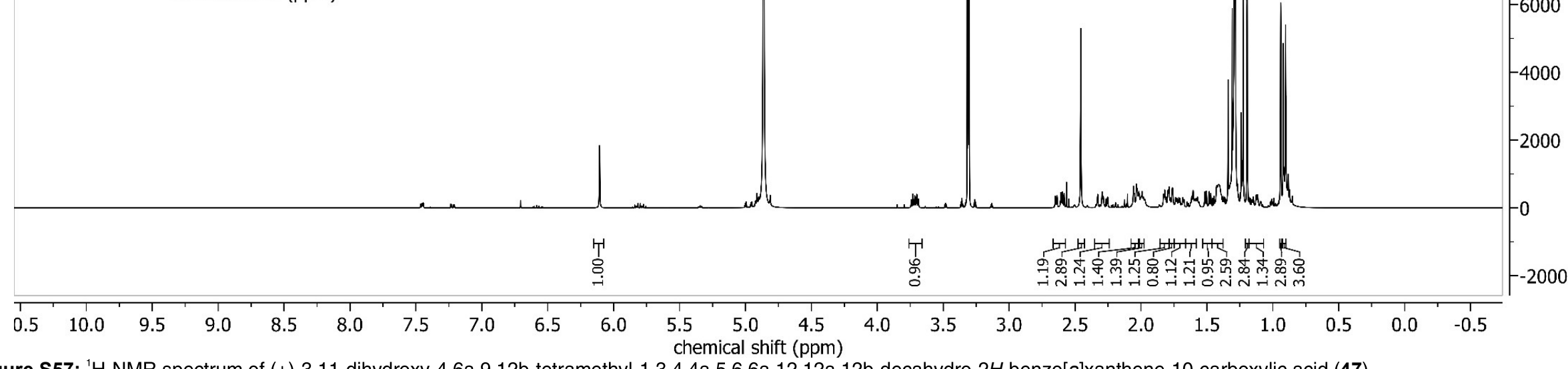

Figure S57: ${ }^{1} \mathrm{H}-\mathrm{NMR}$ spectrum of ( \pm )-3,11-dihydroxy-4,6a,9,12b-tetramethyl-1,3,4,4a,5,6,6a,12,12a,12b-decahydro-2H-benzo[a]xanthene-10-carboxylic acid (47).
28000

26000

24000

$-22000$

$-20000$

$-18000$

$-16000$

$-14000$

$-12000$

$-10000$

8000

6000

000

000 


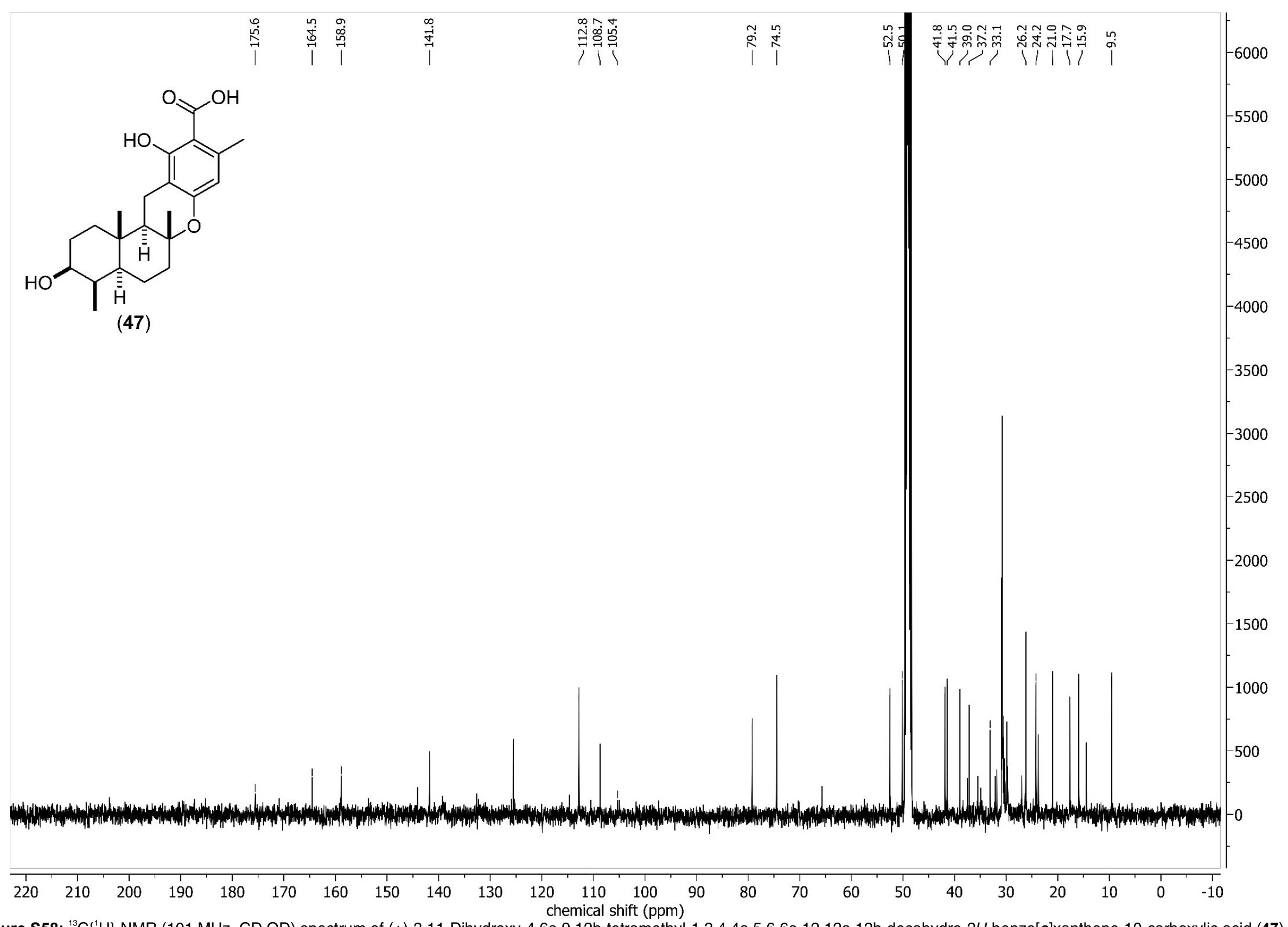

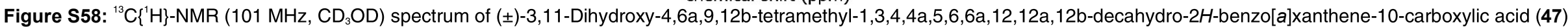




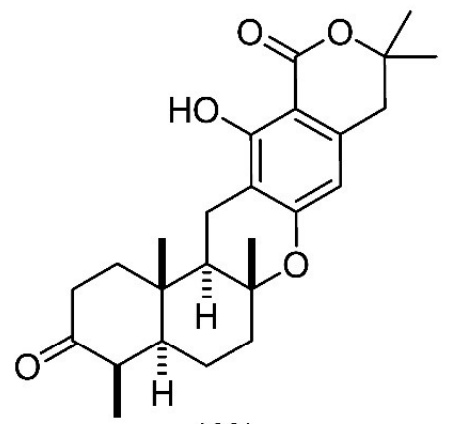

(48)

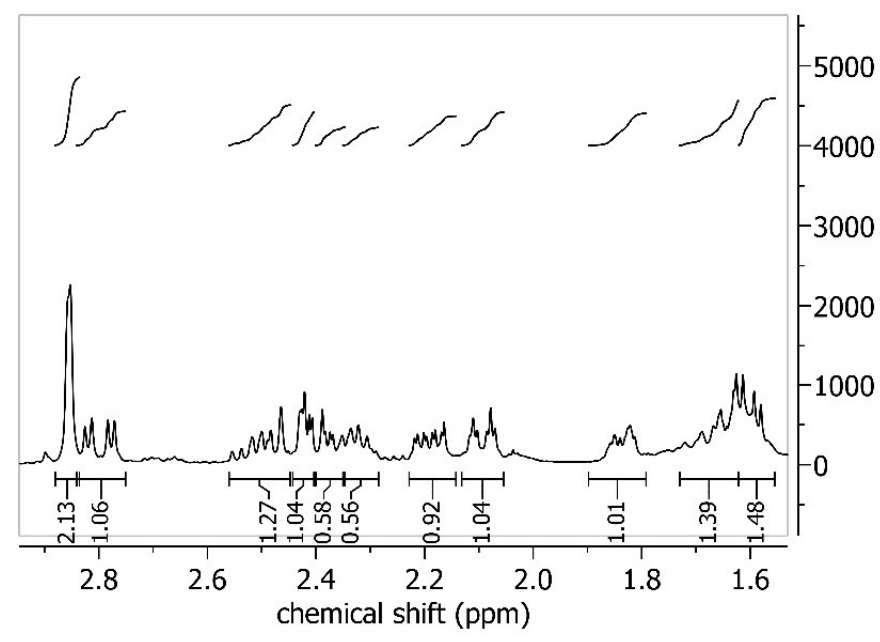

${ }^{1} \mathrm{H}-\mathrm{NMR}\left(400 \mathrm{MHz}, \mathrm{CDCl}_{3}\right) \delta 11.62(\mathrm{~s}, 1 \mathrm{H}), 6.12(\mathrm{~d}, J=1.0 \mathrm{~Hz}, 1 \mathrm{H}), 2.86(\mathrm{~d}, J=2.5 \mathrm{~Hz}$, $2 \mathrm{H}), 2.80(\mathrm{dd}, J=16.8,5.0 \mathrm{~Hz}, 1 \mathrm{H}), 2.56-2.45(\mathrm{~m}, 1 \mathrm{H}), 2.44-2.40(\mathrm{~m}, 1 \mathrm{H}), 2.40-2.35$ (m, 1H), $2.37-2.27(\mathrm{~m}, 1 \mathrm{H}), 2.23-2.14(\mathrm{~m}, 1 \mathrm{H}), 2.09(\mathrm{dq}, J=13.1,3.0 \mathrm{~Hz}, 1 \mathrm{H}), 1.84$ $(\mathrm{dq}, J=11.0,3.5,2.6 \mathrm{~Hz}, 1 \mathrm{H}), 1.73-1.62(\mathrm{~m}, 1 \mathrm{H}), 1.62-1.55(\mathrm{~m}, 1 \mathrm{H}), 1.46(\mathrm{~s}, 3 \mathrm{H}), 1.44$ (s, 3H), $1.40(\mathrm{dt}, J=7.2,3.6 \mathrm{~Hz}, 1 \mathrm{H}), 1.36(\mathrm{dq}, J=5.3,2.4 \mathrm{~Hz}, 1 \mathrm{H}), 1.33(\mathrm{~d}, J=2.1 \mathrm{~Hz}$, $1 \mathrm{H}), 1.29(\mathrm{~d}, J=0.9 \mathrm{~Hz}, 3 \mathrm{H}), 1.14(\mathrm{~s}, 3 \mathrm{H}), 1.04(\mathrm{~d}, J=6.6 \mathrm{~Hz}, 3 \mathrm{H})$.

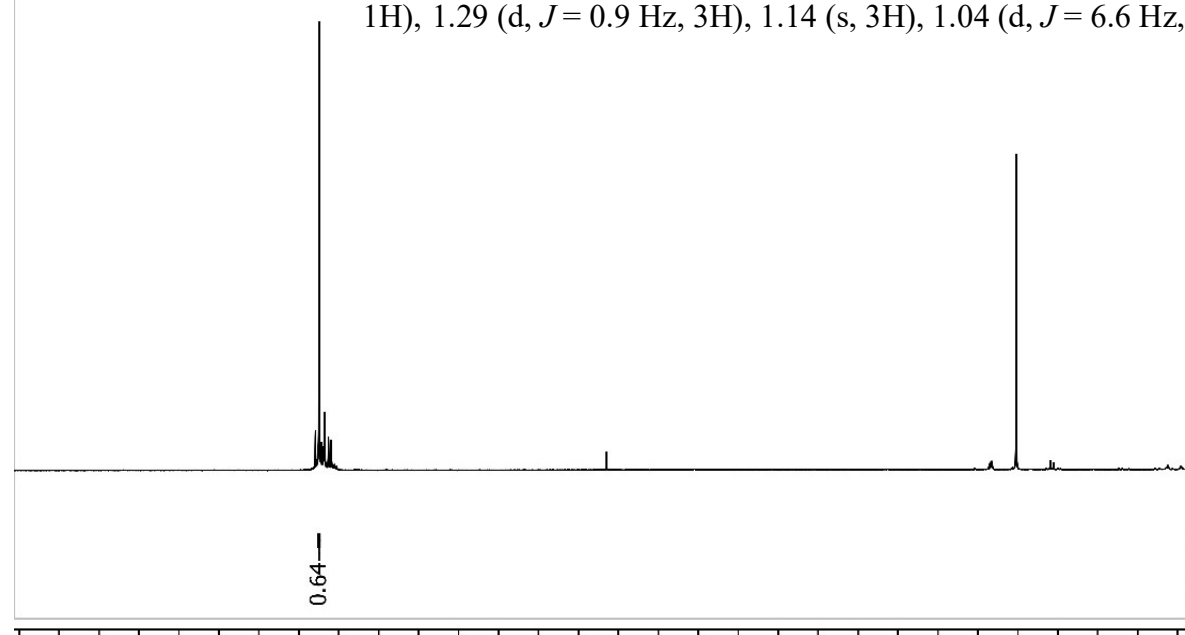

$-11000$ 10000 $-9000$ $-8000$ $-7000$ $-6000$ $-5000$ $-4000$ $-3000$ $-2000$ $-1000$

$\begin{array}{lllllllllllllllllllllllllllllll}3.5 & 13.0 & 12.5 & 12.0 & 11.5 & 11.0 & 10.5 & 10.0 & 9.5 & 9.0 & 8.5 & 8.0 & 7.5 & 7.0 & 6.5 & 6.0 & 5.5 & 5.0 & 4.5 & 4.0 & 3.5 & 3.0 & 2.5 & 2.0 & 1.5 & 1.0 & 0.5 & 0.0\end{array}$ chemical shift (ppm)

Figure S59: ${ }^{1} \mathrm{H}-\mathrm{NMR}$ spectrum of $( \pm)$-13-Hydroxy-4,6a,10,10,14b-pentamethyl-1,4,4a,5,6,6a,9,14,14a,14b-decahydro-2H,10H-benzo[a]pyrano[4,3-i]xanthene-3,12-dione $(48)$. 


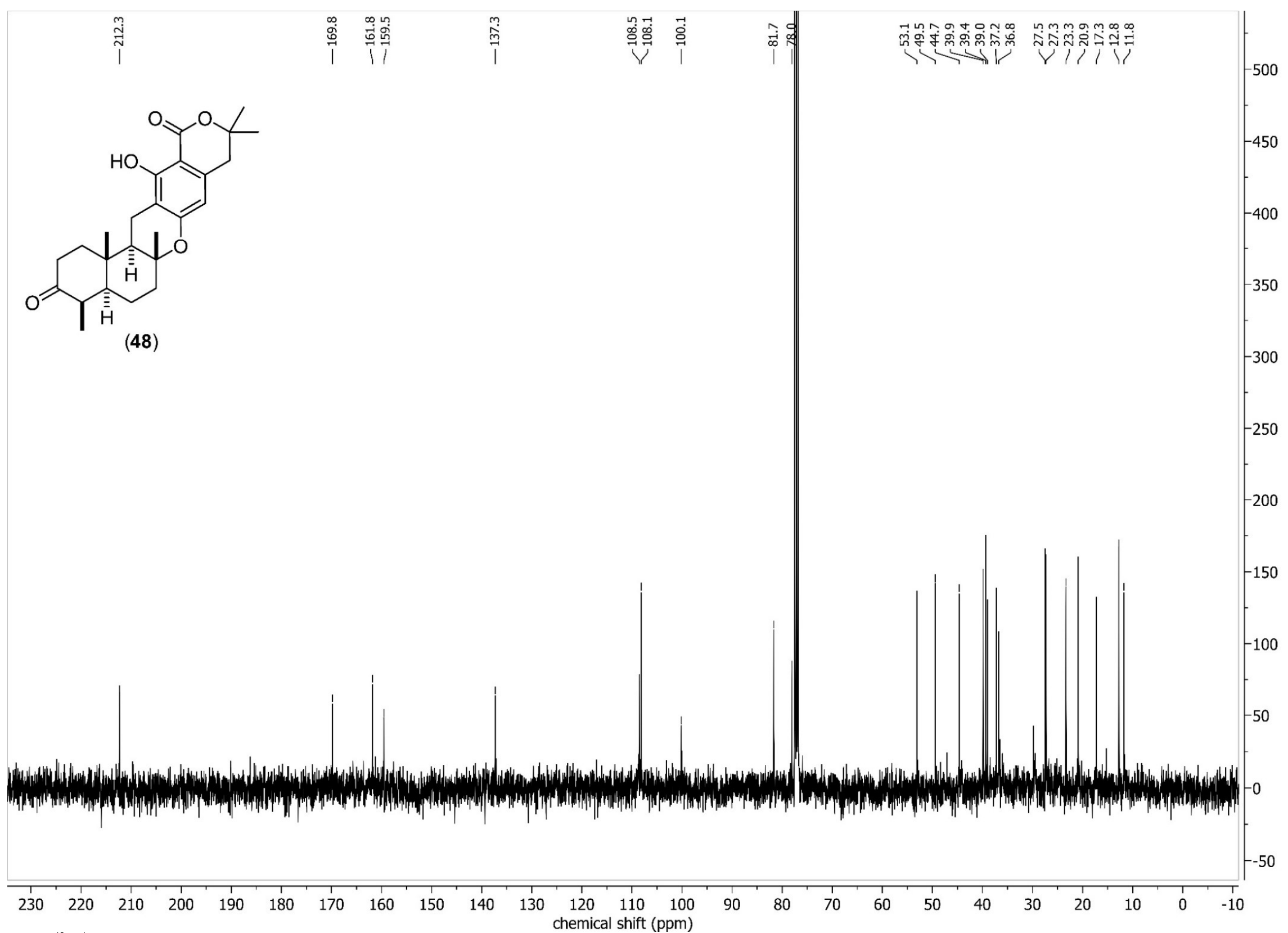

Figure S60: ${ }^{13} \mathrm{C}\left\{{ }^{1} \mathrm{H}\right\}-\mathrm{NMR}\left(101 \mathrm{MHz}, \mathrm{CDCl}_{3}\right)$ spectrum of $( \pm)-13-H y d r o x y-4,6 \mathrm{a}, 10,10,14 \mathrm{~b}$-pentamethyl-1,4,4a,5,6,6a,9,14,14a, 14b-decahydro-2H,10H-benzo[a]pyrano[4,3-1]xanthene-3,12-dione (48). 


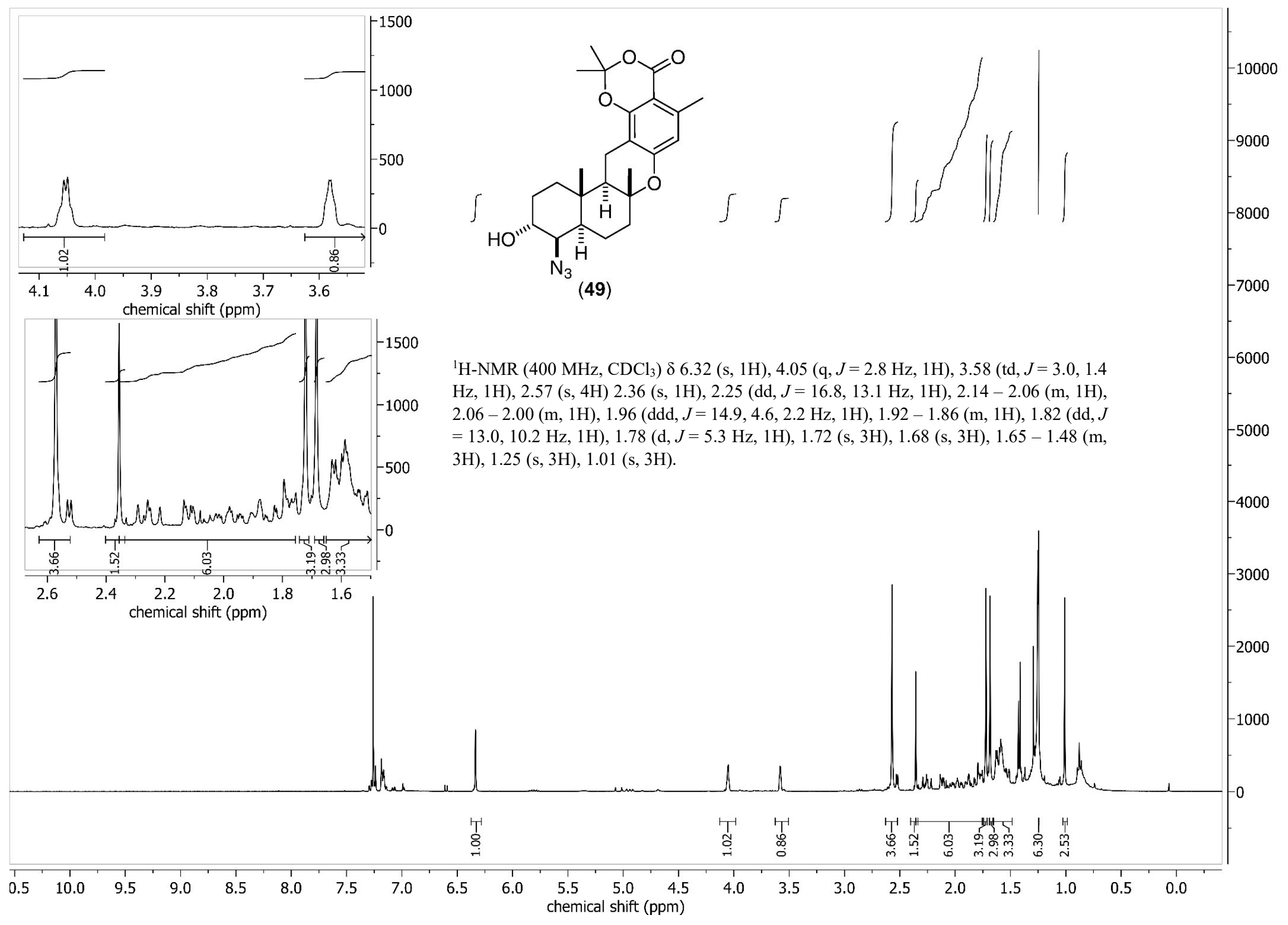

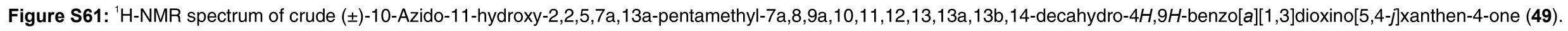




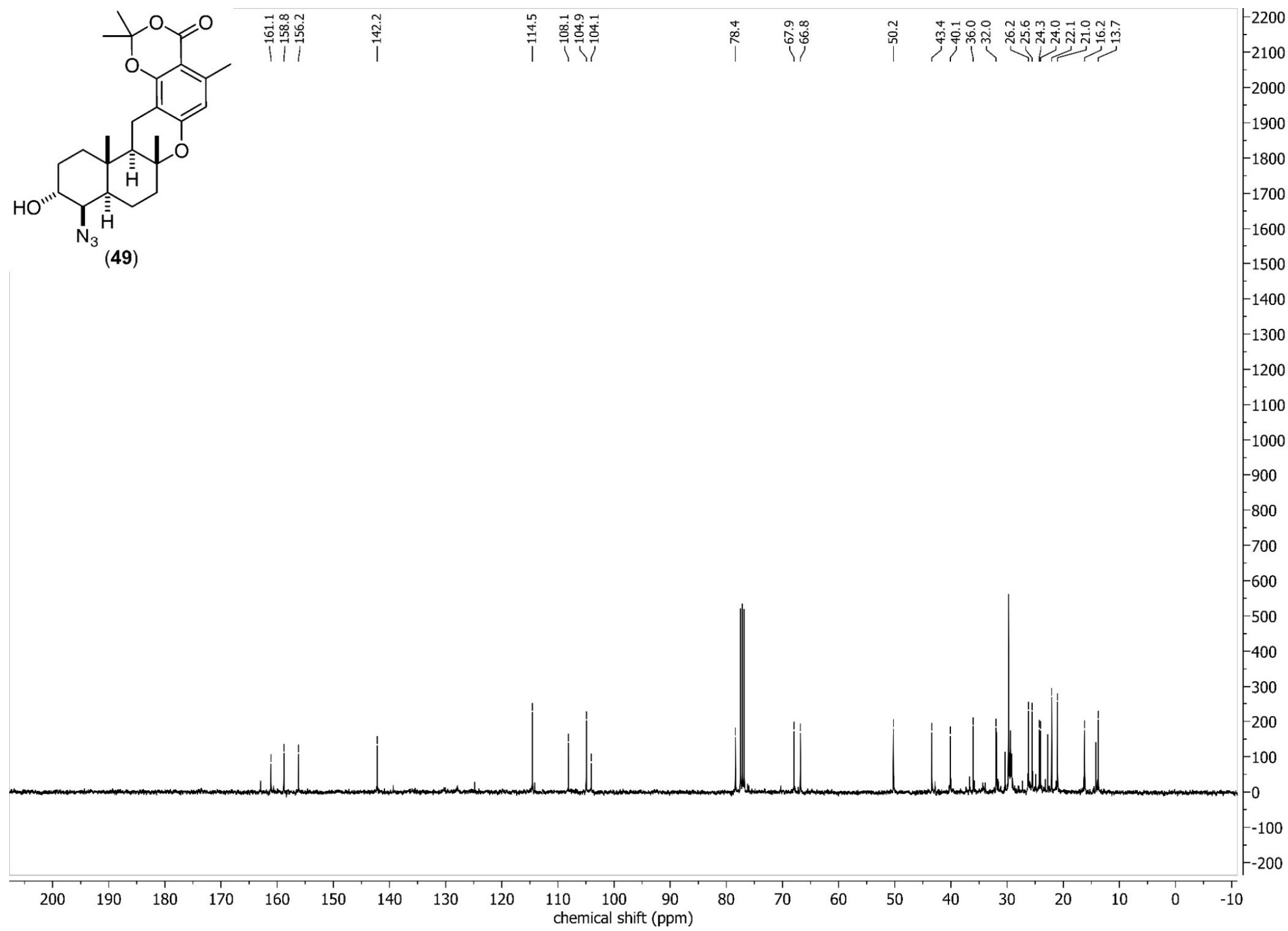

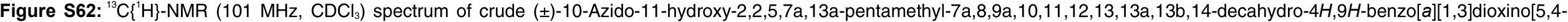
j]xanthen-4-one (49). 


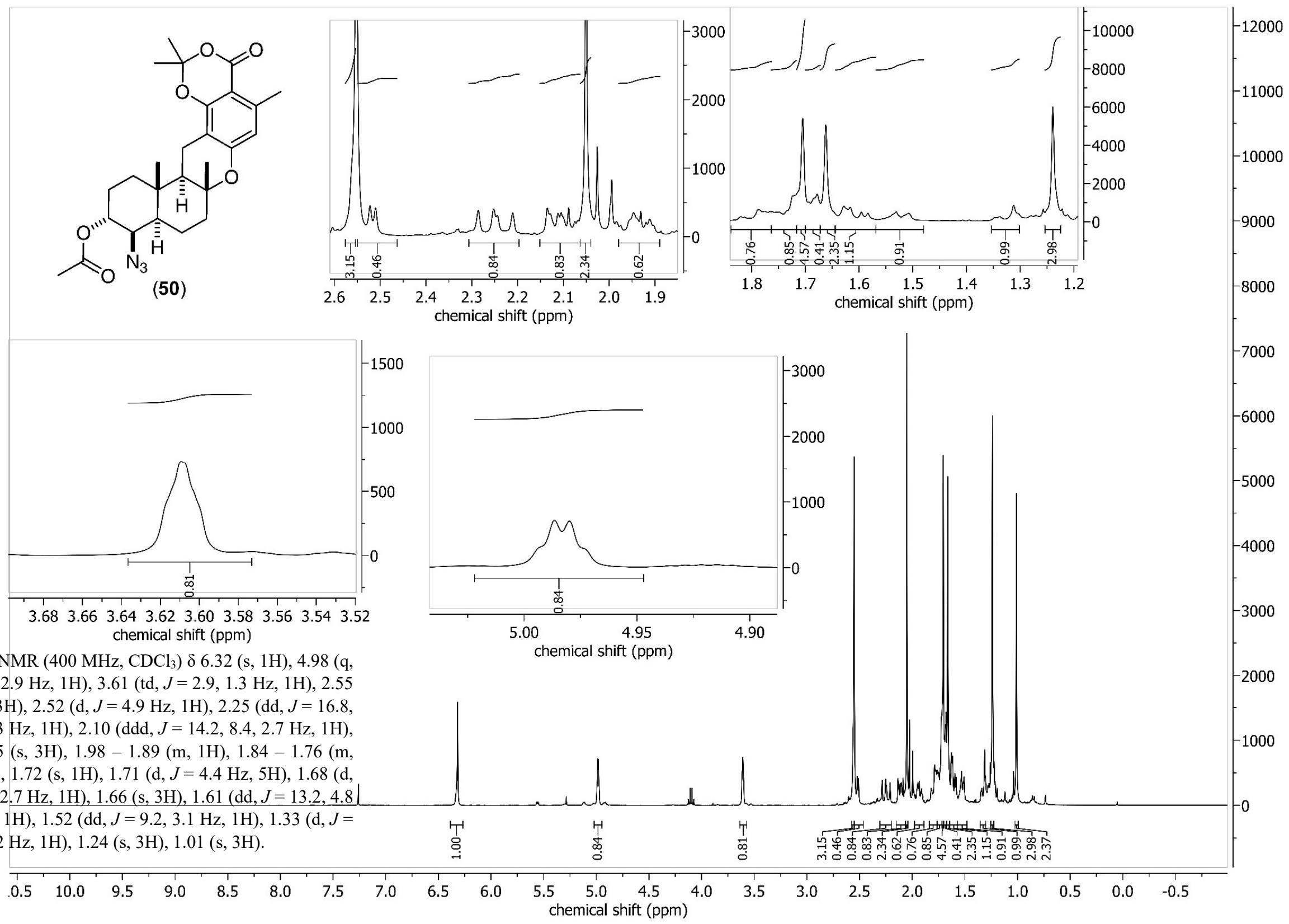

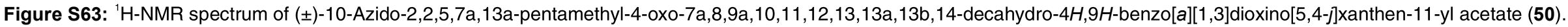




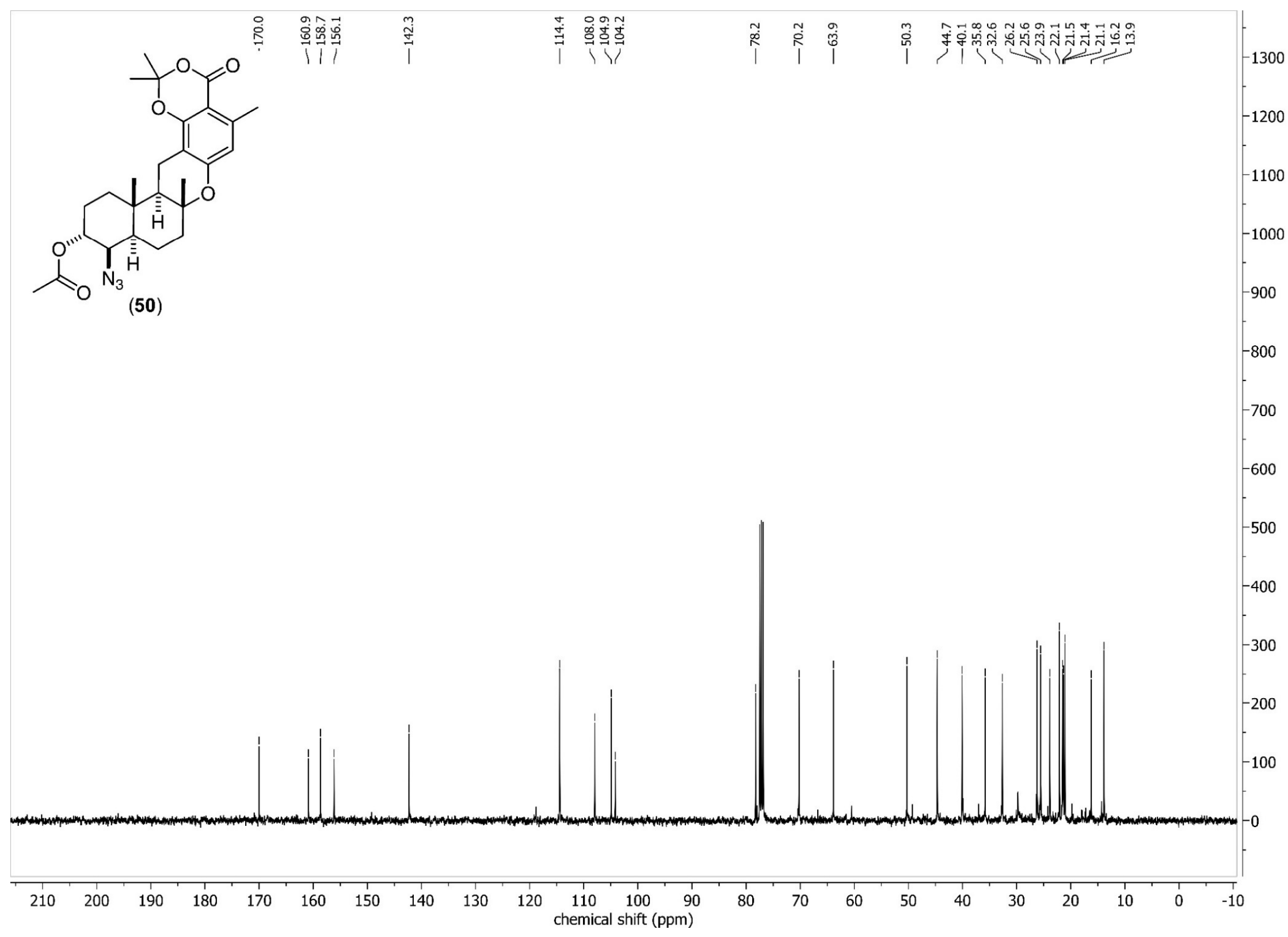

Figure S64: ${ }^{13} \mathrm{C}\left\{{ }^{1} \mathrm{H}\right\}-\mathrm{NMR}\left(101 \mathrm{MHz}, \mathrm{CDCl}_{3}\right)$ spectrum of ( \pm )-10-Azido-2,2,5,7a, 13a-pentamethyl-4-oxo-7a,8,9a, 10,11,12,13,13a,13b, 14-decahydro-4H,9H-benzo[a][1,3]dioxino[5,4-J]xanthen-11-yl acetate (50). 


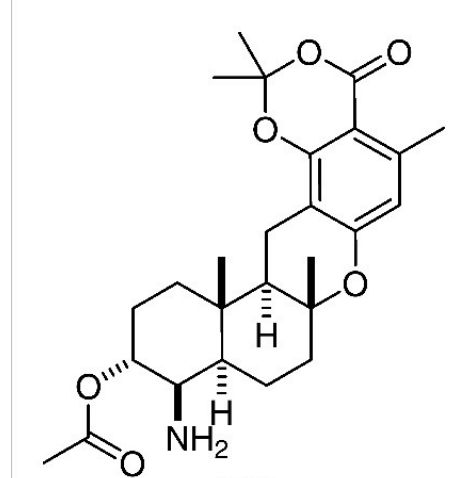

(51)
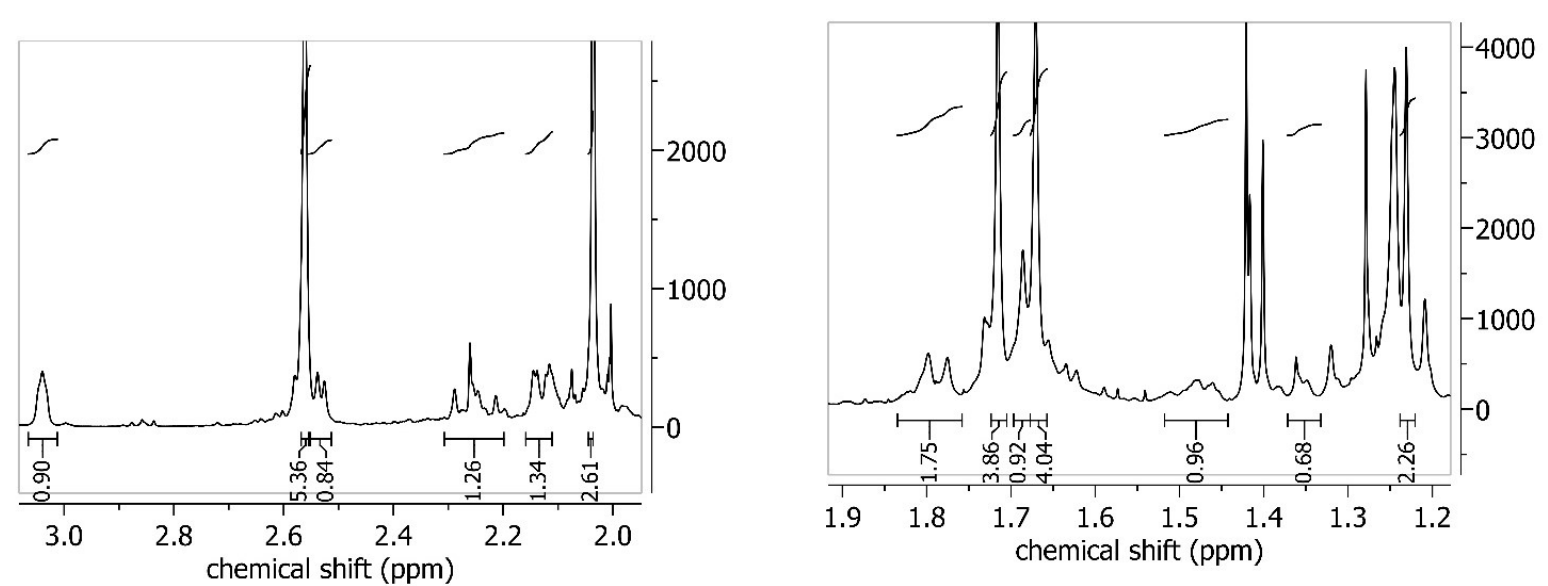

$-15000$

14000

$-13000$

12000

$-11000$

10000

9000

${ }^{1} \mathrm{H}-\mathrm{NMR}\left(400 \mathrm{MHz}, \mathrm{CDCl}_{3}\right) \delta 6.33(\mathrm{~d}, J=0.9 \mathrm{~Hz}, 1 \mathrm{H}), 5.08-4.90$

$\left(\mathrm{m}, 5 \mathrm{H}, \mathrm{NH}_{2}\right), 4.84(\mathrm{q}, J=2.7 \mathrm{~Hz}, 1 \mathrm{H}, \mathrm{H}), 3.04(\mathrm{~d}, J=3.3 \mathrm{~Hz}, 1 \mathrm{H})$,

$2.56(\mathrm{~s}, 5 \mathrm{H}), 2.53(\mathrm{~d}, J=5.1 \mathrm{~Hz}, 1 \mathrm{H}), 2.31-2.20(\mathrm{~m}, 1 \mathrm{H}), 2.16-$

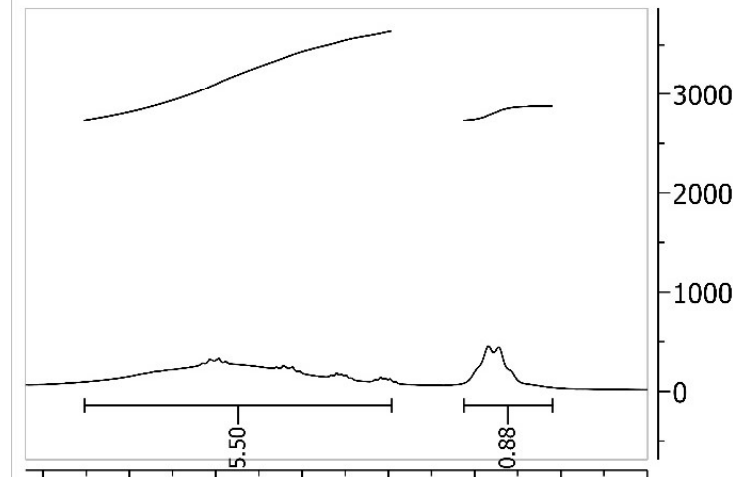

$2.06(\mathrm{~m}, 1 \mathrm{H}), 2.04(\mathrm{~s}, 3 \mathrm{H}), 1.83-1.76(\mathrm{~m}, 2 \mathrm{H}), 1.72(\mathrm{~s}, 4 \mathrm{H}), 1.69$

(s, $1 \mathrm{H}), 1.67$ (s, 4H), 1.48 (ddd, $J=9.3,7.1,4.6 \mathrm{~Hz}, 1 \mathrm{H}), 1.37-1.33$

$(\mathrm{m}, 1 \mathrm{H}), 1.23(\mathrm{~s}, 3 \mathrm{H}), 1.07(\mathrm{~s}, 3 \mathrm{H})$.

$\begin{array}{llllllll}5.10 & 5.05 & 5.00 & 4.95 & 4.90 & 4.85 & 4.80 & 4.75\end{array}$

chemical shift (ppm)

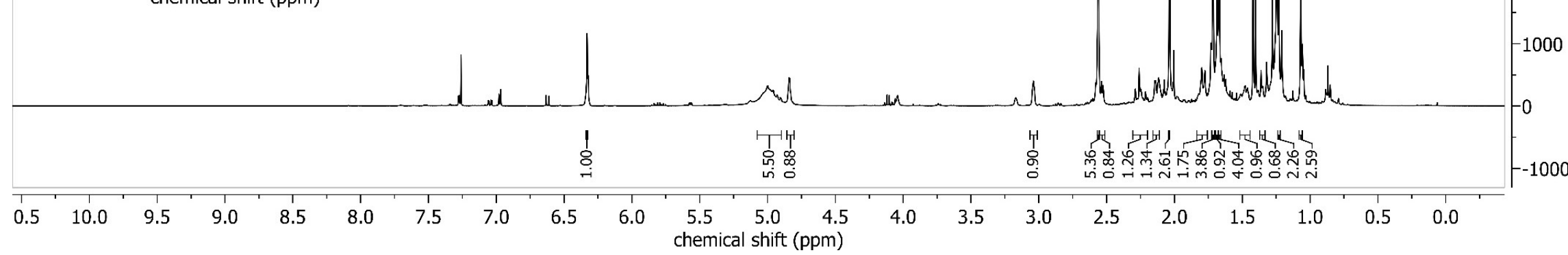

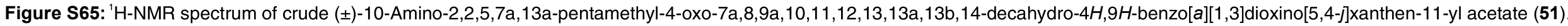




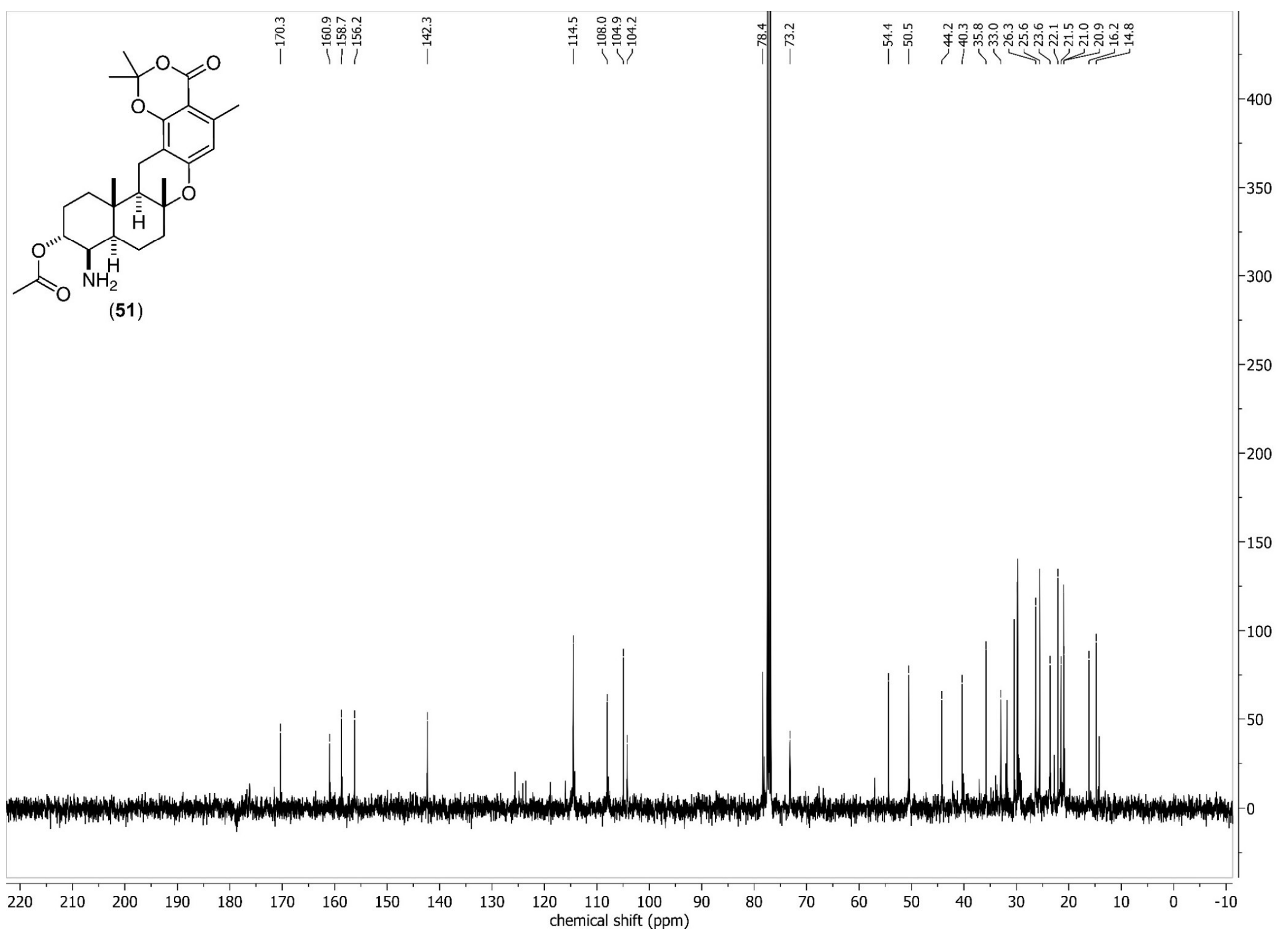

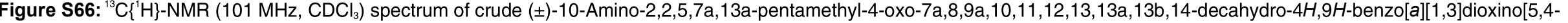
j]xanthen-11-yl acetate (51) 


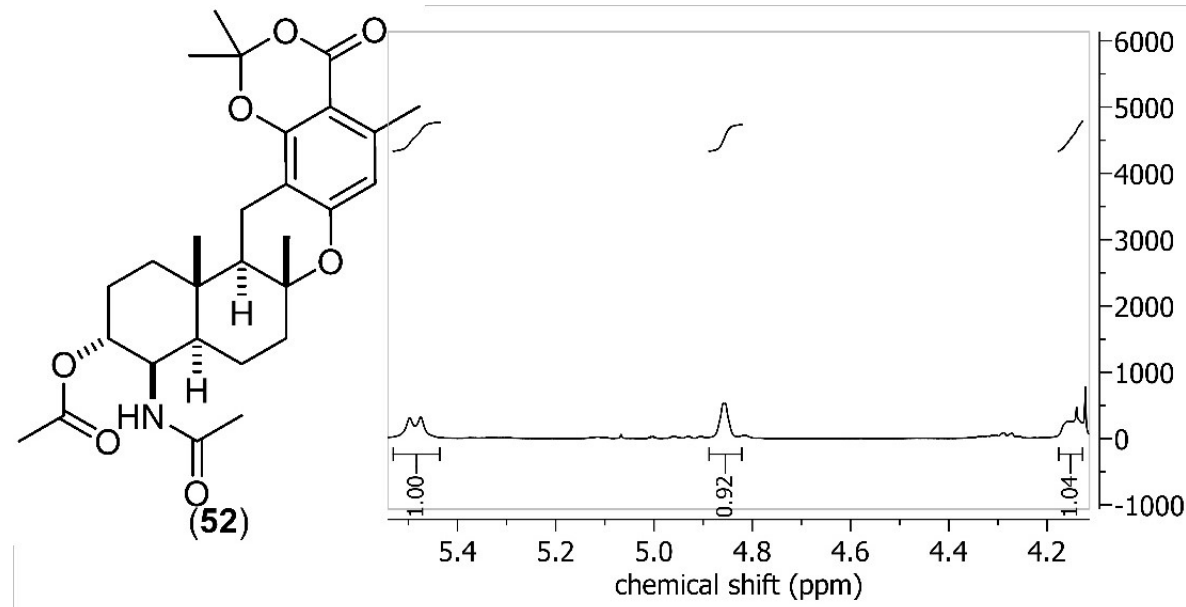

${ }^{1} \mathrm{H}-\mathrm{NMR}\left(400 \mathrm{MHz}, \mathrm{CDCl}_{3}\right) \delta 6.34(\mathrm{~s}, 1 \mathrm{H}), 5.49(\mathrm{~d}, J=9.2 \mathrm{~Hz}, 1 \mathrm{H}, \mathrm{NH}), 4.86(\mathrm{q}, J=$ $2.7 \mathrm{~Hz}, 1 \mathrm{H}), 4.15(\mathrm{dd}, J=10.1,3.9 \mathrm{~Hz}, 1 \mathrm{H}), 2.57(\mathrm{~s}, 3 \mathrm{H}), 2.56(\mathrm{~d}, J=4.6 \mathrm{~Hz}, 1 \mathrm{H}), 2.31$ $-2.20(\mathrm{~m}, 1 \mathrm{H}), 2.14-2.09(\mathrm{~m}, 1 \mathrm{H}), 2.04(\mathrm{~s}, 3 \mathrm{H}), 2.01(\mathrm{~s}, 3 \mathrm{H}), 1.95(\mathrm{~d}, J=11.0 \mathrm{~Hz}$, $1 \mathrm{H}), 1.87-1.84(\mathrm{~m}, 1 \mathrm{H}), 1.83(\mathrm{~d}, J=8.3 \mathrm{~Hz}, 1 \mathrm{H}), 1.78(\mathrm{dd}, J=11.7,6.2 \mathrm{~Hz}, 1 \mathrm{H}), 1.72$ $(\mathrm{s}, 3 \mathrm{H}), 1.69(\mathrm{~d}, J=8.7 \mathrm{~Hz}, 1 \mathrm{H}), 1.67(\mathrm{~s}, 3 \mathrm{H}), 1.66(\mathrm{~s}, 1 \mathrm{H}), 1.58(\mathrm{~d}, J=3.8 \mathrm{~Hz}, 1 \mathrm{H})$, $1.43-1.28(\mathrm{~m}, 1 \mathrm{H}), 1.27$ (d, $J=2.9 \mathrm{~Hz}, 1 \mathrm{H}), 1.21(\mathrm{~s}, 3 \mathrm{H}), 1.01(\mathrm{~s}, 3 \mathrm{H})$.

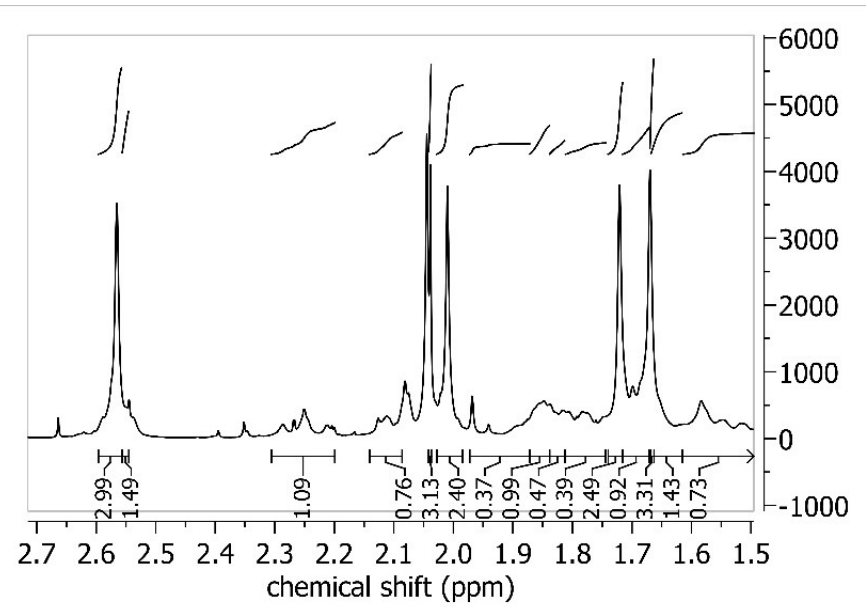

$-8500$

$-8000$

$-7500$

7000

$-6500$

$-6000$

$-5500$

5000

4500

4000

$-3500$

$-3000$

$-2500$

2000

1500

$-1000$

$-500$

$-500$

$\begin{array}{lllllllllll}10.5 & 10.0 & 9.5 & 9.0 & 8.5 & 8.0 & 7.5 & 7.0 & 6.5 & 6.0 & \begin{array}{c}5.5 \\ \text { chemical shift (ppm) }\end{array}\end{array}$

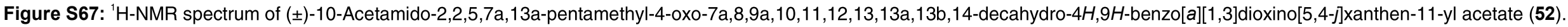




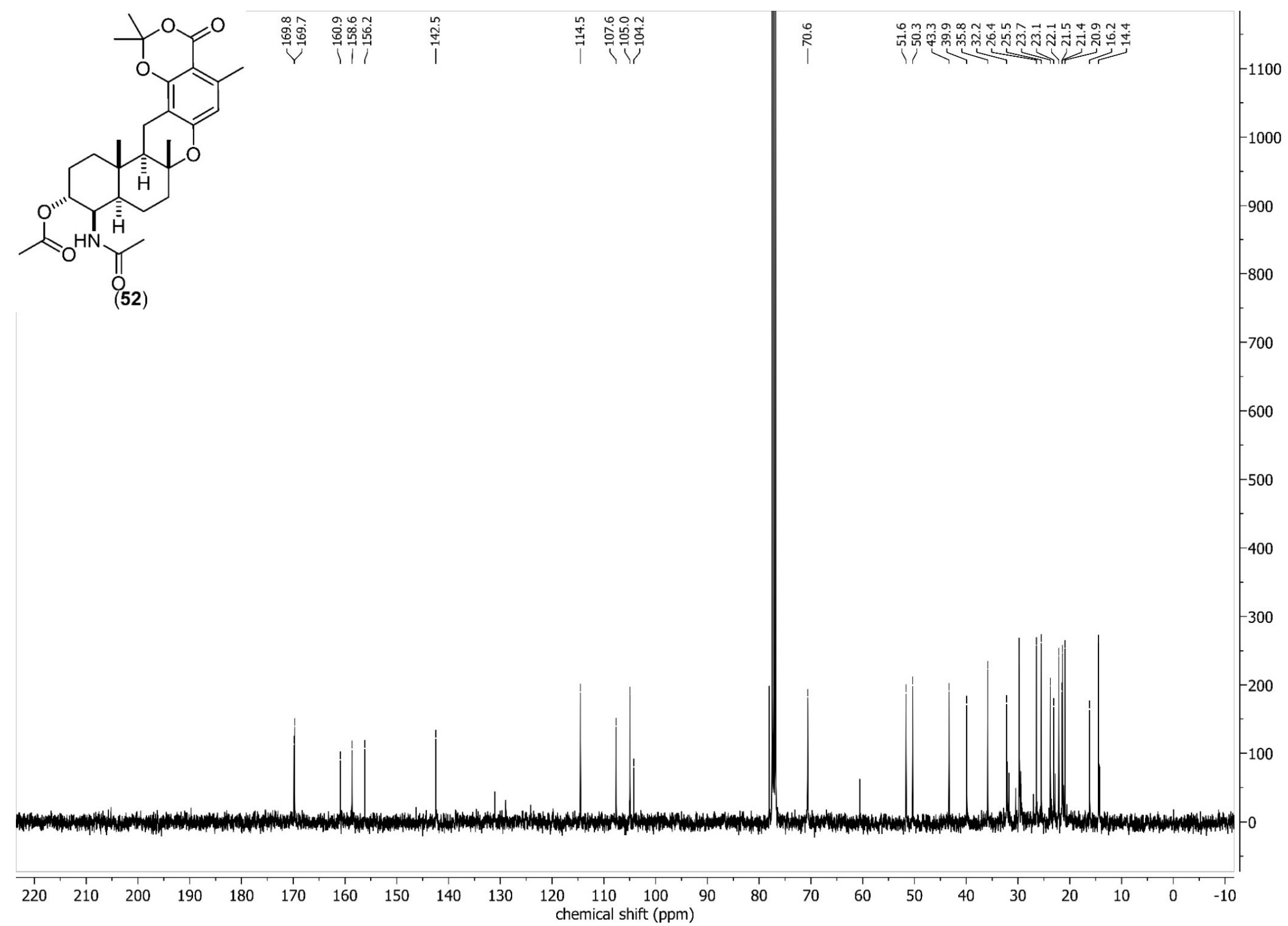

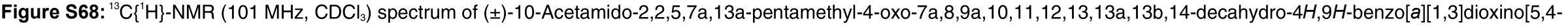
j]xanthen-11-yl acetate (52). 


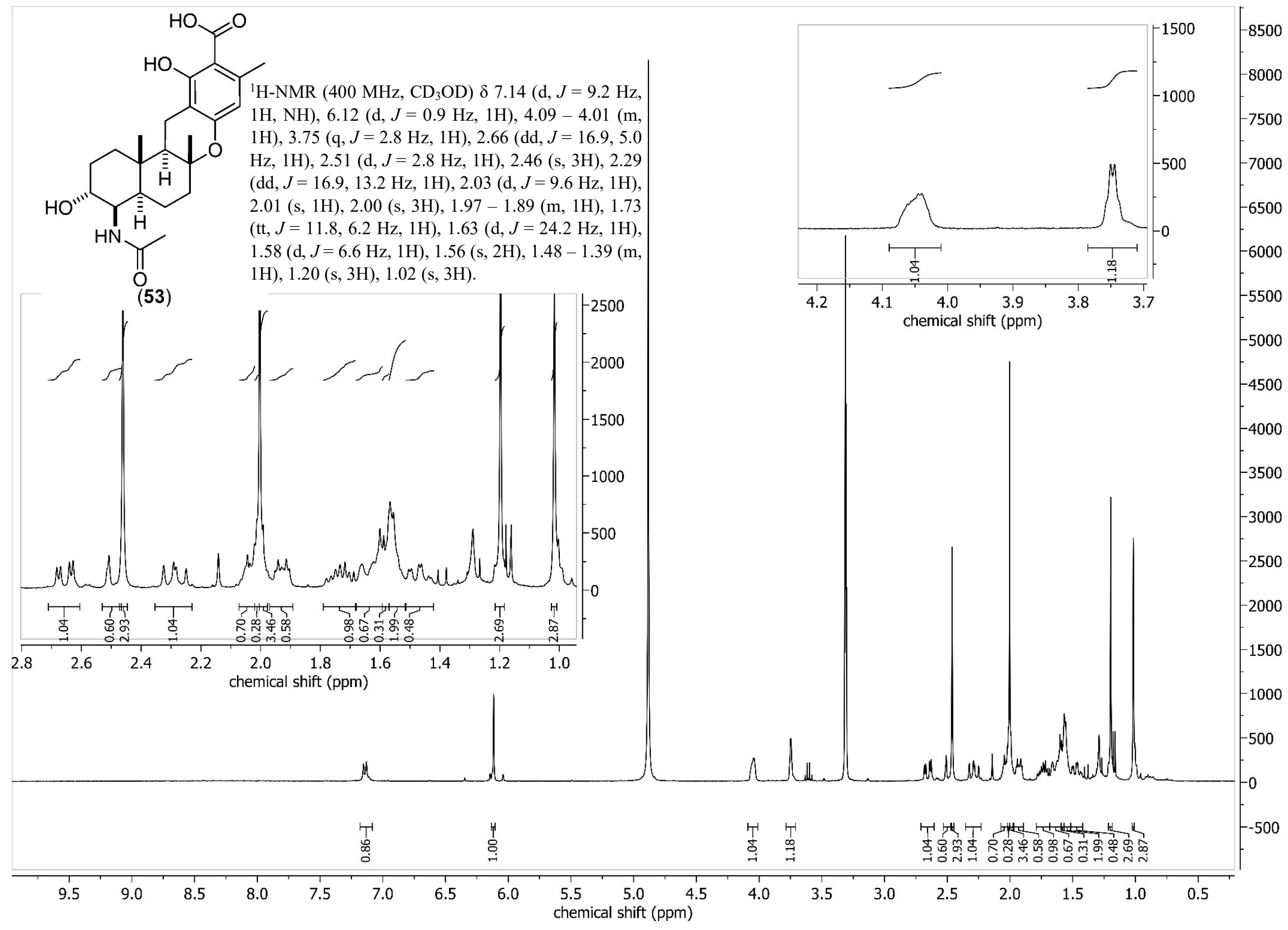

Figure S69: ${ }^{1} \mathrm{H}-\mathrm{NMR}$ spectrum of ( \pm )-4-Acetamido-3,11-dihydroxy-6a,9,12b-trimethyl-1,3,4,4a,5,6,6a,12,12a,12b-decahydro-2H-benzo[a]xanthene-10-carboxylic acid (53). 


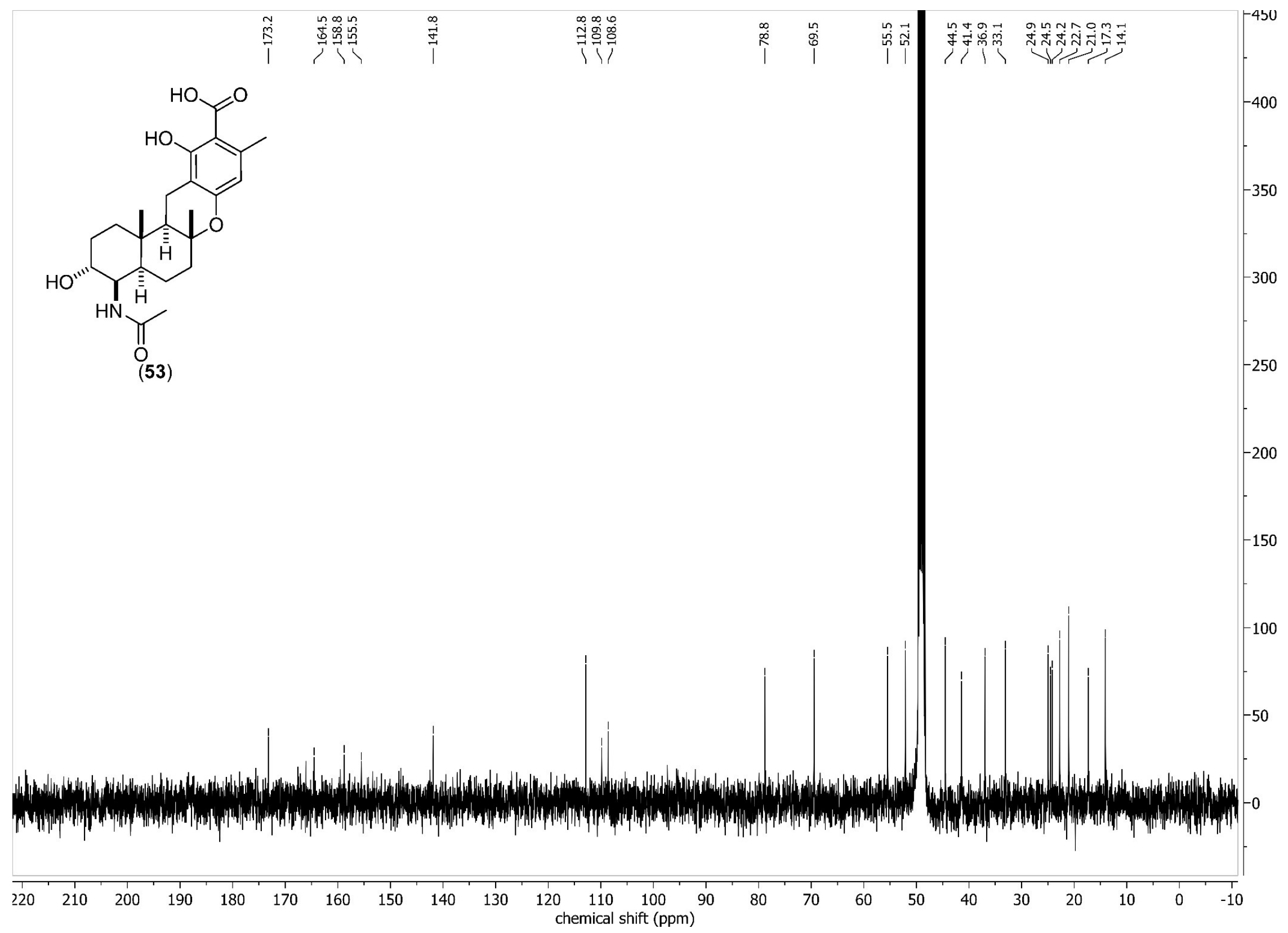

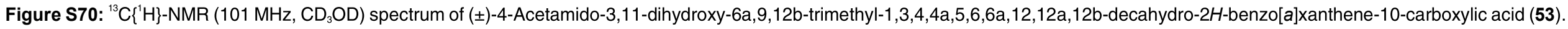




\section{The X-ray Crystal Structure of Hongoquercin Alkene 8}

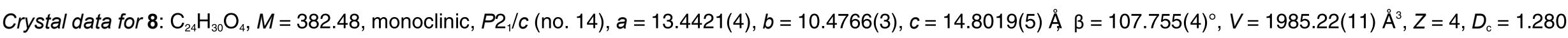

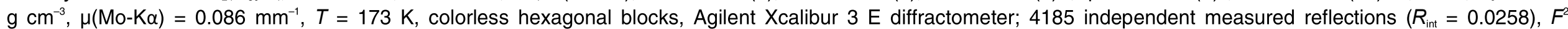

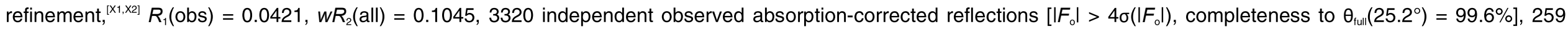
parameters. CCDC 2026869.

\section{The X-ray Crystal Structure of lodo-Alcohol 33}

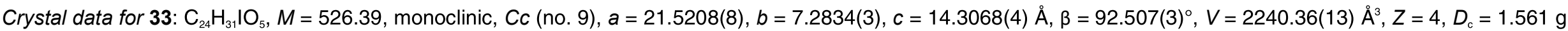

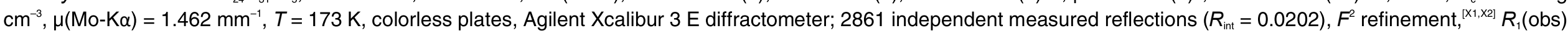

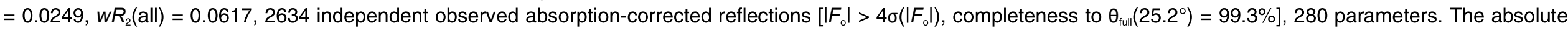
structure of $\mathbf{3 2}$ was determined by use of the Flack parameter $[x=-0.03(2)]$. CCDC 2026870.

The O26-H hydrogen atom in the structure of hydroxy iodide 33 was located from a $\Delta F$ map and refined freely subject to an O- $\mathrm{H}$ distance constraint of $0.90 \AA$

\section{The X-ray Crystal Structure of Bromo-Alcohol 42}

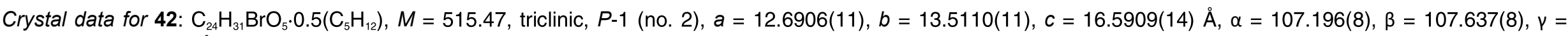

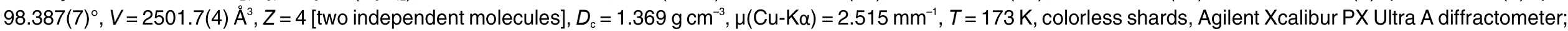

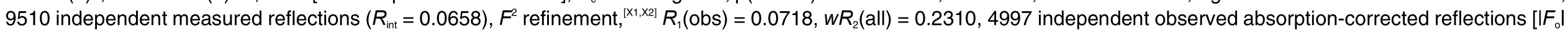
$>4 \sigma\left(\left|F_{\mathrm{o}}\right|\right)$, completeness to $\theta_{\text {full }}\left(67.7^{\circ}\right)=98.0 \%$ ], 635 parameters. CCDC 2026871 .

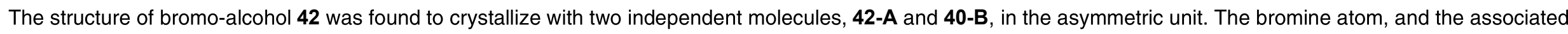

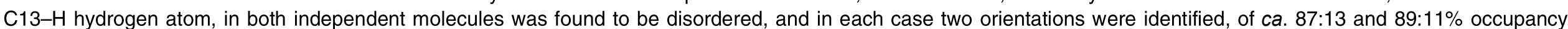

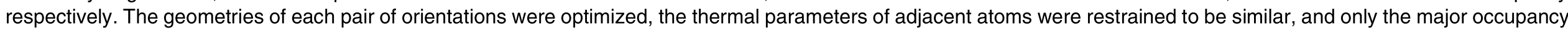

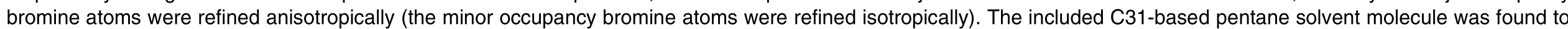

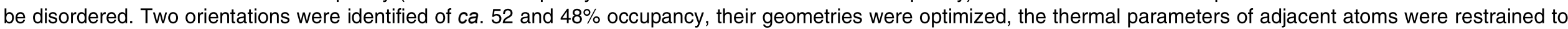

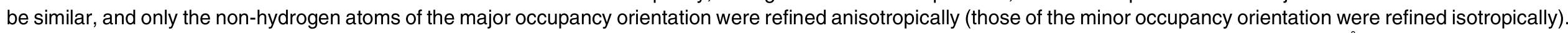
The $\mathrm{O} 26-\mathrm{H}$ hydrogen atom in both independent molecules were located from $\Delta F$ maps and refined freely subject to $\mathrm{O}-\mathrm{H}$ distance constraints of $0.90 \AA$

\section{The X-ray crystal structure of Ketone 45}

Crystal data for 45: $\mathrm{C}_{25} \mathrm{H}_{32} \mathrm{O}_{5}, M=412.50$, orthorhombic, $P 2_{1} 2_{1} 2_{1}$ (no. 19), $a=9.8269(3), b=12.5104(4), c=17.2187(5) \AA, V=2116.85(11) \AA^{3}, Z=4, D_{c}=1.294 \mathrm{~g} \mathrm{~cm}{ }^{-3}$, $\mu(\mathrm{Cu}-\mathrm{K} \alpha)=0.716 \mathrm{~mm}^{-1}, T=173 \mathrm{~K}$, colorless needles, Agilent Xcalibur PX Ultra A diffractometer; 3509 independent measured reflections $\left(R_{\text {int }}=0.0510\right), F^{2}$ refinement, ${ }^{\left[X_{1}, x_{2}\right]}$ $R_{1}($ obs $)=0.0456, w R_{2}($ all $)=0.1182,2823$ independent observed absorption-corrected reflections $\left[\left|F_{\mathrm{o}}\right|>4 \sigma\left(\left|F_{\mathrm{o}}\right|\right)\right.$, completeness to $\left.\theta_{\text {tulu }}\left(67.7^{\circ}\right)=98.7 \%\right], 278$ parameters. The absolute structure of $\mathbf{4 5}$ could not be determined by use of the Flack parameter $[x=0.2(3)]$ and so an arbitrary assignment was made. CCDC 2026872 . 


\section{References}

[X1] SHELXTL v5.1, Bruker AXS, Madison, WI, 1998.

[X2] SHELX-2013; Sheldrick, G.M. Crystal structure refinement with SHELXL. Acta Cryst. 2015, C71, 3-8.

\section{Figures}

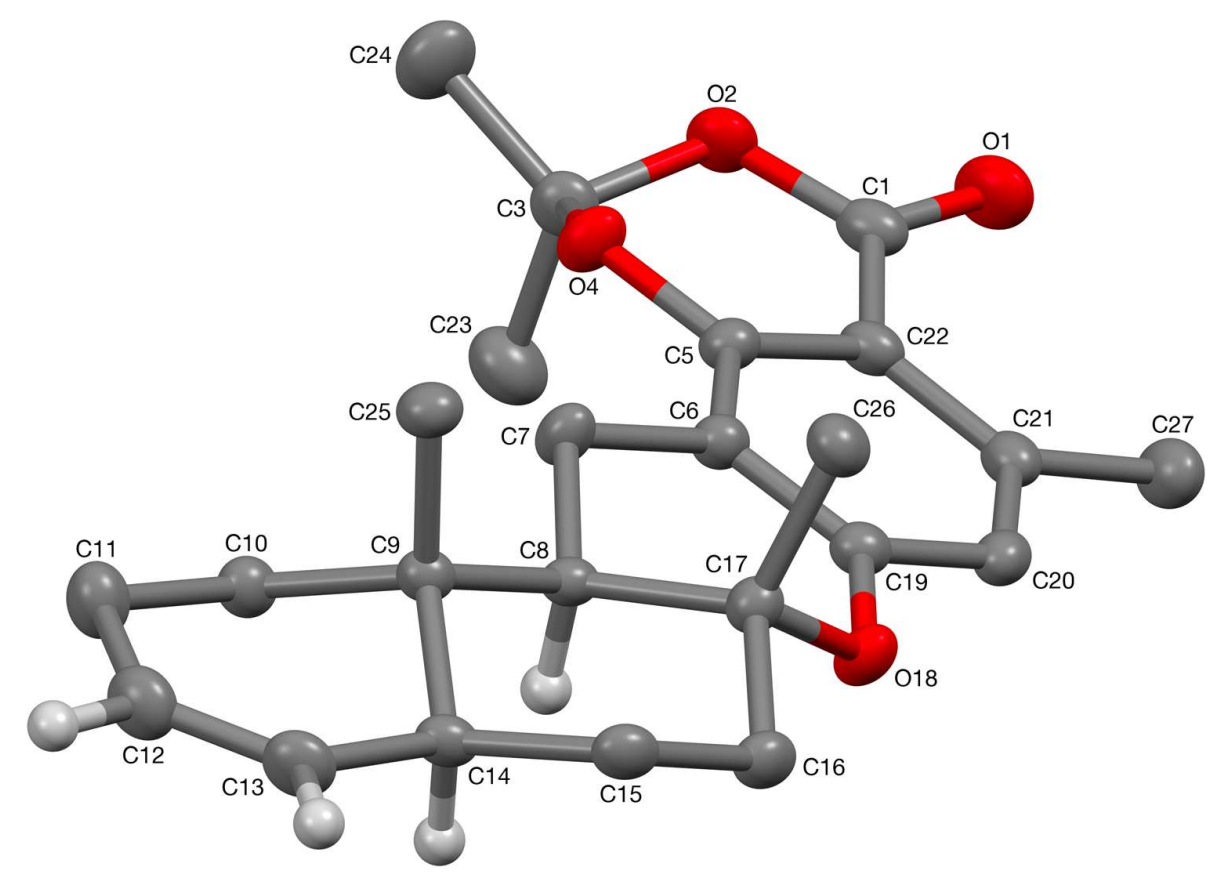

Figure S71: The crystal structure of 8 (50\% probability ellipsoids). 


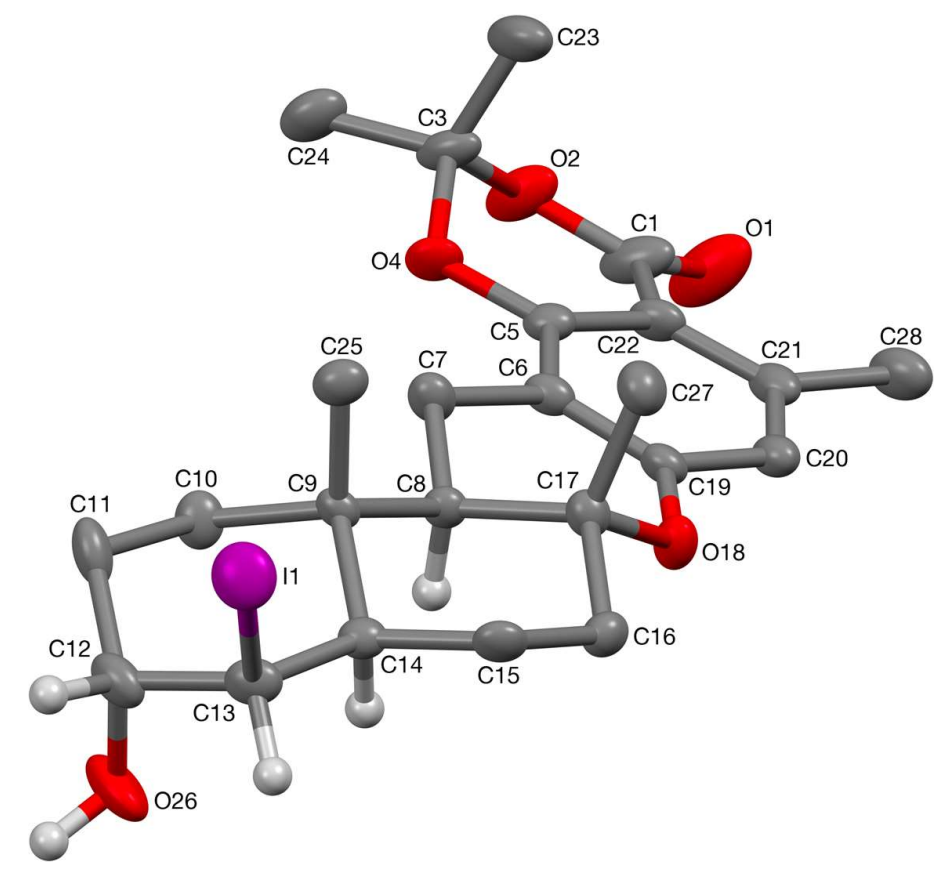

Figure S72: The crystal structure of 33 (50\% probability ellipsoids) 


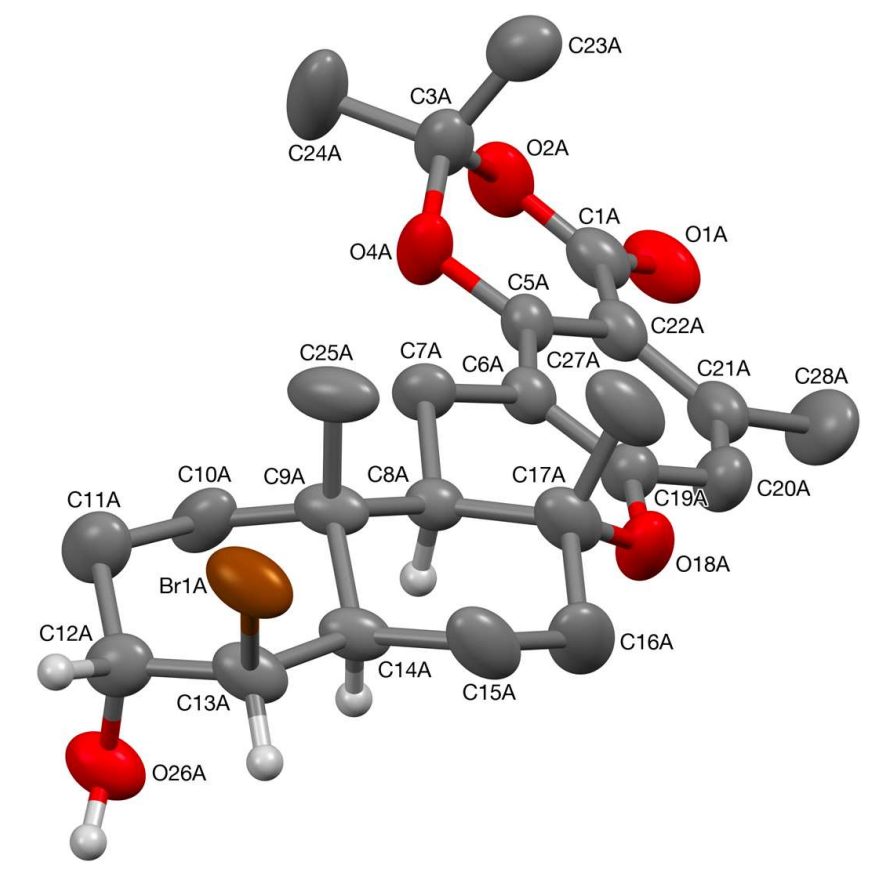

Figure S73: The structure of 42-A, one of the two independent molecules present in the crystal of $\mathbf{4 2}$ (50\% probability ellipsoids). 


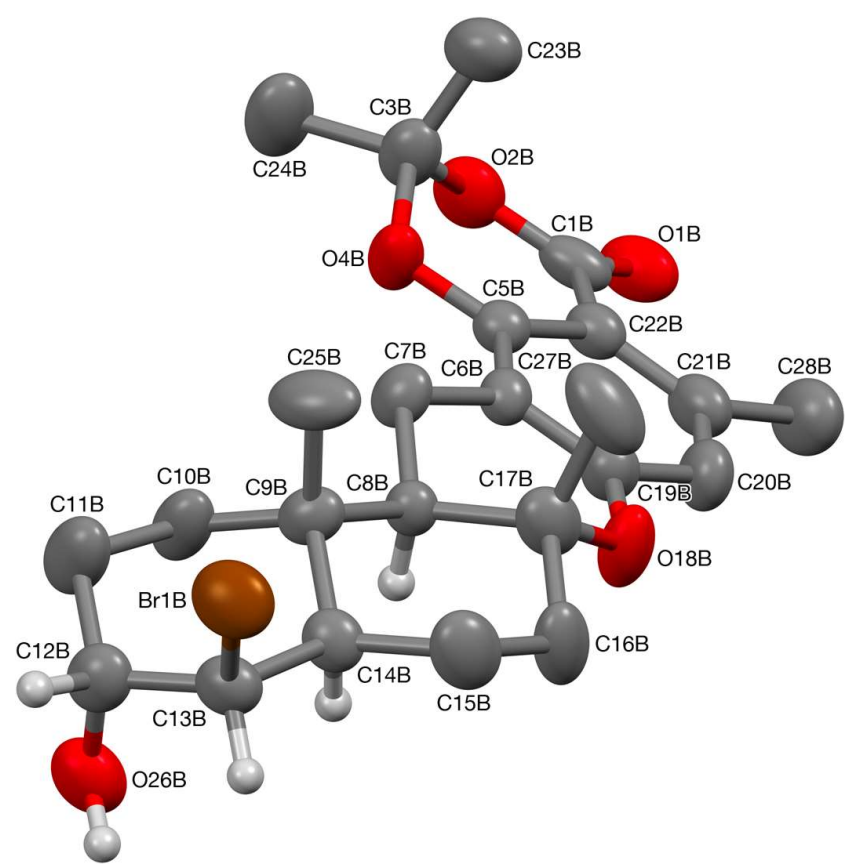

Figure S74: The structure of 42-B, one of the two independent molecules present in the crystal of $\mathbf{4 2}$ (50\% probability ellipsoids). 


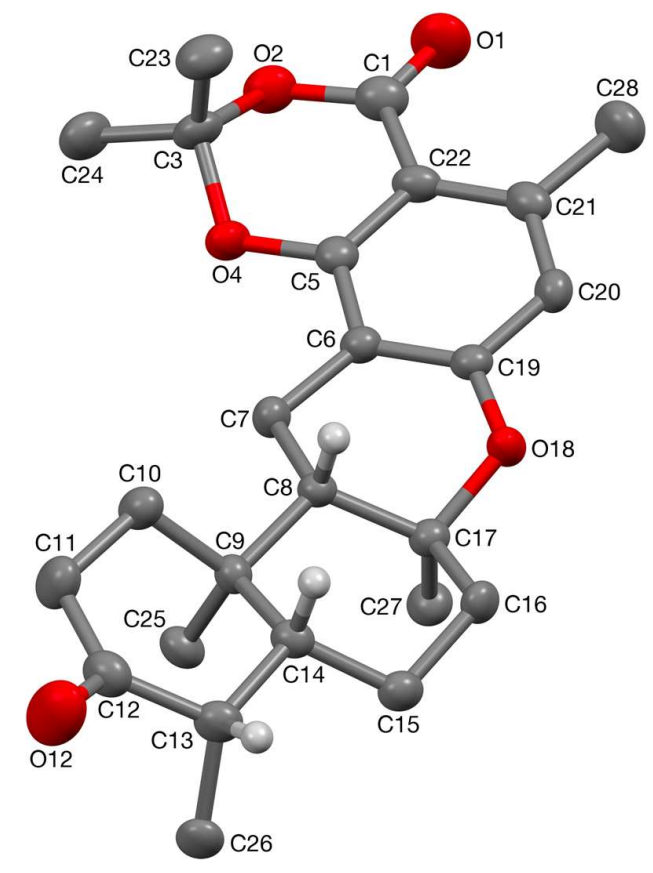

Figure S75: The crystal structure of $\mathbf{4 5}$ (50\% probability ellipsoids). 